\title{
Novel approach for identification of biocatalysts by reverse omics techniques
}

\author{
Dissertation \\ for the award of the degree \\ "Doctor rerum naturalium" \\ of the Georg-August-Universität Göttingen
}

within the doctoral program "Microbiology and Biochemistry"

of the Göttingen Graduate School for Neurosciences, Biophysics, and Molecular Biosciences (GGNB)

submitted by

Richard Egelkamp

from Rheine 


\section{Thesis Committee}

Prof. Dr. Rolf Daniel, Genomic and Applied Microbiology,

Institute for Microbiology and Genetics, Georg-August-Universität Göttingen

PD Dr. Michael Hoppert, General Microbiology,

Institute for Microbiology and Genetics, Georg-August-Universität Göttingen

Prof. Dr. Jörg Stülke, General Microbiology,

Institute for Microbiology and Genetics, Georg-August-Universität Göttingen

\section{Members of the Examination Board}

Referee: Prof. Dr. Rolf Daniel, Genomic and Applied Microbiology,

Institute for Microbiology and Genetics, Georg-August-Universität Göttingen

2nd referee: PD Dr. Michael Hoppert, General Microbiology,

Institute for Microbiology and Genetics, Georg-August-Universität Göttingen

\section{Further members of the Examination Board}

Prof. Dr. Jörg Stülke, General Microbiology,

Institute for Microbiology and Genetics, Georg-August-Universität Göttingen

Prof. Dr. Gerhard Braus, Molecular Microbiology and Genetics,

Institute for Microbiology and Genetics, Georg-August-Universität Göttingen

Prof. Dr. Kai Heimel, Molecular Microbiology and Genetics,

Institute for Microbiology and Genetics, Georg-August-Universität Göttingen

Prof. Dr. Stefanie Pöggeler, Genetics of Eukaryotic Microorganisms,

Institute for Microbiology and Genetics, Georg-August-Universität Göttingen

Date of oral examination: 20.02 .2019 


\section{Table of content}

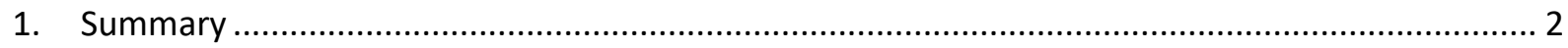

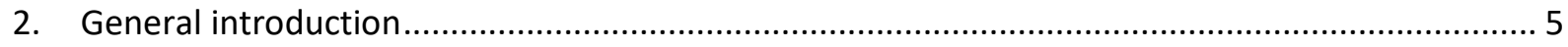

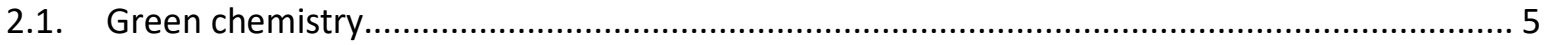

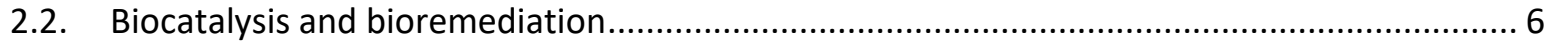

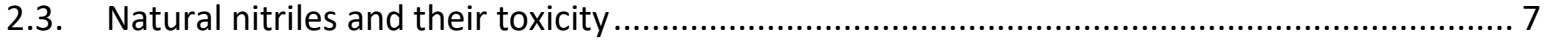

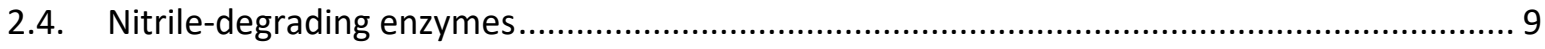

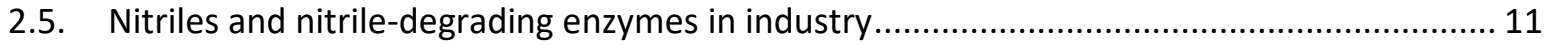

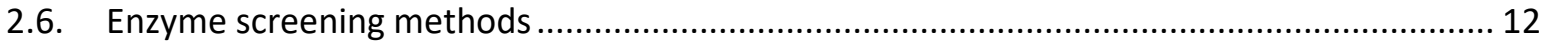

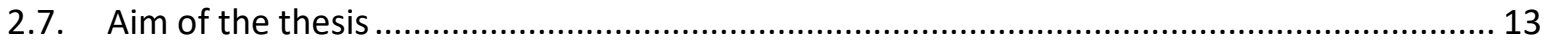

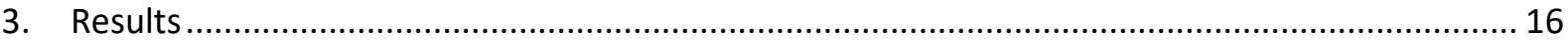

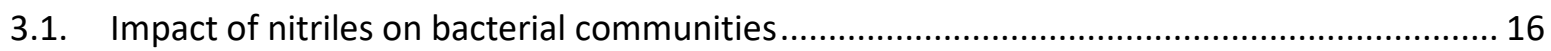

3.2. Nitrile-degrading bacteria isolated from compost ............................................................. 42

3.3. From sequence to function: A new workflow for nitrilase identification.............................. 51

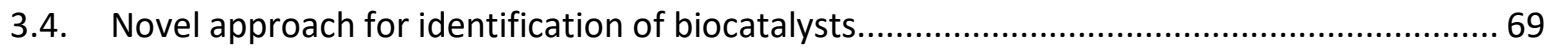

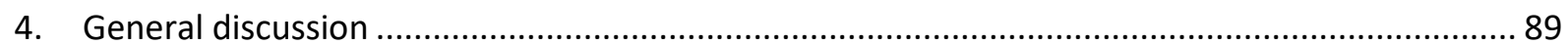

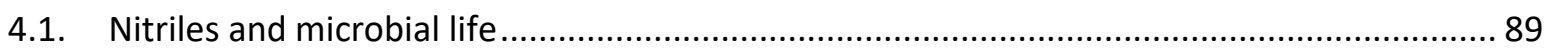

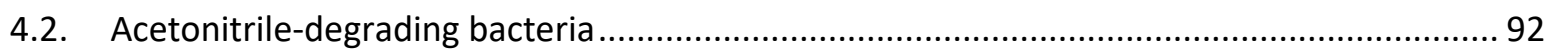

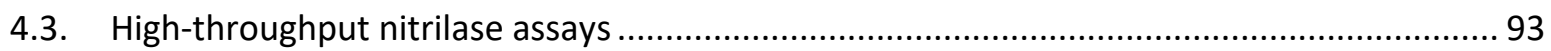

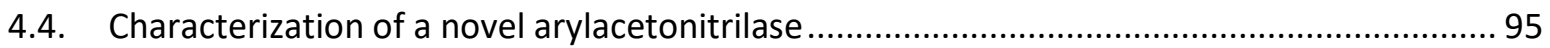

4.5. A novel (meta)transcriptomic screening approach ............................................................ 96

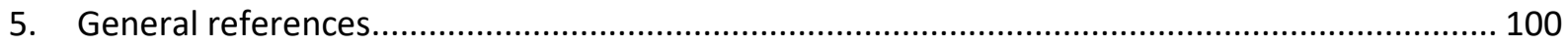

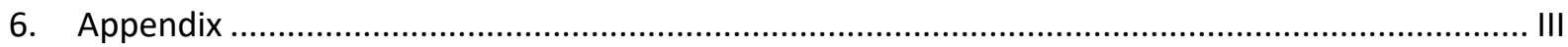

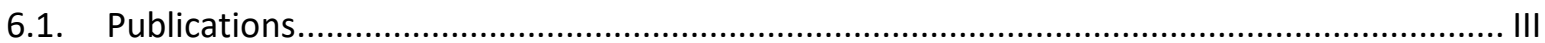

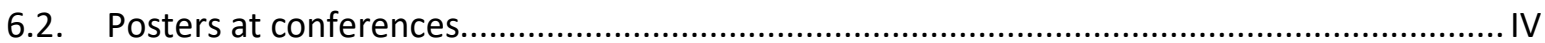

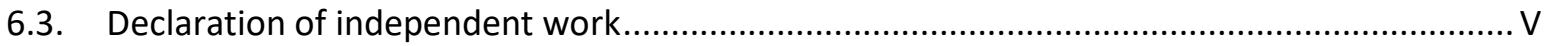

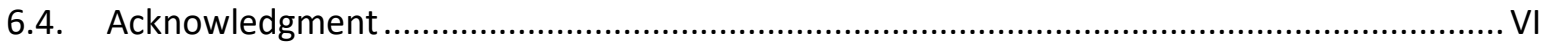


Chapter I

Summary 


\section{Summary}

A novel screening approach for identification of biocatalysts by reverse omics techniques was developed. Basic principle is the differential analysis of metatranscriptomes obtained from cultures treated either with the substrate or the product of the enzymatic reaction of interest. All genes upregulated in the substrate-containing culture respond to the added compound and could be involved in its degradation or belong to respective transporters or resistance mechanisms.

Nitrilases, enzymes degrading often toxic nitriles to the corresponding carboxylic acids and ammonia, were chosen as biocatalysts of interest. As effect of nitriles on microbial life is barely known, their influence had to be determined before establishment of the screening approach. For that purpose, agar plates containing different concentrations of nine tested nitriles (phenylacetonitrile, succinonitrile, acetonitrile, crotononitrile, 4-hydroxybenzonitrile, acetone cyanohydrin, cyclohexanecarbonitrile, 2-phenylpropionitrile, and pyruvonitrile) were prepared. Growth of Agrobacterium tumefaciens, Bacillus subtilis, Corynebacterium glutamicum, and Escherichia coli was monitored on these plates and revealed first insights into nitrile toxicity. Subsequently, highest nontoxic concentrations were used to treat liquid cultures containing a microbial community derived from compost. Growth was monitored regularly and cultures were transferred to fresh medium every second day to avoid nutrient depletion. Acetone cyanohydrin, 2-phenylpropionitrile, and pyruvonitrile exhibited a lethal effect on the microbial community. In contrast, cultures containing succinonitrile, acetonitrile, and crotononitrile showed higher optical densities than the control, indicating a growthsupporting effect. Furthermore, community composition was determined by $16 \mathrm{~S}$ rRNA gene analysis and metagenome sequencing, revealing specific community-shaping effects for every compound, i.e. Pseudomonas was detected in most cultures whereas Paenibacillus was highly abundant in cultures containing growth-suppressing nitriles. In general, Gram-positive bacteria showed higher nitrile tolerance than Gram-negative bacteria.

Growth-supporting effect of acetonitrile during nitrile toxicity analysis indicated metabolization of this compound and therefore presence of nitrile-degrading enzymes. To increase knowledge on nitriledegrading organisms and obtain strains for establishment of the screening approach, isolation of respective organisms was performed. Finally, eight different isolates belonging to Flavobacterium, Pseudomonas, Rhodococcus, and Variovorax were identified. Nitrile-degradation by the latter three is common, but only weak degradation by Flavobacterium has been reported before. Genome sequencing of the isolates revealed various nitrile-degrading enzymes for all strains except Flavobacterium, indicating a novel mechanism for nitrile degradation in this genus.

Analysis of metagenomes obtained during nitrile toxicity test revealed 70 putative nitrilases in nitriletreated cultures. Due to their origin, they were promising candidates for identification of novel nitrile- 
degrading biocatalysts. For fast and simple screening of this number of enzymes, a novel highthroughput assay was developed. The new method combines real-time measurement of enzymatic activity and high sensitivity without dependency on purified proteins. Six putative enzymes exhibited nitrilase activity and subsequently, the most interesting biocatalysts was further characterized. The novel nitrilase exhibits a broad pH optimum and unusually high long-term stability. Furthermore, it is highly specific for phenylacetonitrile and belongs to the class of arylacetonitrilases, which are of industrial importance but rarely studied.

Finally, the proposed screening approach for identification of biocatalysts by reverse omics techniques was developed. For initial tests, the previously isolated acetonitrile-degrading Rhodococcus was used. Growth condition, cell harvesting, and isolation of DNA and RNA were optimized with this strain. Subsequently, a protocol for normalization and differential analysis of transcriptomes was established. Only one operon consisting of three genes and a nearby amidase were significantly upregulated in the nitrile-treated cultures. The operon did not encode for known nitrile-degrading proteins, but proximity to the amidase supports the respective activity. Subsequently, the established parameters were applied to a complex microbial community. Metatranscriptomes were normalized based on the abundance of single species to allow statistically valid analyses. More than 500 up- and 280 downregulated genes could be identified under nitrile-treatment, indicating complex interactions between members of the community. For one species, a highly upregulated nitrilase and amidase were found, whereas no significant upregulation of respective genes was recorded for other species. Therefore, unknown nitrile-degrading enzymes may hide behind upregulated putative proteins, demonstrating the potential of this novel metatranscriptomic screening approach. 
Chapter II

General introduction 


\section{General introduction}

During the first half of the $19^{\text {th }}$ century, the era of modern chemistry began. In 1835 , Jöns Jacob Berzelius first mentioned "catalysis" as a process in which a compound influences a chemical reaction without being part of the substrates or products (1). Together with observations of the catalytic reactivity of metals like platinum, palladium, and iridium $(2,3)$, a new research field of chemistry was created. Early industrial application of catalysts started in 1913 with the synthesis of up to 30 tons ammonia per day using an iron catalyst (4). In the following decades, numerous other catalytic processes were established, e.g. the synthesis of hydrocarbons using carbon monoxide and hydrogen, the synthesis of methanol, the polymerization of ethylene to polyethylene, and cracking of petroleum using zeolite as catalyst (5).

Even though modern civilization would not be possible without chemical industry, large scale production results in a severe environmental footprint. Many chemical processes need strong acids or bases, (heavy) metal catalysts, high reaction temperature, and produce large amounts of inorganic waste. In addition, the processes can yield unwanted isomers (6-8). For example, the production of 1 ton ammonia leads to the release of 1.5 tons carbon dioxide. In addition, global ammonia production is responsible for $2 \%$ of the total industrial energy consumption (9). Subsequently, ammonia can be used for production of compounds like acrylonitrile. More than 7 million tons of this important monomer are produced per year (10), but synthesis depends on high temperature and pressure. In addition, several purification steps for removal of contaminants like hydrogen cyanide or acetone are necessary to yield pure acrylonitrile (11).

With a growing population and increasing standards in developing countries, limited resources and environmental problems like climate change become more challenging. Therefore, chemical industry must advance and develop new environmentally friendly processes to fulfill the growing demand for bulk and fine chemicals.

\subsection{Green chemistry}

During the 1980s, waste production and use of toxic or hazardous materials in industry became a growing concern (12), finally leading to the U.S. Pollution Prevention Act of 1990 (13). In contrast to previous governmental guidelines it focused on pollution prevention rather than waste treatment, marking the beginning of the green chemistry era. Subsequently, twelve principles of green chemistry were developed (14):

1) Waste should be prevented instead of being treated

2) Incorporation of all involved materials into the final product should be maximized 
3) Use or generation of toxic substances during production should be minimized

4) Toxicity of final products should be reduced

5) Use of auxiliary substances like solvents should be avoided

6) Energy requirements of chemical processes should be minimized

7) Renewable feedstock or raw material should be used when practical

8) Unnecessary derivatization should be minimized

9) Catalytic reagents should be preferred over stoichiometric reagents

10) Products should break down into innocuous products at the end of their use

11) Real-time analyses should be further developed to prevent pollutant formation

12) Inherently safer chemicals should be used for accident prevention

These principles are nowadays often used as guideline for the development of new production lines. The most promising approach for a sustainable green chemistry is the transition to biobased processes, as demonstrated by novel processes for production of acrylonitrile. Traditional synthesis is based on ammonia and propene, a byproduct of oil refining, and therefore not sustainable. In contrast, newly developed synthesis routes use renewable substrates like glycerol or glutamic acid (15). Furthermore, they can achieve higher yields and avoid by-products like hydrogen cyanide (10). Nevertheless, not all aims of green chemistry can be fulfilled by change of substrates, as these novel processes still need unfavorable conditions and metal catalysts. Consequently, enzymes as biocatalysts are of increasing interest as they can help to meet 10 of the 12 criteria of green chemistry (16).

\subsection{Biocatalysis and bioremediation}

Although the principles of catalysis were almost unknown, the potential of enzymes was already discovered in the 19th century, when Louis Pasteur demonstrated the conversion of L-(+)-tartaric acid to D-(-)-tartaric acid using Penicillium glaucum (17). In 1874, a Danish company (Christian Hansen's Laboratory) started selling enzyme preparations for the production of cheese (18). In 1897, Eduard Buchner showed that living organisms are unnecessary for enzymatic activity by using cell-free yeast extract for fermentation of sugar, further increasing the interest on biocatalysts (19).

Enzymes received attention by biologists, but chemists did not recognize their potential as (bio)catalysts (20). Even the immobilization of enzymes and a similar handling to classic chemical catalysts did not change this view (21). One of the main reasons for this difference may have come from limitations during enzyme isolation. As natural producers were often difficult to grow, only small isolation volumes and therefore irrelevant quantities for industrial purposes were obtained. This problem was solved with development of recombinant DNA techniques in the 1970s and PCR in 1983. Transfer of interesting genes into heterologous easy-to-handle host organisms like Escherichia coli or 
Bacillus subtilis suddenly allowed the efficient production of enzymes (22). In addition, development of directed mutagenesis and directed evolution allowed enhancements of substrate range or protein stability, finally leading to industrially relevant enzymes $(20,23,24)$.

Modern biocatalysts show high selectivity, work under mild conditions, and can be used without the production of critical waste (25). Especially the pharmaceutical industry has interest in biocatalysts as many conventional drug synthesis processes produce large amounts of waste (26). The early use of a lyase for the production of L-3,4-Dihydroxyphenylalanin, an important drug for the treatment of Parkinson's disease, underlines this interest (27). Nowadays, application of biocatalysts is widespread. The food industry uses enzymes for bread and cheese production (28), whereas bulk production of bioethanol from starch ( 42 million $\mathrm{m}^{3}$ per year) demonstrates the full potential of enzymatic processes (21). The huge industrial interest reflects also on market data: In 2018 the global enzyme market had a value of US $\$ 5.5$ billion, but is expected to reach US $\$ 7.0$ billion in 2023 (29).

With rising awareness of environmental pollution in the public, waste prevention and remediation became growing industrial sectors. Bioremediation aims to remove pollution appearing during production processes or remediate already contaminated environments by using (genetically modified) microorganisms. Examples are the biosorption of metals like nickel, lead, or cadmium from soil or wastewater $(30,31)$ or the bioremediation of radionucleotide-contaminated sites $(32,33)$. Furthermore, microorganisms can be used for removal of mutagenic or carcinogenic dyes from textile industry wastewater $(34,35)$. In rare cases, it can even be the only option for environmental decontamination. During an earthquake in India in 2001, damaged acrylonitrile tanks led to the release of the chemical and contamination of surrounding soil. Eight months after leakage, no reduction of nitrile concentration could be measured, demonstrating its persistence. Subsequent bioaugmentation and nutrient-supplementation helped to establish microbial soil communities with nitrile-degrading properties, finally leading to successful decontamination of the environment $(36,37)$.

\subsection{Natural nitriles and their toxicity}

Nitriles are toxic compounds with $-\mathrm{C} \equiv \mathrm{N}$ as functional group. Most nitriles are synthesized from amino acids (tyrosine, phenylalanine, valine, isoleucine, and leucine) which are converted to aldoximes and subsequently transformed to nitriles or cyanogenic glycosides (Figure 1) (38-40). Nitriles are widespread in nature and have been identified in form of hydrogen cyanide in insects $(41,42)$, as aeroplysinin in sponges (43), or as antibiotics like borrelidin or toyocamycin in bacteria $(44,45)$. Furthermore, several nitrile-containing compounds have been reported for fungi (46-48). The most prominent group of nitriles are cyanogenic glycosides, which can be found in over 3,000 plant species belonging to more than 130 families (49). 
Most of the naturally occurring nitriles and cyanides seem to be involved in defense mechanisms. Toyocamycin as nucleoside antibiotic interferes with cell wall synthesis (45) and borrelidin inhibits the activity of threonyl-tRNA synthetases (50). For aeroplysinin, several protective functions like antibiotic, anti-inflammatory, or anti-angiogenic effects have been described (51). Main purpose of cyanogenic glycosides seems to be defense against predators by degradation to hydrogen cyanide after cell disruption or attack $(52,53)$, but use as nitrogen storage has also been described $(54)$.

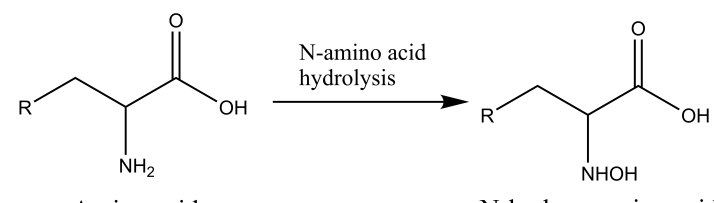

Amino acid

N-hydroxyamino acid

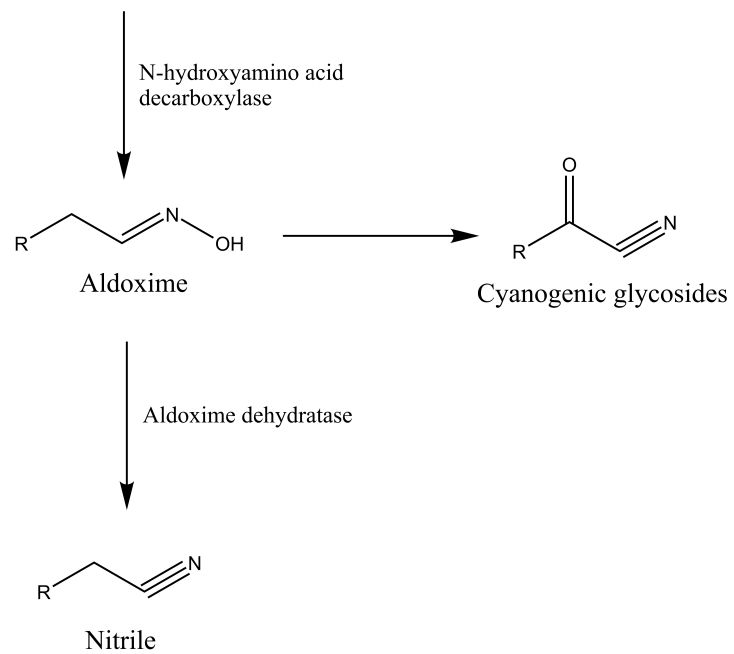

Figure 1: Nitrile synthesis in nature. Adapted from Prasad et al., 2010 (38).

Although many nitriles have toxic effects, toxicity does not derive from the nitrile structure itself. The proposed mechanism in eukaryotes assumes nitrile degradation by cytochrome P450 to an unstable cyanohydrin. Spontaneous decomposition of this compound leads to the release of highly toxic cyanide, which can inhibit cytochrome c oxidases (55-59). As this enzyme is the terminal electron acceptor of the respiratory chain, inhibition leads to asphyxiation and can be lethal. Even though respective studies for bacteria are missing, a similar mode of action can be assumed as cytochrome P450 and cytochrome c oxidase are present in many bacteria $(60,61)$.

Besides cyanide release, other mechanisms for toxicity are also known. For example, main toxicity of acrylonitrile arises from interaction with thiol groups, affecting central and peripheral nervous systems (62). As thiol groups are also important for activity of numerous bacterial enzymes, similar toxicity mechanisms may apply. Other mechanisms may also account for toxicity of chloroacetonitrile, isovaleronitrile, caprylonitrile, and benzonitrile, as no linkage between nitrile concentration and intracellular cyanide presence could be found (55). In addition, chemical structure seems to have an influence on toxicity. An increased chain length appears to reduce toxicity and dinitriles are slightly 
less toxic than mononitriles. Nevertheless, toxicity values observed for the first nitrile of a homologous series cannot be extrapolated to other members of such a group (63).

\subsection{Nitrile-degrading enzymes}

As nitriles and cyanides are toxic to living organisms, different enzymatic detoxification strategies exist (Figure 2). For degradation of cyanide, mainly two pathways are used. The first involves cyanide hydratases (EC 4.2.1.66) catalyzing the degradation of cyanide to formamide. They are most likely exclusively fungal enzymes consisting of several subunits and can reach up to $3,000 \mathrm{kDa}(64-66)$. The second pathway uses cyanide dihydratases that degrade cyanide to formic acid and ammonia. So far, this reaction was only detected in a few bacterial genera (67). Like cyanide hydratases, cyanide dihydratases do not require metal cofactors and are therefore interesting candidates for bioremediation of cyanide-contaminated sites (68). In addition to these two options, other pathways such as direct degradation to carbon dioxide and ammonia by cyanide dioxygenases or to methane and ammonia by nitrogenases are known (69).

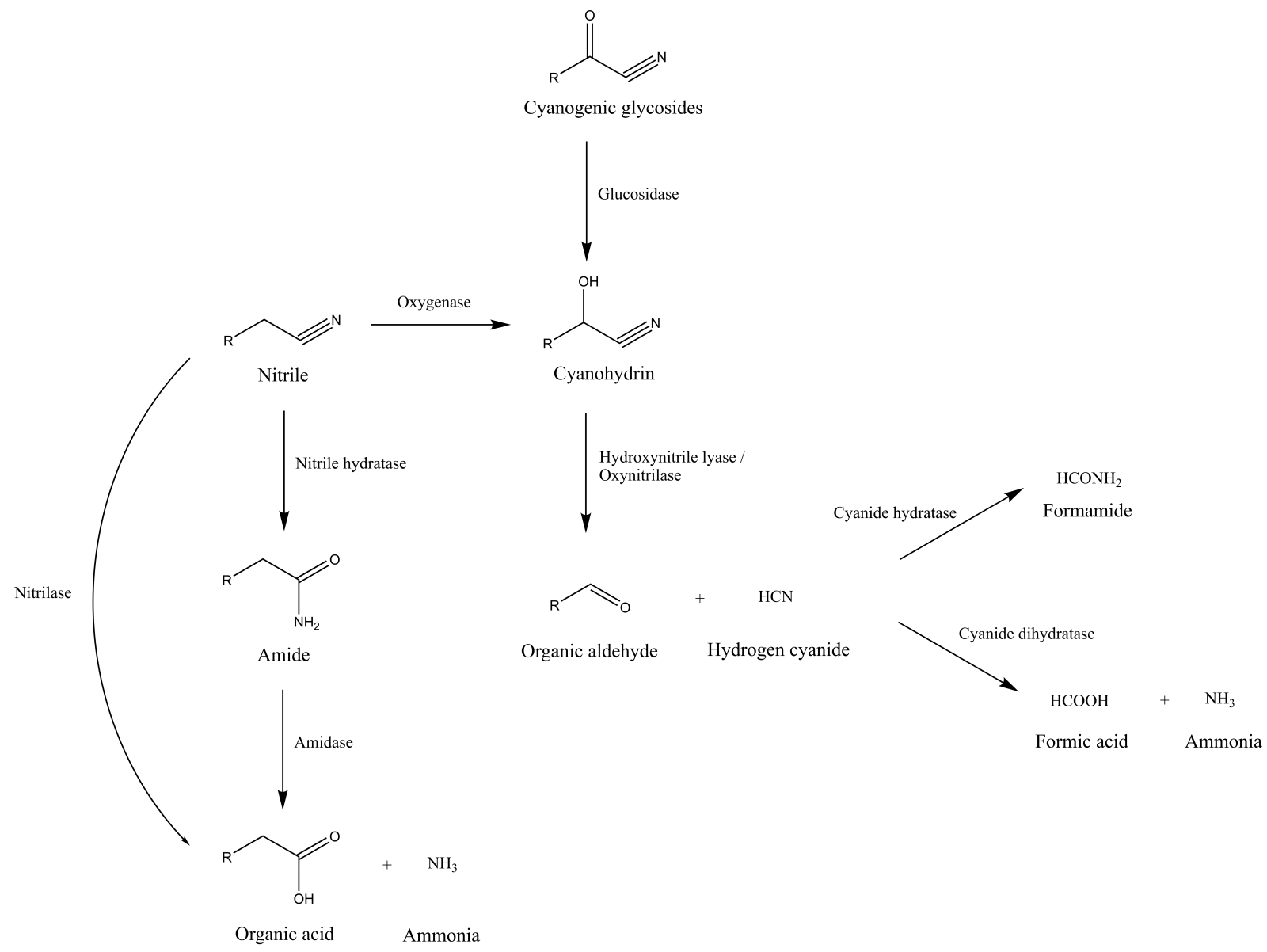

Figure 2: Enzymatic nitrile degradation. Adapted from Prasad et al., 2010 (38). 
Degradation of nitriles is mainly performed by two pathways. The first involves nitrilases (EC 3.5.5.1), which degrade nitriles directly to carboxylic acid and ammonia. These enzymes are known in archaea, bacteria, fungi, plants, and animals (70) and belong to the carbon-nitrogen hydrolase superfamily. Nitrilases are classified according to their preferred substrates into aliphatic, aromatic, and arylacetonitrilases (71). They consist of $6-26$ subunits with an $\alpha-\beta-\beta-\alpha$ fold and an average mass of $40 \mathrm{kDa}$ (72-74). Important for enzymatic activity is a metal-independent conserved catalytic triad which is present in all known members of the nitrilase family and consists of glutamic acid, lysine, and cysteine $(72,75-78)$. Majority of these enzymes have a temperature optimum of activity between $30{ }^{\circ} \mathrm{C}$ and $55^{\circ} \mathrm{C}$ and show catalytic activity under neutral or slightly alkaline conditions (74). Their expression is normally induced by their substrates or products, but a few cases of constitutive nitrilase expression have also been described (79-81).

The second pathway for nitrile degradation consists of two enzymes, nitrile hydratases (NHases; EC 4.2.1.84) and amidases (EC 3.5.1.4). The former degrade nitriles to amides and the latter subsequently the amides to carboxylic acid and ammonia (82). In contrast to nitrilases, NHases belong to the carbon-oxygen lyase superfamily and are found in several bacterial genera and few eukaryotes $(38,83)$. They consist of an $\alpha$ - and $\beta$-subunit with average molecular masses of around $25 \mathrm{kDa}$ $(38,84,85)$. Based on the metal cofactor bound to their $\alpha$-subunit, these enzymes are often divided into cobalt or ferric NHases (86). Besides the cofactor, conserved amino acids seem to be important for catalytic activity. For cobalt NHases, respective threonine and tyrosine residues have been identified, whereas specific serine and threonine residues are conserved in ferric NHases $(87,88)$. In addition, it has been shown that at least for a NHase from Streptomyces rimosus the $\alpha$-subunit is sufficient for enzymatic activity (89). In contrast to nitrilases, NHases are often thermolabile and exhibit highest activity between $30^{\circ} \mathrm{C}$ and $40^{\circ} \mathrm{C}$, but share the same optimal pH range at around $\mathrm{pH} 7$ (38).

Amidases, the second enzyme of this bi-enzymatic pathways, are similar to nitrilases. They also belong to the carbon-nitrogen hydrolase superfamily and contain the same conserved catalytic triad (90). These ubiquitous enzymes have an average molecular mass of around $40 \mathrm{kDa}$ and can consist of monomers, dimers, tetramers, hexamers, or octamers (91-94). Except for amidases involved in cell separation using zinc, cofactors are not required for enzymatic activity (95). Most of the characterized amidases show optimum activity at neutral or slightly alkaline $\mathrm{pH}$ values and temperatures between 40 and $55^{\circ} \mathrm{C}(91,96-99)$. 


\subsection{Nitriles and nitrile-degrading enzymes in industry}

Nitriles as well as nitrile-derived amides and carboxylic acids are of great importance for industry. The first amidase was described in 1949 (100), followed by nitrilases in 1964 (101), and NHases in 1980 (102). Increasing numbers of publications for all these enzymes demonstrate the intensified research activity in this area (Figure 3). Nevertheless, literature on nitrile-degrading enzymes is scarce when compared to other enzymes like phosphatases, esterases, or lipases.

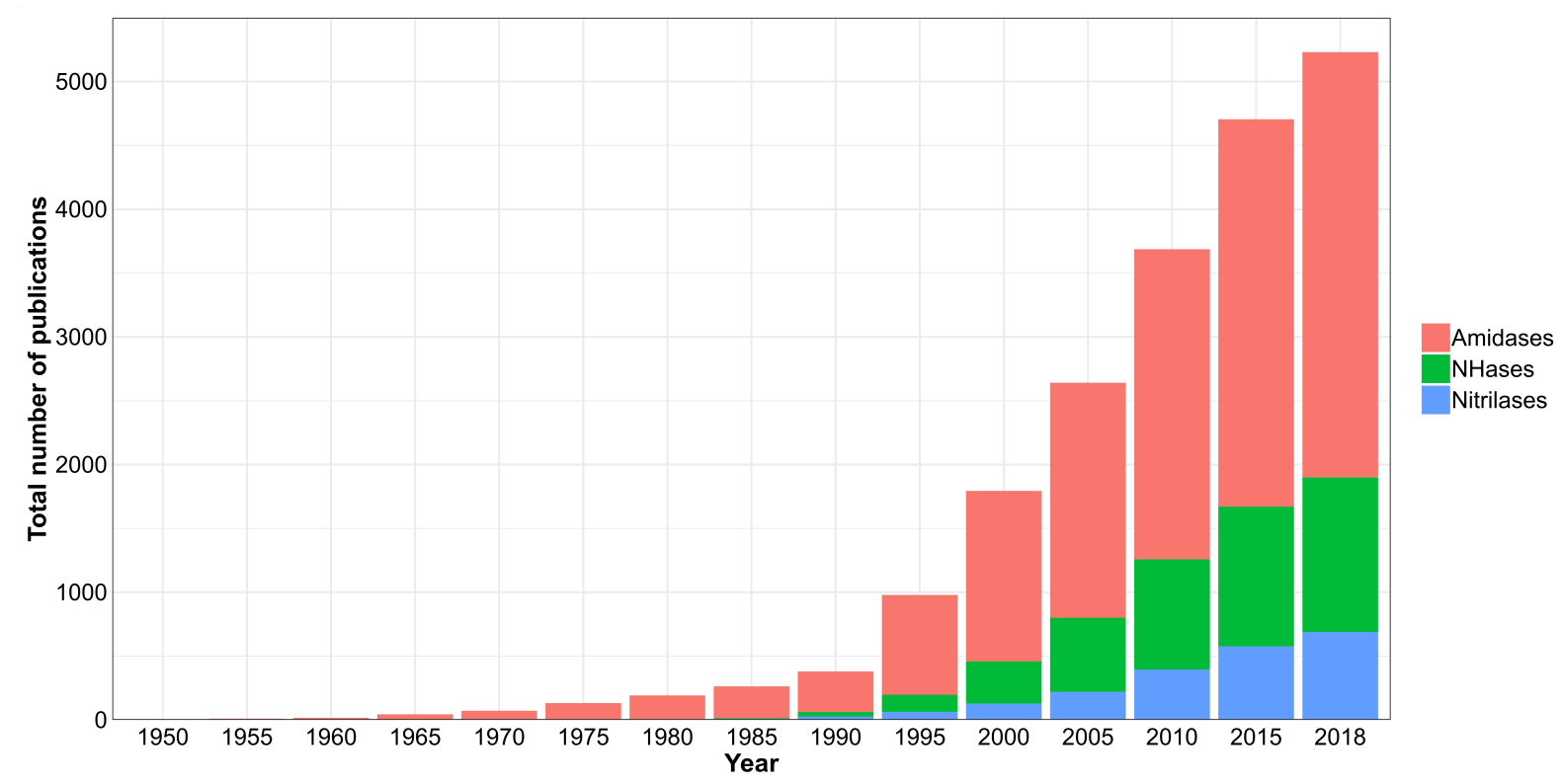

Figure 3: Total number of publications targeting nitrile-degrading enzymes. Data were obtained by search for "amidase", "nitrile hydratase", and "nitrilase" in the Web of Science. Date of search: $12^{\text {th }}$ November 2018.

Nitriles and nitrile-derived products can be found in different fields of industry. In pharmacy, more than 30 drugs containing nitrile groups are used nowadays and several more are in clinical studies. Their spectrum reaches from antidiabetics (vildagliptin) and anticancer drugs (anastrazole) over cardiotonic agents (olprinone) to antidepressants (escitalopram) (103). Probably the most important application in pharmacy is the utilization of a penicillin amidase to produce 6-aminopenicillic acid from penicillin G, allowing the cost-efficient production of penicillin derivates $(104,105)$. In addition, a nitrilase catalyzing the hydrolysis of the industrially produced 2-(4'-isobutylphenyl)propionitrile to ibuprofen is known and leads to high yields of the active isomer (106).

Besides synthesis of small amounts of expensive chemicals, nitriles and nitrile-degrading enzymes are also used for production of bulk chemicals. Companies like BASF AG or Mitsubishi Rayon use NHases for production of more than 600,000 tons acrylamide per year $(107,108)$. Another example for industrially used NHases is the production of 11,500 tons nicotinamide, a form of vitamin B3, by Lonza in 2006 (109). Furthermore, hundreds of tons of mandelic acid and nicotinic acid are produced per year 
using nitrilases $(74,110)$. Especially the synthesis of nicotinic acid is a good example how biocatalysts can help to fulfill the principles of green chemistry. The most common manufacturing process is based on liquid-phase oxidation of 2-methyl-5-ethylpyridine at high temperatures and high pressure (111). In addition, it depends on the use of toxic, flammable, and hazardous nitric acid, which is difficult to handle and a threat to the environment (112). With transition to nitrilase-based production processes, these unfavorable conditions are omitted, leading to a safer and greener chemical industry.

Nitriles are not only industrially important substrates or products, but also used in agriculture. Prominent examples are the herbicides bromoxynil and dichlobenil $(113,114)$, the latter also available for private households. Transgenic oxynil-herbicide resistant crops have been developed to deal with toxic effects (115) but accumulation in the environment could cause problems. In addition, other industrially used nitriles released into (waste)water and soil have deleterious effects on the local biota. Consequently, bioremediation involving nitrile-degrading enzymes is of increasing scientific and economic interest. Classic treatment of nitrile-contaminated industrial waste is performed with chemicals like hydrogen peroxide or by alkaline chlorination, carrying the risk of subsequent contamination with toxic chemicals (116). Therefore, several remediation processes based on enzymatic nitrile degradation have been developed during the last decades. Most of them focus on immobilization of nitrile-degrading bacteria with alginate to simplify operation of decontamination systems $(117,118)$. Another approach uses bacterial consortia consisting of biofilm-forming and nitriledegrading organisms for bioremediation of acetonitrile-containing waste water (119). In addition, a recombinant biofilm-forming Bacillus containing a Rhodococcus nitrilase was created (120). Due to its biofilm, this strain exhibits high resistance to nitrile toxicity and is therefore a promising organism for industrial bioremediation.

\subsection{Enzyme screening methods}

Discovery of novel enzymes is important, especially as not every limitation for industrial application can be solved by protein engineering. Probably the most traditional screening methods are culturebased techniques. For that purpose, a microbial inoculum is grown under presence of a chemical of interest used as e.g. sole carbon source. Only microorganisms with desired enzymatic can grow by metabolization of the added compound and are further analyzed. Similarly, agar plates containing indicator dyes can be used, showing color changes or halos around microorganisms with desired activity $(121,122)$. Despite its simplicity, this technique is highly limited as only $1 \%$ of all microorganisms can be grown under standard laboratory conditions. Therefore, most metabolic potential cannot be harvested by this approach (123). 
As consequence, two screening approaches have been developed to explore the genetic diversity of non-culturable microorganisms. The first method uses gene libraries from metagenomic DNA and activity-based screens. By combining expression of metagenomic genes in hosts like Escherichia coli with simple activity assays like the previously described plate method, novel enzymes can be identified (124-127). The drawback of this system is its high effort and problems with the heterologous expression, as often less than $50 \%$ of the investigated genes are expressible in $E$. coli (128).

The second method uses comparative genomics to mine (meta)genomes for new biocatalysts. For that purpose, (public) sequence data are searched for homologues of an enzyme of interest, often leading to the prediction of several new proteins. Subsequently, these candidates are subcloned into $E$. coli or other hosts and tested for activity. Sequence -driven discovery of many novel enzymes such as methyl halide transferases, mandelate oxidases, rare arylmalonate decarboxylases, and nitrilases demonstrates the potential of this approach (129-132). Nevertheless, identified putative proteins do not necessarily exhibit the targeted function. Furthermore, entirely novel enzymes cannot be identified as only homologues to already known proteins are detected (133).

Besides (meta)genome-based screening approaches, transcriptome-based screenings have been developed recently. By comparison of different growth conditions, differential transcriptomics can help to identify novel enzymes or metabolic pathways. This approach was already used for determination of genes involved in heat-shock response in Brevibacillus borstelensis, adaption of $E$. coli to different toxins, or identification of biocatalysts for synthesis of chiral alcohols in Pseudomonas monteilii (134-136). Major benefit of this approach is the simultaneous detection of several interesting genes at once, also allowing the identification of novel enzymes and entire pathways. Nevertheless, differential transcriptomics for identification of biocatalysts have only been applied to single organisms. Therefore, neither complex interactions between members of microbial communities nor organisms difficult to isolate can be analyzed by this approach.

\subsection{Aim of the thesis}

Aim of this thesis is the establishment of a novel approach for identification of biocatalysts by reverse omics techniques. This approach is based on differential analysis of metatranscriptomic datasets without the necessity of designing a suitable screen for the targeted biocatalyst. For this purpose, microbial communities are incubated in the presence and absence of the target compound. Subsequently, mRNA of both approaches is isolated and sequenced. Ideally for quantification and later identification of full-length genes, the RNA datasets are mapped on a metagenome backbone determined from the same sample. By differential analyses of the metatranscriptomic dataset, genes specifically expressed under the presence of the added compound are identified. 
Nitrilases were chosen as genes of interest. Therefore, growth media for the proposed metatranscriptomic screening approach contain either a nitrile or its degradation product in form of the corresponding carboxylic acid and ammonia. Upregulated genes identified during differential analysis of respective metatranscriptomes should belong to nitrilases, nitrile hydratases, amidases, or unknown resistance mechanisms. Before testing the novel screening approach, toxicity of different nitriles and corresponding carboxylic acids must be determined to avoid unintended lethal effects during screening. With the respective values in hand, the novel screening approach will be tested. A known nitrile-degrading organism will be used to establish growth conditions, sample preparation, and bioinformatic analysis. Subsequently, the optimized parameters will be applied to a complex microbial community, leading to the identification of novel biocatalysts. 
Chapter III

Results 


\title{
3.1. Impact of nitriles on bacterial communities
}

\author{
Richard Egelkamp ${ }^{1}$, Till Zimmermann ${ }^{1}$, Dominik Schneider ${ }^{1}$, \\ Robert Hertel ${ }^{1} \&$ Rolf Daniel ${ }^{1}$
}

Submitted to Applied and Environmental Microbiology

${ }^{1}$ Genomic and Applied Microbiology \& Göttingen Genomics Laboratory, Institute of Microbiology and Genetics, University of Göttingen, Göttingen, Germany

Supplementary data can be found on the enclosed DVD

Author contributions

Performed the experiments: RE, TZ

Analyzed the data: RE, DS, RH

Wrote the publication: $\mathbf{R E}, \mathrm{RH}, \mathrm{RD}$

Conceived the study: RH, RD 


\section{ABSTRACT}

Nitriles are organic molecules with a nitrile group $(-\mathrm{C} \equiv \mathrm{N})$ as functional group and often toxic for living organisms. Detoxification can occur via nitrilases that degrade nitriles directly to carboxylic acids and ammonia, or with nitrile hydratases and amidases that convert nitriles to amides and subsequently to carboxylic acids and ammonia. Despite the knowledge of enzymatic degradation pathways, the influence of these compounds on the composition of bacterial communities has not been studied. Initially, the tolerances of four phylogenetically different bacterial strains without known nitrile detoxification systems (Agrobacterium tumefaciens, Bacillus subtilis, Corynebacterium glutamicum, and Escherichia coli) to the toxic effects of nine different nitriles and the corresponding carboxylic acids were determined. Based on these results, the effect of nitriles on diversity and composition of environmentally-derived bacterial communities was monitored over time by $16 \mathrm{~S}$ rRNA gene ampliconbased and metagenome analyses. Three nitriles (acetone cyanohydrin, 2-phenylpropionitrile, and pyruvonitrile) exhibited a lethal, three (phenylacetonitrile, 4-hydroxybenzonitrile, and cyclohexanecarbonitrile) a growth-suppressing and three (succinonitrile, acetonitrile, and crotononitrile) a growth-promoting effect on the studied communities. Furthermore, each tested nitrile exhibited a specific community-shaping effect, e.g. communities showing growth-suppression exhibited high relative abundance of Paenibacillus. In general, analysis of all data indicated a higher resistance of Gram-positive than Gram-negative bacterial community members and test organisms to growth-suppressing nitriles. Finally, more than 70 putative nitrilase-encoding and more than 20 potential nitrile hydratase-encoding genes were identified during analysis of metagenomes derived from nitrile-enrichments, underlining the high yet often unexplored abundance of nitrile-degrading enzymes.

\section{IMPORTANCE}

In this study, we present a systematic analysis of the influence of different nitriles on a complex microbial community. Significant changes of bacterial community diversity and composition depending on the added nitrile occurred. Furthermore, numerous putative nitrile-decomposing enzymes were identified from metagenomes. Thus, the here presented results reveal the potential impact of nitriles and their degradation products on microbial communities and approaches for its determination. 


\section{INTRODUCTION}

Nitriles are structurally diverse organic molecules with a nitrile group $(-\mathrm{C} \equiv \mathrm{N})$ as functional group. Naturally occurring nitriles are found in bacteria, fungi, plants, and animals as well as in marine and terrestrial habitats (1-4). The most widespread nitriles are cyanogenic glycosides, which often are present in plants (5) and other organisms (6). They can serve as storage form of reduced nitrogen (7) or defense compound when degraded to highly toxic hydrogen cyanide (4).

Toxicity of most nitriles does not originate from the nitrile itself, but from cyanide release due to degradation by e.g. cytochrome P450 in eukaryotes (8-10). Subsequently, cyanide inhibits cytochrome c oxidases and therefore the terminal electron acceptor of the respiratory electron transport chain (11). As cytochrome P450 and cytochrome c oxidase are also present in many bacteria $(12,13)$, a similar mode of action as in eukaryotic organisms is indicated.

Detoxification and degradation are facilitated by two known enzymatic routes. The first involves nitrilases (EC 3.5.5.1), which belong to the carbon-nitrogen hydrolase superfamily and harbor a conserved catalytic triad consisting of glutamic acid, lysine, and cysteine $(14,15)$. These enzymes degrade nitriles directly to carboxylic acids and ammonia. In the second route, nitriles are degraded to amides and subsequently to carboxylic acids and ammonia. The hydration of nitriles to amides is catalyzed by nitrile hydratases (NHases; EC 4.2.1.84), which consist of an $\alpha$ - and $\beta$-subunit and are classified based on the used cofactor as cobalt or ferric NHases. The subsequent hydrolysis of amides to carboxylic acids and ammonia is performed by amidases (EC 3.5.1.4) (16).

Nitriles are also important industrial precursors for the production of bulk chemicals like acrylamide (17) or part of various pharmaceuticals such as the anti-cancer drug letrozole or etravirine for HIV treatment (18). Non-intended release of man-made nitriles can lead to environmental accumulation and impact natural communities. In addition, some nitriles are used as herbicides, e.g. dichlobenil (19) or bromoxynil (20). The effect of these synthetic compounds on bacterial communities, the key players in most nutrient cycles (21-23), is almost unknown. While toxicity values and effects of many nitriles on mammals have been reported, comparable values are almost unavailable for microorganisms $(8,11,24,25)$.

The aim of this work was to investigate the effect of nine nitriles and their corresponding degradation products on bacterial communities. Initially, the toxicity of these nitriles and their corresponding carboxylic acids were explored using four bacterial test strains containing no known nitrile detoxification system. Based on these results a community growth experiment was performed and revealed the specific impact of each nitrile on a bacterial community with respect to composition and diversity. Additionally, metagenome sequences provided insight into potential mechanisms of nitrile stress management, as quantity and distribution of nitrile-degrading enzymes were analyzed. 


\section{RESULTS}

Limits for bacterial life. To establish stable nitrile-containing mixed cultures it is necessary to determine the toxic effect of these compounds. Therefore, we analyzed the influence of a set of nine nitriles on the four model organisms Agrobacterium tumefaciens, Bacillus subtilis, Corynebacterium glutamicum, and Escherichia coli by using agar plates containing a rich complex or a minimal medium with different nitrile concentrations. All four tested strains do not contain any known nitriledegradation pathway. As carboxylic acids, the products of a potential nitrile detoxification reaction, also affect microbial growth, their influence was tested in addition.

Growth was monitored for four days on solid agar plates supplemented with a nitrile or the corresponding carboxylic acid and examined every 24 hours (Data Set S1). In general, the Grampositive strains (B. subtilis and C. glutamicum) showed better growth at higher nitrile and carboxylic acid concentrations than the Gram-negative strains (A. tumefaciens and E. coli). Some tested strains revealed different behavior with respect to the nitriles and the corresponding carboxylic acids. For example, 4-hydroxybenzonitrile suppressed the growth of $A$. tumefaciens and $E$. coli even at $5 \mathrm{mM}$ concentration but all organisms except $A$. tumefaciens were able to grow at $25 \mathrm{mM}$ of the corresponding carboxylic acid (Table 1). The higher susceptibility of the tested Gram-negative strains was also observed for pyruvonitrile, as growth was inhibited at 15, 20, and $25 \mathrm{mM}$ concentration, whereas Gram-positive strains grew at $15 \mathrm{mM}$. In addition, cyclohexanecarbonitrile had no influence on the growth of all tested organisms, but cyclohexanecarboxylic acid inhibited growth at $10 \mathrm{mM}$.

The results provided a first insight into the inhibitory potential of the nitriles and carboxylic acids with respect to bacterial growth. The highest non-inhibitory concentration considering all four test strains was chosen for further experiments. In cases where the highest concentration of a nitrile and the corresponding carboxylic acid differed, the lower concentration was chosen for both substances. For substances inhibiting growth even at lowest tested concentration, $5 \mathrm{mM}$ were used subsequently. 


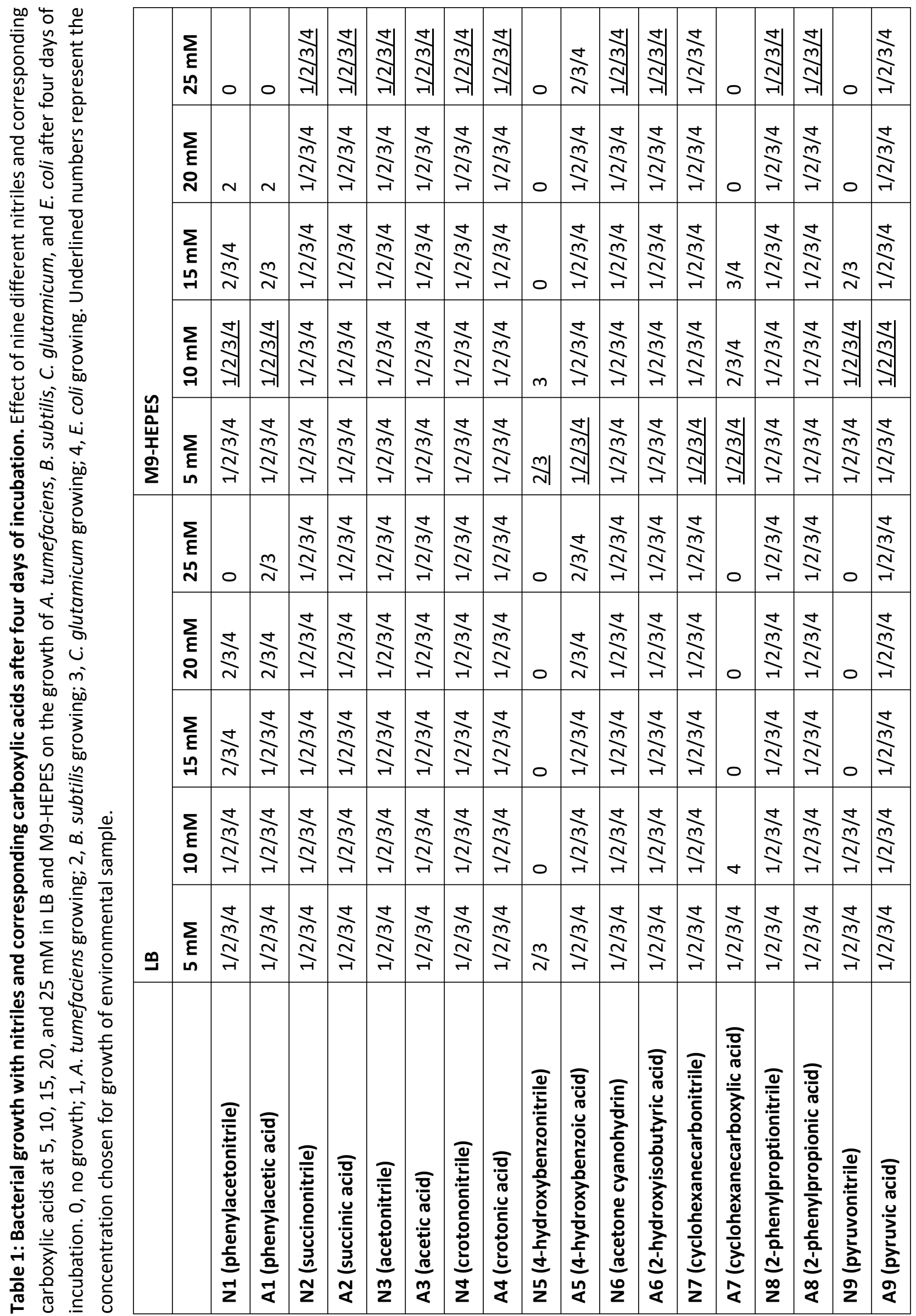


Nitrile and carboxylic acid impact on bacterial growth. To determine bacterial community alterations under the influence of nitriles and their corresponding carboxylic acids a growth experiment in liquid minimum medium was performed. A compost sample from the Experimental Botanical Garden Göttingen (Germany) served as inoculum.

A general reduction of the final OD with each cultivation passage was observed for the nitrile-based and the carboxylic acid-based cultures (Figure 1). At the last measuring point of the carboxylic acidsupplemented cultures, seven of nine conditions revealed higher optical densities than the control, thereby indicating beneficial effects of the supplemented carboxylic acids. In case of the nitrilesupplemented cultures only three nitriles (succinonitrile, acetonitrile, and crotononitrile) exhibited a growth-supporting effect in comparison to the control without nitriles. The beneficial impact of acetonitrile was already obvious from the second passage on. Addition of cyclohexanecarbonitrile did not affect growth, whereas a negative effect on microbial growth was observed for phenylacetonitrile and 4-hydroxybenzonitrile. In contrast, cultures supplemented with acetone cyanohydrin, 2-phenylpropionitrile, or pyruvonitrile showed no growth at all after the first passage.
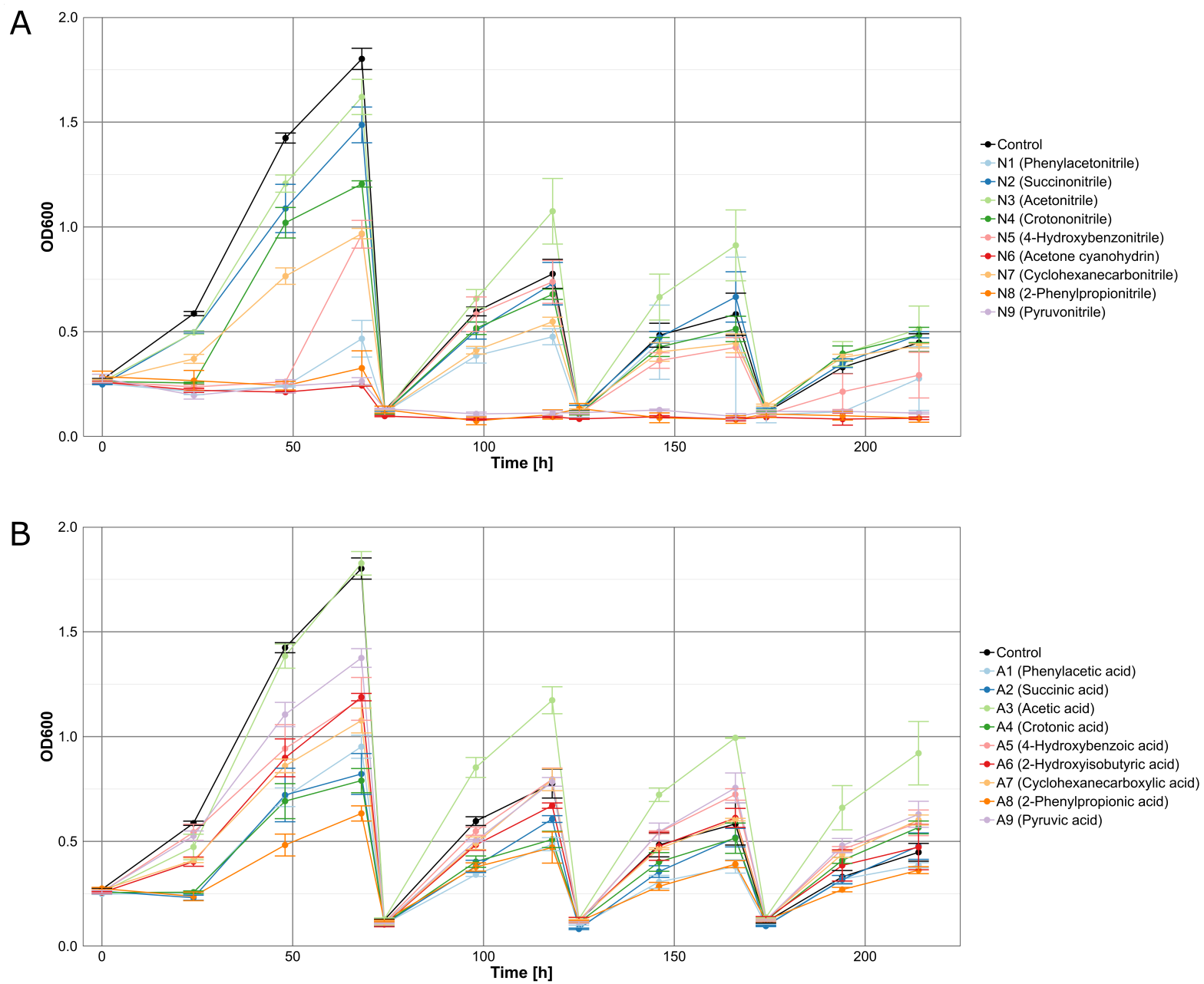

Figure 1: Growth of a microbial community in M9-HEPES treated with (A) nitriles or (B) the corresponding carboxylic acids. 
Bacterial community composition and diversity. The bacterial community composition of the control cultures without addition of nitriles or the corresponding carboxylic acids was determined via amplicon-based 16S rRNA gene analysis (Fig. 2 and Data Set S2). Analysis of Simpson indices of diversity (SID) at genus level revealed reduction of diversity from inoculum (SID 0.98) to the first sampling of the control after $68 \mathrm{~h}$ of incubation (SID 0.58 ). Diversity increased again after $118 \mathrm{~h}$ of incubation (SID 0.78) and stabilized in the later taken control samples (166 and 214 h; SID 0.91 in both cases). Besides inoculum and control cultures, only communities showing growth after $214 \mathrm{~h}$ with the nitrile or the corresponding carboxylic acid were analyzed by $16 \mathrm{~S}$ rRNA gene analysis (Figure 2). Cultures supplemented with acetone cyanohydrin, 2-phenylpropionitrile, and pyruvonitrile were excluded from this analysis due to absence of viability after first passage. Consequently, the respective carboxylic acid-supplemented cultures were also removed, as in these cases comparison between a nitrile and its carboxylic acid was not feasible.

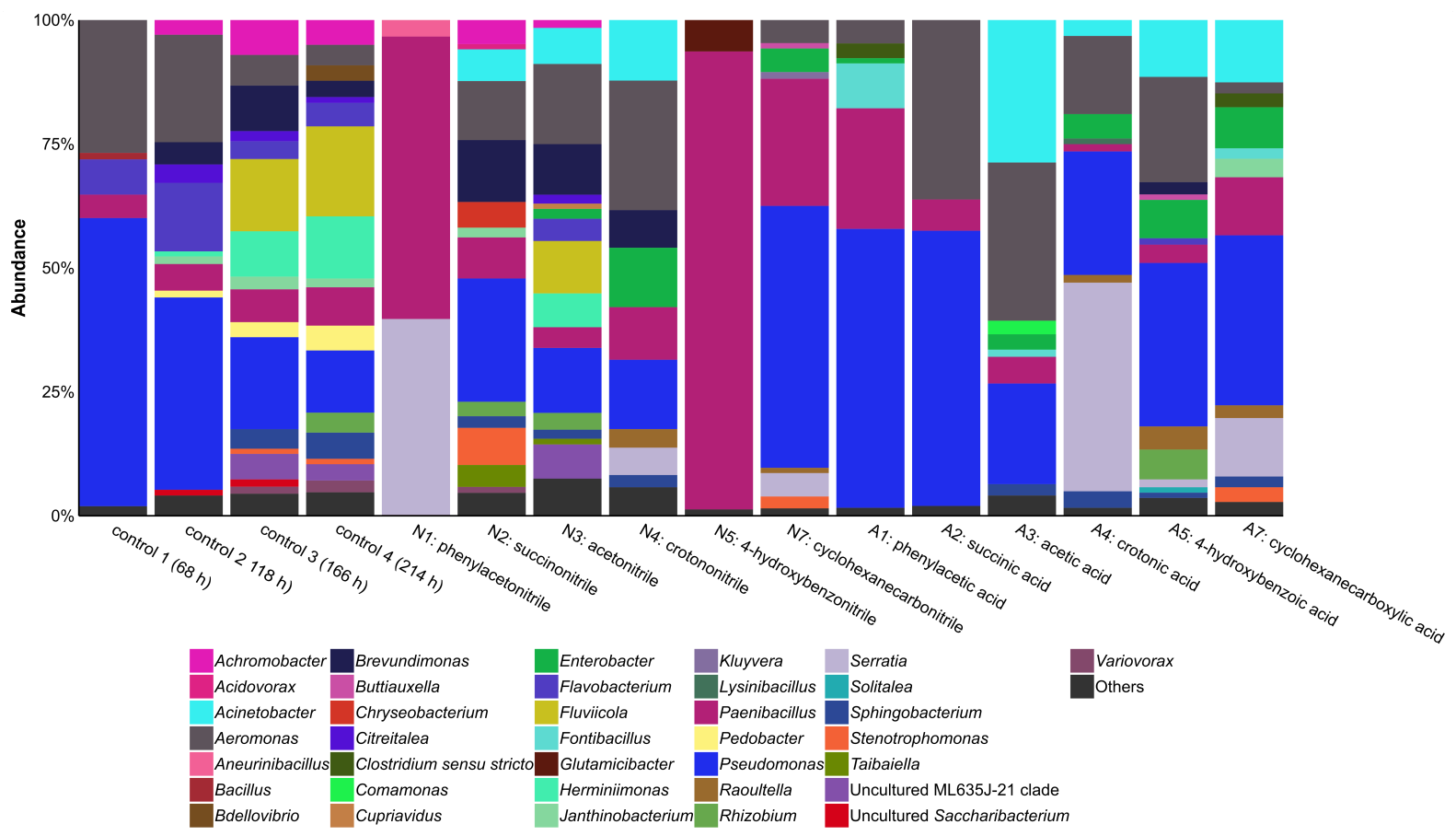

Figure 2: Abundance of bacterial genera based on 16S rRNA gene analysis in cultures containing different nitriles or carboxylic acids after $214 \mathrm{~h}$ of incubation. Others, genera with a relative abundance of $>1 \%$.

Nitrile-supplemented cultures indicated a general formation of two distinct bacterial community types. Cultures containing succinonitrile, acetonitrile, or crotononitrile showed a high diversity (SID 0.88, 0.91 and 0.86 , respectively) when compared to cultures with phenylacetonitrile, 4-hydroxybenzonitrile or cyclohexanecarbonitrile (SID 0.52, 0.14 and 0.65, respectively). Cultures supplemented with the corresponding carboxylic acids exhibited a similar pattern. Communities with acetic acid (SID 0.77), crotonic acid (SID 0.73), 4-hydroxybenzoic acid (SID 0.82), and 
cyclohexanecarboxylic acid (SID 0.83) exhibited a higher diversity compared to cultures supplemented with phenylacetic acid (SID 0.61) and succinic acid (SID 0.56). In addition, some carboxylic acidcontaining cultures showed strong variations in diversity in relation to the corresponding nitrilecontaining cultures. In case of succinonitrile and succinic acid, diversity decreased from SID 0.88 to 0.56. A similar trend was observed for acetonitrile (SID 0.91) and acetic acid (SID 0.77). In contrast, a highly increased diversity could be detected in 4-hydroxybenzoic acid-containing cultures (SID 0.82) compared to the corresponding nitrile (SID 0.14). These differences in diversity indicate a specific effect of the nitrile group in comparison to the carboxylic acid group.

Nitrile-affected cultures showed distinct communities when compared with the carboxylic acid counterparts. Pseudomonas was present under most conditions, except in cultures containing phenylacetonitrile or 4-hydroxybenzonitrile. A similar behavior was recorded for Aeromonas. Interestingly, Paenibacillus is the dominant genus in phenylacetonitrile- or 4-hydroxybenzonitrilecontaining cultures with relative abundances of $57 \%$ and $92 \%$, respectively. Members of this genus are also present in all other cultures but in lower relative abundances. In addition, Brevundimonas was present ( $>1 \%$ relative abundance) in three of the six nitrile-treated cultures, but only in one containing a carboxylic acid. Furthermore, Acinetobacter and Aeromonas showed an on average higher relative abundance under acetic acid treatment. In contrast, Pedobacter was only identified in the controls ( $>1 \%$ relative abundance), while Aneurinibacillus, Chryseobacterium, and Glutamicibacter were only present after treatment with phenylacetonitrile, succinonitrile or 4-hydroxybenzonitrile, respectively. Finally, Serratia showed high relative abundance in cultures containing phenylacetonitrile $(40 \%)$ or crotonic acid (42\%).

The combination the 16S rRNA gene analysis with the corresponding growth curves revealed further linkages between the density of a culture and its diversity. Cultures with phenylacetonitrile and 4-hydroxybenzonitrile did not only reveal reduced optical density, but a reduced diversity when compared to the cultures containing the corresponding carboxylic acids. In contrast, succinic acidcontaining cultures showed low diversity, but exhibited higher density than the control. Finally, several cultures (succinonitrile, acetonitrile, 4-hydroxybenzoic acid, and cyclohexanecarboxylic acid) showed higher density and higher diversity than the control, indicating a growth-benefiting effect of the added chemicals. 
How to handle nitriles. To assess in which way the different bacterial communities manage the supplemented nitriles, metagenomes of all cultures incubated for $214 \mathrm{~h}$ and showing growth were sequenced. Subsequently, the metagenomes were assembled, annotated, and analyzed. The samples were checked for presence of genes encoding nitrilases and nitrile hydratase subunits. Finally, the taxonomic assignment of the contigs harboring the targeted genes was used to infer the phylogenetic origin of the deduced enzymes (Data Set S3).

In general, the metagenomes showed a similar taxonomic composition as previously determined via $16 \mathrm{~S}$ rRNA gene analysis. Other domains were barely present, as only three respective microorganisms were detectable (Figure 3A). The first is the nematode Parastrongyloides, whose DNA was detected in the control culture after $166 \mathrm{~h}$ of incubation (2\%) as well as in cultures with succinonitrile (4\%), acetonitrile (4\%), and crotononitrile ( $8 \%)$. The second is a Spounalikevirus with a relative abundance of $8 \%$ in the culture containing 4-hydroxybenzonitrile. Third, a green alga belonging to the genus Pyramimonas was detected in cyclohexanecarbonitrile-containing cultures with $2 \%$ relative abundance.

Analysis of nitrile-degrading enzymes encoded by the metagenomes revealed a broad distribution. Especially members of the genus Pseudomonas harbor several potential genes encoding nitrilases and NHases in nitrile- and carboxylic acid-supplemented cultures (Figure 3A). Furthermore, the untreated control cultures showed high abundance of nitrile-degrading enzymes when compared to other treatments (Figure 3B).

Regarding a potential impact of a nitrile on a bacterial community, several effects were observed. For example, the abundance of Pseudomonas increased in the cyclohexanecarbonitrile-containing culture compared to its carboxylic acid counterpart, while the diversity of putative nitrilases was reduced by $50 \%$ (Fig. 3A). At the same time, abundance of nitrilase genes increased almost fourfold, indicating nitrile-induced enrichment of nitrile-converting genes and correspondingly enrichment of nitriledegrading capacity of the community (Figure 3B). 

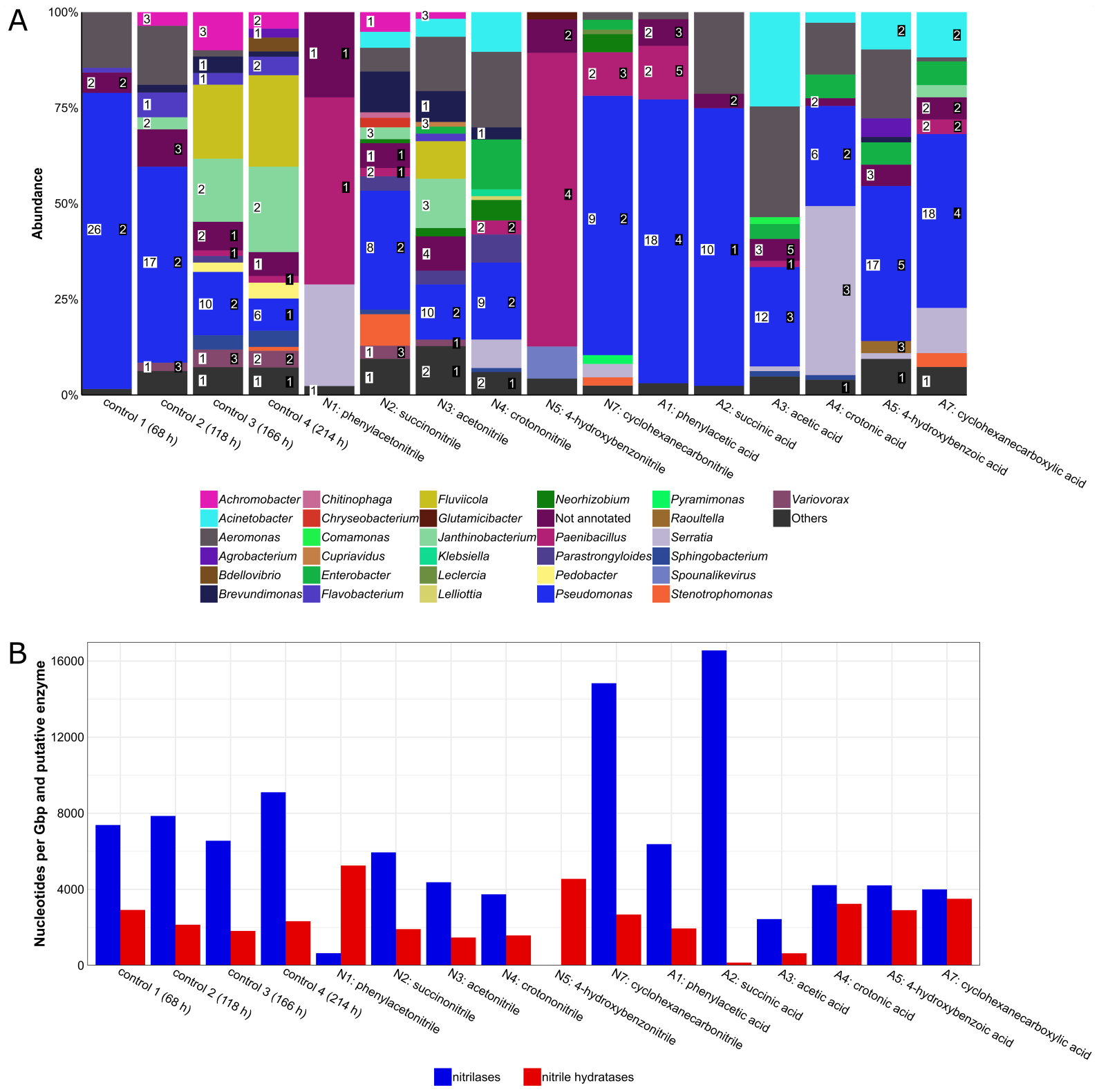

Figure 3: Putative nitrilases and nitrile hydratases found in metagenomes of enrichment cultures. A, Distribution of enzymes. Numbers in white box represent number of nitrilases and numbers in black box represent number of nitrile hydratase subunits. Others, genera with an abundance of $>1 \%$. B, Gene abundance based on coverage. 


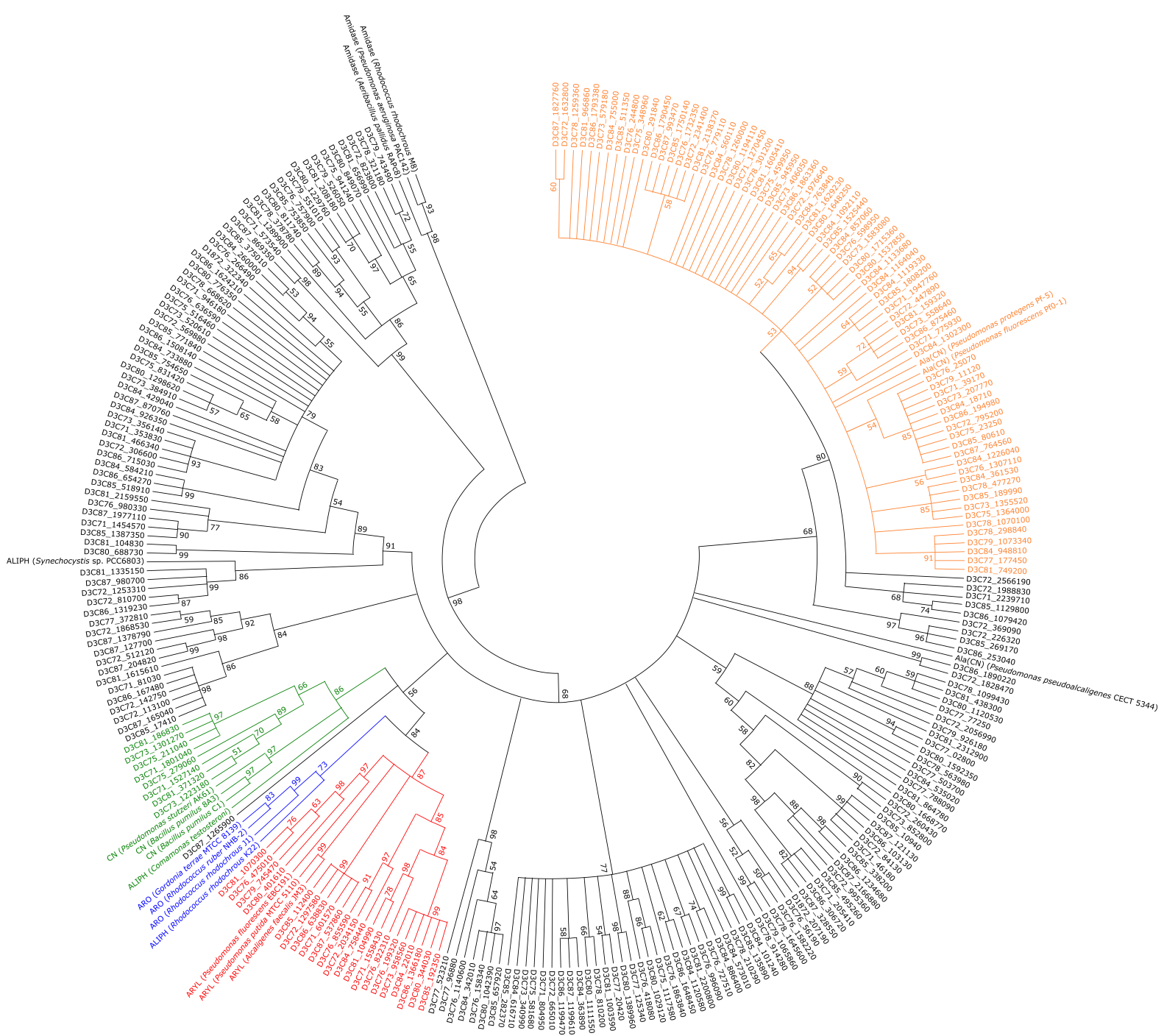

Figure 4: Phylogenetic tree containing all putative metagenome-derived nitrilases. 500 bootstrap replicates were calculated and tree nodes were condensed at values below $50 \%$. ALIPH, aliphatic nitrilase; ARO, aromatic nitrilase; ARY, arylacetonitrilase; ALA(CN), $\beta$-cyano-L-alanine-nitrilase; CN, cyanide dihydratase. Green, putative cluster of cyanide dihydratases; blue, putative cluster of aromatic nitrilases; red, putative cluster of arylacetonitrilases; orange, putative cluster of $\beta$-cyano-L-alaninenitrilases.

A phylogenetic tree containing all identified and reference nitrilases (Figure 4) revealed no treatmentspecific clusters. Approximately $25 \%$ of all putative enzymes group together with $\beta$-cyano-L-alaninedegrading nitrilases. In addition, small clusters of arylacetonitrilases and cyanide dihydratases were found, containing 21 or 8 of the putative nitrilases, respectively. For the remaining 156 putative nitrilases, specific clustering was not recorded. 


\section{DISCUSSION}

Studies on the susceptibility of prokaryotes to several bioactive compounds such as antibiotics (26), cytostatics (27), fungicides (28), insecticides (29), and nematicides (30) are performed frequently. To our knowledge literature addressing bacterial sensitivity towards nitriles and their degradation products has not been published. Toxicity data, determined for A.tumefaciens, B. subtilis, C. glutamicum and E. coli, indicate several differences in their susceptibility. Both Gram-positive strains revealed increased tolerance to possible growth-inhibiting effects of nitriles compared to the Gramnegative strains, whereby $A$. tumefaciens was most susceptible.

Among the tested compounds, 4-hydroxybenzonitrile was the most toxic. As the closely related compounds 3,5-diiodo-4-hydroxybenzonitrile (ioxynil) and 3,5-dibromo-4-hydroxybenzonitrile (bromoxynil) are common herbicides $(31,32)$, a certain toxicity to microorganisms could be inferred. Especially bromoxynil has a documented negative impact on the soil microbial biomass (33). The second most toxic chemical was pyruvonitrile, which has structural similarities to the key metabolite pyruvate. This similarity leads to interference with central metabolism and deleterious effects. The toxicity of the carboxylic acids was generally reduced compared to the corresponding nitriles, except for cyclohexanecarboxylic acid. Its toxicity was significantly higher for the tested prokaryotes than that of its nitrile counterpart. The respective mechanism remains unknown but underlines the importance to investigate also potential degradation products of a nitrile.

The toxicity assay served mainly as an initial test to define working concentrations for the investigation of bacterial communities. Optical density data, representative for the viability of a community, underlined the value of the liquid culture setup. In case of the tested nitriles, only succinonitrile, acetonitrile and crotononitrile (Fig. 1A) exhibited a positive effect on microbial growth. This indicates that the predominat bacterial community members of these cultures possess the ability to degrade and utilize these nitriles. For acetonitrile this effect was already experimentally confirmed through the successful isolation of nitrile-degrading strains from similar cultures (34). All other nitrile-containing cultures revealed reduced density indicating reduced degradation ability or tolerance levels with respect to the tested compounds.

For the differences between the solid and liquid cultures with the substrates acetone cyanohydrin, 2-phenylpropionitrile, and pyruvonitrile, two explanations could be assumed. (1) In case of cyanohydrins, spontaneous decomposition has been described under different conditions $(35,36)$, and probably similar mechanisms also apply for other nitriles. Consequently, different stabilities of the nitriles under both tested conditions could have had influence on their effect. (2) The compost sample did not contain any acetone cyanohydrin, 2-phenylpropionitrile or pyruvonitrile resistant or degrading microorganisms. However, 16S rRNA gene analysis of the inoculum revealed the presence of 
A. tumefaciens, B. subtilis, C. glutamicum, and E. coli, species successfully grown during the initial plate test and thus making the first assumption more likely. Furthermore, degrading organisms or at least nitrilases for acetone cyanohydrin and 2-phenylpropionitrile have already been identified (37-44), indicating the existing potential to handle these chemicals.

Monitoring diversity of the complex communities via 16S rRNA gene analysis revealed a reduction of diversity in the liquid cultures compared to the inoculum. As often more than $99 \%$ of all environmental microorganisms cannot be cultivated under laboratory conditions (45-47), a decline was expected. Generally, the 16S rRNA gene analysis of the control samples confirmed the experimental setup as appropriate and sufficient to establish a stable community. Re-diversification of the controls over the course of the experiment is explained by the prolonged incubation time. Extended incubation is a wellknown factor for increasing the number of isolates on solid media (46). Apparently, regular transfer to fresh medium and therefore prolonged incubation without nutrient deficiency leads also to an increase of proliferating and fast-growing bacteria in liquid medium. Slow-growing organisms get the possibility to overcome their lag period and proliferate during longer incubation times. This re-diversification has a severe impact on results using liquid cultures, as several genera would be missed in case of earlier sampling and analysis. In this experiment, diversity stabilized between the third and fourth sampling time. The $16 \mathrm{~S}$ rRNA gene data after $214 \mathrm{~h}$ of incubation revealed diverse individual cultures, stating that nitriles and their degradation products significantly impact bacterial community composition and diversity. In accordance with initial toxicity test, we could confirm an increased relative abundance of Gram-positive genera in cultures containing growth-suppressing nitriles such as phenylacetonitrile, 4-hydroxybenzonitrile and cyclohexanecarbonitrile. It appears that these organisms are more resistant to the negative influence of these specific compounds. Nevertheless, high abundance of Paenibacillus in the 4-hydroxybenzonitrile-containing sample was unexpected, as we could not find any confirmed nitrile-degrading enzyme for this genus in literature. However, putative nitrilase-encoding genes are present in publicly available Paenibacillus genomes. In addition, closely related cyanide dihydratases were just recently predicted for this genus (48). For Brevundimonas, also showing higher abundances in the nitrile-containing communities compared to the carboxylic acid-containing ones, database entries or literature on nitrile-degrading enzymes were not found. Nitrile degradation has been described for Serratia $(49,50)$, but potential nitrile-converting genes phylogenetically assigned to this genus were not predicted in the metagenome derived from the phenylacetonitrile-treated culture. Nevertheless, with an abundance of $40 \%$ in the respective culture, members of the Serratia genus seem to be well-adapted to phenylacetonitrile. Therefore, probably other mechanism lead to the nitrile tolerance. Thus, further investigation of all three genera could be of great value for the discovery of novel nitrile-degrading enzymes and resistance mechanisms. 
A few reports mentioned nitrile-degrading activity of Acinetobacter $(51,52)$, but not for Aeromonas. The absence of putative nitrilases and nitrile hydratases for Aeromonas and only few nitrile hydratase subunits assigned to Acinetobacter indicate the rarity of respective genes in both genera. The reduced average abundance of both genera in nitrile-treated cultures is probably explained by a higher susceptibility to possible toxic effects of the nitriles and subsequent displacement of these genera by better adapted microorganisms such as Paenibacillus and Brevundimonas.

Pedobacter is known for broad antibiotic resistance potential and heavy metal tolerance (53-55), but it seems like this genus is not adapted to nitrile-harboring or carboxylic acid-containing environments. In addition, genes encoding putative nitrilases or nitrile hydratases were not predicted to be derived from members of this genus. Interestingly, the same applies for putative nitrile-degrading enzymes derived from Aneurinibacillus, Chryseobacterium, and Glutamicibacter, but every genus shows growth in one nitrile-containing culture. Thus, the presence of so far unknown nitrile resistance or degradation mechanisms is indicated.

It is doubtful that with Parastrongyloides a parasite of Australian possums was living in German compost, but probably other related nematodes present in this environment could be able to survive in M9-HEPES, especially as Parastrongyloides shows growth in liquid medium (56).

The assumption that nitriles and their handling are a common feature in nature (1-7) could be confirmed as many nitrile-degrading enzymes are commonly present in different genera. They could even be frequently identified in the control culture facing neither the nitrile itself nor the potential degradation product. Of special interest for further investigations could be the high abundance of nitrilase-encoding genes in the cyclohexanecarbonitrile-containing culture, demonstrating enrichment of respective genes. This could be an indicator for proliferation of a nitrile-degrading bacterium and consequently for active nitrile degradation.

Phylogenetic analysis revealed a high diversity of the encountered putative nitrilases as they were assigned to several different groups. The close clustering of aromatic nitrilases and arylacetonitrilases was expected as both groups act on substrates containing aromatic structures. The $R$. rhodochrous $\mathrm{K} 22$ nitrilase preferably acts on aliphatic nitriles but is similar to benzonitrile-degrading nitrilases with respect to sequence (57). This could explain its clustering with other aromatic nitrilases, even though it has a different substrate specificity. 
Conclusion and outlook. The here employed experimental setup for the investigation of the impact of nitriles and their degradation products on bacterial communities showed that these compounds significantly influence the composition of bacterial communities and partially lead to a strong reduction of diversity. In addition, it revealed strains with potential for remediation purposes such as Paenibacillus in case of 4-hydroxybenzonitrile.

In conclusion, this work provides a first insight on the influence of different nitriles and their rcorresponding carboxylic acids on microorganisms. A different susceptibility of Gram-negative (A. tumefaciens and E. coli) and Gram-positive (B. subtilis and C. glutamicum) test strains was observed during toxicity tests, but the number of tested organisms and chemicals should be increased to provide insights in the underlying mechanisms. The liquid culture experiment with a compost community as inoculum highlighted problems when transferring complex communities for several times to fresh medium, as the maximal optical densities were reduced each passage. Nevertheless, the analysis of the control cultures over time revealed a diversification and stabilization of the bacterial community composition, indicating that the time point for analysis of the chemically-treated cultures was appropriate. In addition, the toxic effects of certain nitriles (acetone cyanohydrin, 2-phenylpropionitrile, and pyruvonitrile) for microbial communities could be documented. Prevention of environmental contamination with these compounds seems therefore important, as bioremediation might be problematic. At the same time, lethal effects of the corresponding carboxylic acids were not observed, indicating specific toxicity of the nitriles.

The comparison of the cultures under the influence of nitriles with the cultures containing the respective carboxylic acids revealed many differences. The nitrile group exhibited a different effect on microorganisms than the acid counterpart. As some carboxylic acids show higher growth suppression and a strong influence on community composition, it seems reasonable to study the influence of educts and products of an enzymatic reaction. This should preferably be done before starting with enrichment or screening experiments for organisms harboring the corresponding enzymes to avoid unintended lethal effects. Especially putative nitrilases clustering with already characterized enzymes in the here presented phylogenetic tree are interesting candidates for further investigation, as chances for enzymatic activity are high. Additionally, a broader picture of nitrile influence on the nitrile-treated cultures could be gained by metatranscriptomic studies, as these have the potential to reveal stress responses, resistance mechanisms and novel degradation strategies. This knowledge could help developing novel or better biotransformation and bioremediation strategies and finally lead to a reduction of environmental contamination. 


\section{MATERIAL AND METHODS}

Media and stock solutions. For growth of microorganisms, LB-Miller medium (10 g tryptone, $10 \mathrm{~g} \mathrm{NaCl}$ and $5 \mathrm{~g}$ yeast extract per liter) (58) or M9-HEPES was used. M9-HEPES was prepared using $15.5 \mathrm{~g}$ HEPES, $1 \mathrm{~g} \mathrm{NH}_{4} \mathrm{Cl}$ and $0.5 \mathrm{~g} \mathrm{NaCl}$ per liter. The $\mathrm{pH}$ was adjusted to 7.0 before autoclaving. Ten $\mathrm{ml}$ ATCC vitamin supplement (LGC Standards, Teddington, UK), $10 \mathrm{ml}$ ATCC trace mineral supplement, $10 \mathrm{ml}$ glucose solution (20\%), $1 \mathrm{ml} \mathrm{MgSO}_{4} \times 7 \mathrm{H}_{2} \mathrm{O}$ solution (1 M), $1 \mathrm{ml} \mathrm{CaCl}$ solution $(14.7 \mathrm{~g} / \mathrm{l})$ and $1 \mathrm{ml}$ thiamine-HCl solution $(1 \mathrm{mg} / \mathrm{ml})$ were added afterwards. For toxicity determination, following chemicals were solved in $\mathrm{H}_{2} \mathrm{O}$, DMSO, or ethanol and added to the medium: phenylacetonitrile (N1), succinonitrile (N2), acetonitrile (N3), crotononitrile (N4), 4-hydroxybenzonitrile (N5), acetone cyanohydrin (N6), cyclohexanecarbonitrile (N7), 2-phenylpropionitrile (N8), pyruvonitrile (N9), phenylacetic acid (A1), succinic acid (A2), acetic acid (A3), crotonic acid (A4), 4-hydroxybenzoic acid (A5), 2-hydroxyisobutyric acid (A6), cyclohexanecarboxylic acid (A7), 2-phenylpropionic acid (A8), and pyruvic acid (A9) (all chemicals from Sigma-Aldrich, Taufkirchen, Germany and TCI Chemicals, Eschborn, Germany). In case of carboxylic acids, an equimolar amount of ammonia was added in form of ammonia water (Carl Roth, Karlsruhe, Germany). For agar plates, $15 \mathrm{~g}$ agar per liter was added.

Nitrile toxicity tests on selected strains. Agrobacterium tumefaciens NTL4(pCF372)(pCF218) (59), Bacillus subtilis NCIB 3610 (60), Corynebacterium glutamicum ATCC 13032 (61) and Escherichia coli W3110 were used as model organisms during this study. Precultures of these strains were grown in LB medium at $30^{\circ} \mathrm{C}$ and $180 \mathrm{rpm}$ (Multitron shaker, Infors, Einsbach, Germany) for $24 \mathrm{~h}$. Afterwards, each strain was plated on a quadrant of a LB or M9-HEPES plate containing one of the nine nitriles or the respective carboxylic acid in a concentration of 5, 10, 15, 20 or $25 \mathrm{mM}$. As control, plates with LB or M9-HEPES were used. Every $24 \mathrm{~h}$, the growth on the plates was analyzed and categorized on a scale of 0 to 4 by comparison with the control. In case of no growth a 0 was given, whereas similar growth compared to the control was treated as 4 . The remaining values were given for approx. $25 \%(=1), 50 \%$ $(=2)$ and $75 \%(=3)$ of colony size and opaqueness when compared to the control.

Nitrile toxicity on an environmental community. Compost (100 g, pH 7.5) of the Experimental

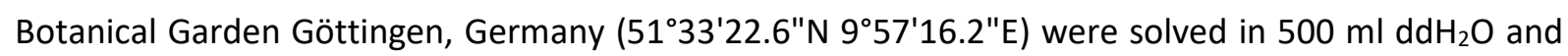
subsequently filtered with a $2.7 \mu \mathrm{m}$ GF/D glass fiber filters (Whatman, Little Chalfont, UK). Baffled flasks (total volume $100 \mathrm{ml}$ ) were filled with $35 \mathrm{ml} \mathrm{M9-HEPES} \mathrm{containing} \mathrm{one} \mathrm{of} \mathrm{the} \mathrm{nitriles} \mathrm{or} \mathrm{carboxylic}$ acids in the above-mentioned tested concentrations (Table 1). Flasks filled only with M9-HEPES served as control. All conditions were prepared in triplicates. The solved and filtered compost $(5 \mathrm{ml})$ were added to each baffled flask and the cultures were incubated at $25^{\circ} \mathrm{C}$ and $75 \mathrm{rpm}$ (Multitron shaker). To monitor the growth of the cultures, the optical density at $600 \mathrm{~nm}\left(\mathrm{OD}_{600}\right)$ was measured. Two prevent starvation of the microorganisms, cultures were transferred after three days to fresh medium 
containing the same nitrile or carboxylic acid at the concentrations used before. The new cultures were adjusted to an $\mathrm{OD}_{600}$ of 0.1 and incubated again at $25^{\circ} \mathrm{C}$ and $75 \mathrm{rpm}$. In case that cultures showed no growth, the cultures were not transferred to fresh medium but further incubated. The procedure was repeated two additional times after two days of incubation per growth period. The final cultures were again incubated for two days.

DNA extraction. DNA of the compost sample solved in water was isolated before and after filtering using the PowerMax soil DNA isolation kit (MO BIO Laboratories, Carlsbad, CA, USA) according to the manufacturer's protocol. DNA of the M9-HEPES-containing enrichment cultures was isolated using the MasterPure Complete DNA and RNA purification kit as recommended by the manufacturer (Epicentre, Madison, WI, USA). Concentration of isolated DNA was measured using a NanoDrop ND-1000 (Thermo Fisher Scientific, Waltham, MA, USA) or with a Quant-iT dsDNA HS assay kit and a Qubit fluorometer as recommended by the manufacturer (Invitrogen, Carlsbad, CA, USA).

$16 \mathrm{~S}$ rRNA gene sequencing. For amplification of the V3-V4 region of bacterial 16S rRNA genes, the primers S-D-Bact-0341-b-S-17 and S-D-Bact-0785-a-A-21 (62) were used. The primers contained adapters for Illumina MiSeq sequencing (Ilumina, San Diego, CA, USA). The DNA isolated from solved compost or M9-HEPES-containing enrichment cultures was used as template. PCR reaction mixtures (50 $\mu$ l final volume) contained $10 \mu \mathrm{l} 5$-fold Phusion GC buffer, $0.2 \mu \mathrm{M}$ of each of the primers, $0.2 \mu \mathrm{M}$ $\mathrm{MgCl}_{2}, 2.5 \mu \mathrm{l}$ DMSO, $200 \mu \mathrm{M}$ of each of the four dNTPs, $1 \mathrm{U}$ of Phusion DNA polymerase (Thermo Fisher Scientific), and $25 \mathrm{ng}$ template DNA. For amplification, the following protocol was used: initial denaturation at $98^{\circ} \mathrm{C}$ for $1 \mathrm{~min}, 25$ cycles of denaturation at $98^{\circ} \mathrm{C}$ for $45 \mathrm{~s}$, annealing at $60{ }^{\circ} \mathrm{C}$ for $45 \mathrm{~s}$, and elongation at $72{ }^{\circ} \mathrm{C}$ for $30 \mathrm{~s}$, followed by final elongation at $72{ }^{\circ} \mathrm{C}$ for $5 \mathrm{~min}$. PCR reactions for each culture were performed in triplicate, pooled in equal amounts, and purified with the NucleoMag 96 PCR kit (Macherey-Nagel, Düren, Germany) according to the manufacturer's protocol. For quantification of the PCR products, a Qubit fluorometer was used in combination with the Quant-iT dsDNA HS assay kit as recommended by the manufacturer (Invitrogen). The purified amplicons were indexed using the Nextera XT DNA library prep kit according to the instructions of the manufacturer (Illumina). Dual index paired-end sequencing ( $2 \times 300$ bp) with v3 chemistry was done on an Illumina MiSeq platform as recommended by the manufacturer (Illumina).

165 rRNA gene sequence analysis. CASAVA data analysis software (Illumina) was used for demultiplexing and clipping of sequence adapters from raw sequences. Before removing sequences with an average quality score below 20 and unresolved bases with split_libraries_fastq.py from QIIME 1.9.1 (63), paired-end sequences were merged using PEAR v0.9.10 with default parameters (64). Default settings of cutadapt 1.14 (65) were used for removal of non-clipped reverse and forward primer sequences. Subsequently, the UNOISE2 pipeline of USEARCH 9.2.64 (66) was used to remove, 
dereplicate, and denoise reads shorter than $380 \mathrm{bp}$, leading to zero-radius operational taxonomic units (zOTUs). Furthermore, chimeric sequences were removed. For this purpose, UCHIME2 was used in reference mode against the SILVA SSU database version 128 (67). To create a zOTU table, the qualityfiltered sequences were mapped to chimera-free OTUs using USEARCH. With parallel_assign_taxonomy_blast.py taxonomic classification of the zOTU sequences against the SILVA database was done. Filter_otu_table.py was used for removal of chloroplasts, unclassified OTUs, and extrinsic domain OTUs. Finally, the lowest number of sequences by random subsampling $(12,100$ reads per sample) was used for sample comparison at the same surveying effort.

Metagenome sequencing and analysis. DNA isolated from each culture triplicate was pooled in equal amounts. Paired-end metagenome sequencing was performed with an Illumina HiSeq 4000 $(2 \times 150 \mathrm{bp})$ using the Nextera XT DNA library prep kit. For quality-trimming and verification of the paired-end reads, Trimmomatic version $0.36(68)$ and FastQC version $0.11 .5(69)$ were used. Assembly and coverage calculation was performed with SPAdes version 3.10 (70). Contigs >200 bp were annotated with Prokka 1.11 (71). For taxonomic assignment of nitrilases, a BLASTn search (72) of the nitrile containing contigs was performed against the NCBI non-redundant database. Abundance of nitrile-degrading genes was calculated by mapping quality-filtered reads against the sequence-based identified putative nitrile-converting genes with Bowtie2 version 2.3.4.2 (73). Normalization was done by dividing the number of mapped nucleotides by the total number of nucleotides. Subsequently, these values were normalized against the number of putative target genes.

Phylogenetic tree. Evolutionary analyses were conducted in MEGA X version 10.0.5 (74). Alignment of the amino acid sequences was done with MUSCLE (75) and standard settings. The evolutionary history was inferred by using the Maximum Likelihood method and LG model (76) as recommended by buildin model test tool of MEGA X. The tree with the highest log likelihood (-31012.19) is shown. Initial tree(s) for the heuristic search were obtained automatically by applying Neighbor-Join and BioNJ algorithms to a matrix of pairwise distances estimated using a JTT model, and then selecting the topology with superior log likelihood value. A discrete Gamma distribution was used to model evolutionary rate differences among sites ( 5 categories $(+G$, parameter $=1.1643)$ ). This analysis involved 276 amino acid sequences. A total of 491 positions were in the final dataset. Extensive subtree pruning regrafting and a very weak branch swap filter were used. Phylogeny was tested by calculating 500 bootstrap replicates and nodes with values below $50 \%$ were condensed for the final image. Reference sequences were recovered from the NCBI database (accession numbers in parentheses): For aromatic nitrilases, Rhodococcus rhodochrous J1 (BAA01994) (77), R. ruber NHB-2 (CCN27135) (78), and Gordonia terrae MTCC 8139 (AGR86048) (79) were used as references. Aliphatic nitrilases were derived from Comamonas testosteroni (AAA82085) (80), Synechocystis sp. PCC6803 (BAA10717) (81), 
and $R$. rhodochrous K22 (BAA02127) (82), while arylacetonitrilases were obtained from Alcaligenes faecalis JM3 (BAA02684) (83), Pseudomonas fluorescens EBC191 (AAW79573) (84), and Pseudomonas putida MTCC 5110 (ABV21758) (85). $\beta$-Cyano-L-alanine nitrilases were derived from $P$. fluorescens PfO1 (ABA74312) (86, 87), Pseudomonas protegens Pf-5 (AAY92181) (86, 88), and Pseudomonas pseudoalcaligenes CECT 5344 (CDM40486) (89, 90). Cyanide dihydratases were retrieved from Pseudomonas stutzeri AK61 (AIY29195) (91), Bacillus pumilus C1 (AF392815) (92), and B. pumilus 8A3 (AAN77003) (92). Furthermore, three amidases were included, originating from Pseudomonas aeruginosa PAC142 (P11436) (93), R. rhodochrous M8 (AAX83004) (94), and Geobacillus pallidus RAPc8 (AAO23013) (95).

Accession numbers. Raw data of 16S rRNA gene analysis and metagenome sequences as well as assembled metagenomes have been deposited at NCBI under the following biosample accession numbers: compost sample, SAMN09932763; filtered compost sample, SAMN09932764; control after 68 h, SAMN09932785; control after 188 h, SAMN09932808; control after 166 h, SAMN09932809; control after 214 h, SAMN09932848; phenylacetonitrile enrichment, SAMN09930795; succinonitrile enrichment, SAMN09932325; acetonitrile enrichment, SAMN09932597; crotononitrile enrichment; SAMN09932603; 4-hydroxybenzonitrile enrichment, SAMN09932608; cyclohexanecarbonitrile enrichment, SAMN09932617; phenylacetic acid enrichment, SAMN09932620; succinic acid enrichment, SAMN09932700; acetic acid enrichment, SAMN09932745; crotonic acid enrichment, SAMN09932756; 4-hydroxybenzoic acid enrichment, SAMN09932757; cyclohexanecarboxylic acid enrichment, SAMN09932758.

\section{ACKNOWLEDGMENT}

The work of R.E. was supported by the "Fonds der Chemischen Industrie im Verband der Chemischen Industrie e.V.". The funders had no role in study design, data collection, and interpretation, or the decision to submit the work for publication. 


\section{REFERENCES}

1. Mahadevan S. 1973. Role of oximes in nitrogen metabolism in plants. Annu Rev Plant Physiol 24:69-88.

2. Legras JL, Chuzel G, Arnaud A, Galzy P. 1990. Natural nitriles and their metabolism. World J Microbiol Biotechnol 6:83-108.

3. Scheuer PJ. 1992. Isocyanides and cyanides as natural products. Acc Chem Res 25:433-439.

4. Fleming FF. 1999. Nitrile-containing natural products. Nat Prod Rep 16:597-606.

5. Conn EE. 1969. Cyanogenic glycosides. J Agric Food Chem 17:519-526.

6. Eyjólfsson R. 1970. Recent advances in the chemistry of cyanogenic glycosides, p. 74-108. In Herz, W, Grisebach, H, Scott, Al (eds.), Fortschritte der Chemie organischer Naturstoffe / Progress in the chemistry of organic natural products, 1st ed. Springer Vienna, Vienna, Austria.

7. Poulton JE. 1990. Cyanogenesis in plants. Plant Physiol 94:401-405.

8. Tanii H, Hashimoto K. 1984. Studies on the mechanism of acute toxicity of nitriles in mice. Arch Toxicol 55:47-54.

9. Grogan J, DeVito SC, Pearlman RS, Korzekwa KR. 1992. Modeling cyanide release from nitriles: Prediction of cytochrome P450 mediated acute nitrile toxicity. Chem Res Toxicol 5:548-552.

10. Uetrecht JP, Trager W. 2007. Oxidation pathways and the enzymes that mediate them, p. 33108. In Uetrecht, JP, Trager, W (eds.), Drug metabolism: Chemical and enzymatic aspects, 1st ed. Informa Healthcare, New York, NY, USA.

11. Willhite CC, Smith RP. 1981. The role of cyanide liberation in the acute toxicity of aliphatic nitriles. Toxicol Appl Pharmacol 59:589-602.

12. Lewis DFV, Wiseman A. 2005. A selective review of bacterial forms of cytochrome P450 enzymes. Enzyme Microb Technol 36:377-384.

13. Richter O-MH, Ludwig B. 2003. Cytochrome c oxidase - structure, function, and physiology of a redox-driven molecular machine, p. 47-74. In Amara, SG, Bamberg, E, Blaustein, MP, Grunicke, H, Jahn, R, Lederer, WJ, Miyajima, A, Murer, H, Offermanns, S, Pfanner, N, Schultz, G, Schweiger, $M$ (eds.), Reviews of physiology, biochemistry and pharmacology, 1st ed. Springer Berlin Heidelberg, Berlin, Germany.

14. Pace HC, Brenner C. 2001. The nitrilase superfamily: classification, structure and function. Genome Biol 2:reviews0001.1.

15. Vergne-Vaxelaire C, Bordier F, Fossey A, Besnard-Gonnet M, Debard A, Mariage A, Pellouin V, Perret A, Petit J-L, Stam M, Salanoubat M, Weissenbach J, De Berardinis V, Zaparucha A. 2013. Nitrilase activity screening on structurally diverse substrates: providing biocatalytic tools for organic synthesis. Adv Synth Catal 355:1763-1779.

16. Ramteke PW, Maurice NG, Joseph B, Wadher BJ. 2013. Nitrile-converting enzymes: an ecofriendly tool for industrial biocatalysis. Biotechnol Appl Biochem 60:459-481.

17. Kurenkov VF. 1997. Acrylamide polymers, p. 61-72. In Cheremisinoff, NP (ed.), Handbook of engineering polymeric materials, 1st ed. Marcel Dekker, New York, NY, USA. 
18. Fleming FF, Yao L, Ravikumar PC, Funk L, Shook BC. 2010. Nitrile-containing pharmaceuticals: Efficacious roles of the nitrile pharmacophore. J Med Chem 53:7902-7917.

19. Holtze MS, Hansen HCB, Juhler RK, Sørensen J, Aamand J. 2007. Microbial degradation pathways of the herbicide dichlobenil in soils with different history of dichlobenil-exposure. Environ Pollut 148:343-351.

20. Baxter J, Cummings SP. 2008. The degradation of the herbicide bromoxynil and its impact on bacterial diversity in a top soil. J Appl Microbiol 104:1605-1616.

21. Tanner AC. 1985. The role of bacteria in the cycling of nutrients within the maritime antarctic environment, p. 123-127. In Siegfried, WR, Condy, PR, Laws, RM (eds.), Antarctic nutrient cycles and food webs, 1st ed. Springer Berlin Heidelberg, Berlin, Germany.

22. Alongi DM. 1994. The role of bacteria in nutrient recycling in tropical mangrove and other coastal benthic ecosystems. Hydrobiologia 285:19-32.

23. Rashid MI, Mujawar LH, Shahzad T, Almeelbi T, Ismail IMI, Oves M. 2016. Bacteria and fungi can contribute to nutrients bioavailability and aggregate formation in degraded soils. Microbiol Res 183:26-41.

24. Ahmed AE, Farooqui MY. 1982. Comparative toxicities of aliphatic nitriles. Toxicol Lett 12:157163.

25. Wallig MA, Gould DH, Fettman MJ, Willhite CC. 1988. Comparative toxicities of the naturally occurring nitrile 1-cyano-3,4-epithiobutane and the synthetic nitrile n-valeronitrile in rats: Differences in target organs, metabolism and toxic mechanisms. Food Chem Toxicol 26:149157.

26. Jorgensen JH, Ferraro MJ. 2009. Antimicrobial susceptibility testing: A review of general principles and contemporary practices. Clin Infect Dis 49:1749-1755.

27. Marhan J. 1995. Mutagenicity of cytostatic drugs in a bacterial system. I. Ames test. Folia Microbiol (Praha) 40:457-461.

28. Bending GD, Rodríguez-Cruz MS, Lincoln SD. 2007. Fungicide impacts on microbial communities in soils with contrasting management histories. Chemosphere 69:82-88.

29. Malathi VM, Jalali SK, Lyju VJ, Gracy RG, More RP, Anandham R, Thulasi A, Venkatesan T. 2017. Associated bacterial diversity of insecticide-susceptible and -resistant brown planthopper, Nilaparvata lugens (Homoptera: Delphacidae) analyzed by culture-dependent and independent methods. Phytoparasitica 45:683-693.

30. Abo-El-Dahab M, El-Goorani M, El-Wakil M. 1977. Effect of certain chemicals on the vitro growth of Agrobacterium tumefaciens and on the gall formation on artificially infected plants. Egypt J Phytophatol 9:43-57.

31. Holly K. 1964. Ioxynil and bromoxynil as herbicides. Int J Pest Manag Part C 10:256-258.

32. Holtze MS, Sørensen SR, Sørensen J, Aamand J. 2008. Microbial degradation of the benzonitrile herbicides dichlobenil, bromoxynil and ioxynil in soil and subsurface environments - Insights into degradation pathways, persistent metabolites and involved degrader organisms. Environ Pollut 154:155-168. 
33. Abbas Z, Akmal M, Khan KS, Fayyaz-ul-Hassan. 2014. Effect of buctril super (Bromoxynil) herbicide on soil microbial biomass and bacterial population. Brazilian Arch Biol Technol 57:914.

34. Egelkamp R, Schneider D, Hertel R, Daniel R. 2017. Nitrile-degrading bacteria isolated from compost. Front Environ Sci 5:56.

35. Tanii H. 2017. Allyl nitrile: Toxicity and health effects. J Occup Health 59:104-111.

36. Yerabolu JR, Liotta CL, Krishnamurthy R. 2017. Anchimeric-assisted spontaneous hydrolysis of cyanohydrins under ambient conditions: Implications for cyanide-initiated selective transformations. Chem - A Eur J 23:8756-8765.

37. Gerasimova T, Novikov A, Osswald S, Yanenko A. 2004. Screening, characterization and application of cyanide-resistant nitrile hydratases. Eng Life Sci 4:543-546.

38. Bhatia SK, Mehta PK, Bhatia RK, Bhalla TC. 2013. An isobutyronitrile-induced bienzymatic system of Alcaligenes sp. MTCC 10674 and its application in the synthesis of $\alpha$ hydroxyisobutyric acid. Bioprocess Biosyst Eng 36:613-625.

39. Bauer R, Knackmuss H-J, Stolz A. 1998. Enantioselective hydration of 2-arylpropionitriles by a nitrile hydratase from Agrobacterium tumefaciens strain d3. Appl Microbiol Biotechnol 49:8995.

40. Nagasawa T, Mauger J, Yamada H. 1990. A novel nitrilase, arylacetonitrilase, of Alcaligenes faecalis JM3. Purification and characterization. Eur J Biochem 194:765-772.

41. Schreiner U, Hecher B, Obrowsky S, Waich K, Klempier N, Steinkellner G, Gruber K, Rozzell JD, Glieder A, Winkler M. 2010. Directed evolution of Alcaligenes faecalis nitrilase. Enzyme Microb Technol 47:140-146.

42. Layh N, Hirrlinger B, Stolz A, Knackmuss H-J. 1997. Enrichment strategies for nitrile-hydrolysing bacteria. Appl Microbiol Biotechnol 47:668-674.

43. Layh N, Parratt J, Willetts A. 1998. Characterization and partial purification of an enantioselective arylacetonitrilase from Pseudomonas fluorescens DSM 7155. J Mol Catal B Enzym 5:467-474.

44. Gilligan T, Yamada H, Nagasawa T. 1993. Production of S-(+)-2-phenylpropionic acid from (R,S)2-phenylpropionitrile by the combination of nitrile hydratase and stereoselective amidase in Rhodococcus equi TG328. Appl Microbiol Biotechnol 39:720-725.

45. Torsvik V, Sørheim R, Goksøyr J. 1996. Total bacterial diversity in soil and sediment communities - a review. J Ind Microbiol Biotechnol 17:170-178.

46. Davis KER, Joseph SJ, Janssen PH. 2005. Effects of growth medium, inoculum size, and incubation time on culturability and isolation of soil bacteria. Appl Environ Microbiol 71:826834.

47. Solden L, Lloyd K, Wrighton K. 2016. The bright side of microbial dark matter: Lessons learned from the uncultivated majority. Curr Opin Microbiol 31:217-226.

48. Benedik MJ, Sewell BT. 2018. Cyanide-degrading nitrilases in nature. J Gen Appl Microbiol 64:90-93. 
49. Jin L-Q, Liu Z-Q, Zheng Y-G, Shen Y-C. 2010. Identification and characterization of Serratia marcescens ZJB-09104, a nitrile-converting bacterium. World J Microbiol Biotechnol 26:817823.

50. Santoshkumar M, Nayak AS, Anjaneya O, Karegoudar TB. 2010. A plate method for screening of bacteria capable of degrading aliphatic nitriles. J Ind Microbiol Biotechnol 37:111-115.

51. Finnegan I, Toerien S, Abbot L, Smit F, Raubenheimer HG. 1991. Identification and characterisation of an Acinetobacter sp. capable of assimilation of a range of cyano-metal complexes, free cyanide ions and simple organic nitriles. Appl Microbiol Biotechnol 36:142144.

52. Yamamoto K, Ueno Y, Otsubo K, Kawakami K, Komatsu K. 1990. Production of S-(+)-ibuprofen from a nitrile compound by Acinetobacter sp. strain AK226. Appl Environ Microbiol 56:31253129.

53. Viana AT, Caetano T, Covas C, Santos T, Mendo S. 2018. Environmental superbugs: The case study of Pedobacter spp. Environ Pollut 241:1048-1055.

54. Tipayno SC, Truu J, Samaddar S, Truu M, Preem J-K, Oopkaup K, Espenberg M, Chatterjee P, Kang Y, Kim K, Sa T. 2018. The bacterial community structure and functional profile in the heavy metal contaminated paddy soils, surrounding a nonferrous smelter in South Korea. Ecol Evol 8:6157-6168.

55. Romaniuk K, Ciok A, Decewicz P, Uhrynowski W, Budzik K, Nieckarz M, Pawlowska J, Zdanowski MK, Bartosik D, Dziewit L. 2018. Insight into heavy metal resistome of soil psychrotolerant bacteria originating from King George Island (Antarctica). Polar Biol 41:1319-1333.

56. Grant WN, Stasiuk S, Newton-Howes J, Ralston M, Bisset SA, Heath DD, Shoemaker CB. 2006. Parastrongyloides trichosuri, a nematode parasite of mammals that is uniquely suited to genetic analysis. Int J Parasitol 36:453-466.

57. Kobayashi M, Yanaka N, Nagasawa T, Yamada H. 1990. Purification and characterization of a novel nitrilase of Rhodococcus rhodochrous K22 that acts on aliphatic nitriles. J Bacteriol 172:4807-4815.

58. Miller JH. 1972. Experiments in molecular genetics, 1st ed. Cold Spring Harbor Laboratory, Cold Spring Harbor, NY, USA.

59. Swiderska A, Berndtson AK, Cha MR, Li L, Beaudoin GM, Zhu J, Fuqua C. 2001. Inhibition of the Agrobacterium tumefaciens TraR quorum-sensing regulator. Interactions with the TraM antiactivator. J Biol Chem 276:49449-49458.

60. Cohn F. 1872. Untersuchungen über Bacterien, p. 127-224. In Beiträge zur Biologie der Pflanzen, 1st ed. J. U. Kern's Verlag, Breslau, Poland.

61. Kinoshita S, Nakayama K, Akita S. 1958. Taxonomical study of glutamic acid accumulating bacteria, Micrococcus glutamicus nov. sp. Bull Agric Chem Soc Japan 22:176-185.

62. Klindworth A, Pruesse E, Schweer T, Peplies J, Quast C, Horn M, Glöckner FO. 2013. Evaluation of general $16 \mathrm{~S}$ ribosomal RNA gene PCR primers for classical and next-generation sequencingbased diversity studies. Nucleic Acids Res 41:e1. 
63. Caporaso JG, Kuczynski J, Stombaugh J, Bittinger K, Bushman FD, Costello EK, Fierer N, Peña AG, Goodrich JK, Gordon JI, Huttley GA, Kelley ST, Knights D, Koenig JE, Ley RE, Lozupone CA, McDonald D, Muegge BD, Pirrung M, Reeder J, Sevinsky JR, Turnbaugh PJ, Walters WA, Widmann J, Yatsunenko T, Zaneveld J, Knight R. 2010. QIIME allows analysis of high-throughput community sequencing data. Nat Methods 7:335-336.

64. Zhang J, Kobert K, Flouri T, Stamatakis A. 2014. PEAR: a fast and accurate Illumina Paired-End reAd mergeR. Bioinformatics 30:614-620.

65. Martin M. 2011. Cutadapt removes adapter sequences from high-throughput sequencing reads. EMBnet.journal 17:10.

66. Edgar RC. 2010. Search and clustering orders of magnitude faster than BLAST. Bioinformatics 26:2460-2461.

67. Yilmaz P, Parfrey LW, Yarza P, Gerken J, Pruesse E, Quast C, Schweer T, Peplies J, Ludwig W, Glöckner FO. 2014. The SILVA and "All-species Living Tree Project (LTP)" taxonomic frameworks. Nucleic Acids Res 42:D643-D648.

68. Bolger AM, Lohse M, Usadel B. 2014. Trimmomatic: a flexible trimmer for Illumina sequence data. Bioinformatics 30:2114-2120.

69. Andrews S. 2010. FastQC: a quality control tool for high throughput sequence data. https://www.bioinformatics.babraham.ac.uk/projects/fastqc/

70. Bankevich A, Nurk S, Antipov D, Gurevich AA, Dvorkin M, Kulikov AS, Lesin VM, Nikolenko SI, Pham S, Prjibelski AD, Pyshkin A V, Sirotkin A V, Vyahhi N, Tesler G, Alekseyev MA, Pevzner PA. 2012. SPAdes: a new genome assembly algorithm and its applications to single-cell sequencing. J Comput Biol 19:455-477.

71. Seemann T. 2014. Prokka: rapid prokaryotic genome annotation. Bioinformatics 30:2068-2069.

72. Altschul SF, Gish W, Miller W, Myers EW, Lipman DJ. 1990. Basic local alignment search tool. J Mol Biol 215:403-410.

73. Langmead B, Salzberg SL. 2012. Fast gapped-read alignment with Bowtie 2. Nat Methods 9:357359.

74. Kumar S, Stecher G, Li M, Knyaz C, Tamura K. 2018. MEGA X: Molecular Evolutionary Genetics Analysis across computing platforms. Mol Biol Evol 35:1547-1549.

75. Edgar RC. 2004. MUSCLE: multiple sequence alignment with high accuracy and high throughput. Nucleic Acids Res 32:1792-1797.

76. Le SQ, Gascuel O. 2008. An improved general amino acid replacement matrix. Mol Biol Evol 25:1307-1320.

77. Kobayashi M, Komeda H, Yanaka N, Nagasawa T, Yamada H. 1992. Nitrilase from Rhodococcus rhodochrous J1: Sequencing and overexpression of the gene and identification of an essential cysteine residue. J Biol Chem 267:20746-20751.

78. Kaplan $\mathrm{O}$, Veselá $A B$, Petříčková A, Pasquarelli F, Pičmanová M, Rinágelová A, Bhalla TC, Pátek M, Martínková L. 2013. A comparative study of nitrilases identified by genome mining. Mol Biotechnol 54:996-1003. 
79. Kumar V, Seth A, Kumari V, Kumar V, Bhalla TC. 2015. Purification, characterization and in-silico analysis of nitrilase from Gordonia terrae. Protein Pept Lett 22:52-62.

80. Lévy-Schil S, Soubrier F, Crutz-Le Coq AM, Faucher D, Crouzet J, Pétré D. 1995. Aliphatic nitrilase from a soil-isolated Comamonas testosteroni sp.: Gene cloning and overexpression, purification and primary structure. Gene 161:15-20.

81. Heinemann U, Engels D, Bürger S, Kiziak C, Mattes R, Stolz A. 2003. Cloning of a nitrilase gene from the cyanobacterium Synechocystis sp. strain PCC6803 and heterologous expression and characterization of the encoded protein. Appl Environ Microbiol 69:4359-4366.

82. Kobayashi M, Yanaka N, Nagasawa T, Yamada H. 1992. Primary structure of an aliphatic nitriledegrading enzyme, aliphatic nitrilase, from Rhodococcus rhodochrous $\mathrm{K} 22$ and expression of its gene and identification of its active site residue. Biochemistry 31:9000-9007.

83. Kobayashi M, Izui H, Nagasawa T, Yamada H. 1993. Nitrilase in biosynthesis of the plant hormone indole-3-acetic acid from indole-3-acetonitrile: cloning of the Alcaligenes gene and site-directed mutagenesis of cysteine residues. Proc Natl Acad Sci U S A 90:247-251.

84. Kiziak C, Conradt D, Stolz A, Mattes R, Klein J. 2005. Nitrilase from Pseudomonas fluorescens EBC191: Cloning and heterologous expression of the gene and biochemical characterization of the recombinant enzyme. Microbiology 151:3639-3648.

85. Banerjee A, Dubey S, Kaul P, Barse B, Piotrowski M, Banerjee UC. 2009. Enantioselective nitrilase from Pseudomonas putida: Cloning, heterologous expression, and bioreactor studies. Mol Biotechnol 41:35-41.

86. Howden AJM, Harrison CJ, Preston GM. 2009. A conserved mechanism for nitrile metabolism in bacteria and plants. Plant J 57:243-253.

87. Silby MW, Cerdeño-Tárraga AM, Vernikos GS, Giddens SR, Jackson RW, Preston GM, Zhang X-X, Moon CD, Gehrig SM, Godfrey SAC, Knight CG, Malone JG, Robinson Z, Spiers AJ, Harris S, Challis GL, Yaxley AM, Harris D, Seeger K, Murphy L, Rutter S, Squares R, Quail MA, Saunders E, Mavromatis K, Brettin TS, Bentley SD, Hothersall J, Stephens E, Thomas CM, Parkhill J, Levy SB, Rainey PB, Thomson NR. 2009. Genomic and genetic analyses of diversity and plant interactions of Pseudomonas fluorescens. Genome Biol 10:R51.

88. Paulsen IT, Press CM, Ravel J, Kobayashi DY, Myers GSA, Mavrodi D V., DeBoy RT, Seshadri R, Ren Q, Madupu R, Dodson RJ, Durkin AS, Brinkac LM, Daugherty SC, Sullivan SA, Rosovitz MJ, Gwinn ML, Zhou L, Schneider DJ, Cartinhour SW, Nelson WC, Weidman J, Watkins K, Tran K, Khouri H, Pierson EA, Pierson LS, Thomashow LS, Loper JE. 2005. Complete genome sequence of the plant commensal Pseudomonas fluorescens Pf-5. Nat Biotechnol 23:873-878.

89. Acera F, Carmona MI, Castillo F, Quesada A, Blasco R. 2017. A cyanide-induced 3-cyanoalanine nitrilase in the cyanide-assimilating bacterium Pseudomonas pseudoalcaligenes strain CECT 5344. Appl Environ Microbiol 83:1-14.

90. Wibberg $D$, Luque-Almagro VM, Igeño MI, Bremges A, Roldán MD, Merchán F, Sáez LP, Guijo MI, Manso MI, Macías D, Cabello P, Becerra G, Ibáñez MI, Carmona MI, Escribano MMP, Castillo F, Sczyrba A, Moreno-Vivián C, Blasco R, Pühler A, Schlüter A. 2014. Complete genome sequence of the cyanide-degrading bacterium Pseudomonas pseudoalcaligenes CECT5344. J Biotechnol 175:67-68. 
91. Crum MA-N, Park JM, Mulelu AE, Sewell BT, Benedik MJ. 2015. Probing C-terminal interactions of the Pseudomonas stutzeri cyanide-degrading CynD protein. Appl Microbiol Biotechnol 99:3093-102.

92. Jandhyala D, Berman M, Meyers PR, Sewell BT, Willson RC, Benedik MJ. 2003. CynD, the cyanide dihydratase from Bacillus pumilus: gene cloning and structural studies. Appl Environ Microbiol 69:4794-4805.

93. Ambler RP, Auffret AD, Clarke PH. 1987. The amino acid sequence of the aliphatic amidase from Pseudomonas aeruginosa. FEBS Lett 215:285-290.

94. Riabchenko LE, Podcherniaev DA, Kotlova EK, Yanenko AS. 2006. Cloning the amidase gene from Rhodococcus rhodochrous M8 and its expression in Escherichia coli. Genetika 42:1075-1082.

95. Pereira RA, Graham D, Rainey FA, Cowan DA. 1998. A novel thermostable nitrile hydratase. Extremophiles 2:347-357. 


\subsection{Nitrile-degrading bacteria isolated from compost}

Richard Egelkamp ${ }^{1}$, Dominik Schneider ${ }^{1}$, Robert Hertel ${ }^{1} \&$ Rolf Daniel $^{1}$

Frontiers in Environmental Science (2017), 5: 56

(doi: 10.3389/fenvs.2017.00056)

${ }^{1}$ Genomic and Applied Microbiology \& Göttingen Genomics Laboratory, Institute of Microbiology and Genetics, University of Göttingen, Göttingen, Germany

Author contributions

Performed the experiments: $\mathbf{R E}$

Analyzed the data: RE, DS

Wrote the publication: $\mathrm{RE}, \mathrm{DS}, \mathrm{RH}, \mathrm{RD}$

Conceived the study: $\mathrm{RH}, \mathrm{RD}$ 


\title{
Nitrile-Degrading Bacteria Isolated from Compost
}

\author{
Richard Egelkamp, Dominik Schneider, Robert Hertel and Rolf Daniel * \\ Genomic and Applied Microbiology, Göttingen Genomics Laboratory, Institute of Microbiology and Genetics, University of \\ Goettingen, Göttingen, Germany
}

Keywords: acetonitrile, nitrilase, nitrile hydratase, amidase, Flavobacterium, Pseudomonas, Rhodococcus, Variovorax

\section{INTRODUCTION}

Nitriles are a diverse group of organic compounds with $-\mathrm{C} \equiv \mathrm{N}$ as functional group. Most nitriles are slightly cytotoxic but some cause severe toxic effects. More than 120 naturally occurring nitriles without considering cyanogenic glycosides are present in terrestrial and marine habitats, especially in plant components such as almonds or other fruit pits. The most common group of naturally occurring nitriles are cyanogenic glycosides, which can be found in more than 100 plant families as well as in fungi, bacteria, and animals. This group of molecules can be chemically or enzymatically hydrolyzed, leading to the release of highly toxic hydrogen cyanide and thereby act as natural defense compound (Fleming, 1999). For detoxification, two enzymatic pathways for the degradation of nitriles are known. The first one involves nitrilases (EC 3.5.5.1), a subgroup of the carbon-nitrogen hydrolase superfamily, which degrade nitriles directly to carboxylic acids and ammonia. The second one is a bi-enzymatic pathway using nitrile hydratases (NHases; EC 4.2.1.84) for the degradation of nitriles to amides and amidases (EC 3.5.1.4) for the subsequent degradation to carboxylic acids and ammonia (Gong et al., 2012). The enzymatic hydrolysis of nitriles proceeds under mild reaction conditions, whereas the chemical hydrolysis is dependent on acidic or alkaline conditions and high temperatures. The latter also results in the production of large quantities of byproducts and inorganic waste (Clouthier and Pelletier, 2012; Vergne-Vaxelaire et al., 2013). Consequently, nitrile-converting enzymes are of increasing industrial importance with respect to green chemistry. A constantly increasing number of nitrile-derived amides [e.g., acrylamides or carboxylic acids (e.g., glycolic acid)] are produced with these enzymes (Schmid et al., 2001; Panova et al., 2007). In addition, nitrilases can be used for the treatment of nitrile-polluted wastewater ( $\mathrm{Li}$ et al., 2016) and other environmentally-friendly bioremediation processes (Gong et al., 2012).

Here, we report data on the taxonomic composition of an enrichment culture with acetonitrile as nitrogen source. In addition, we present eight individual bacterial draft genome sequences of isolates obtained from this enrichment. The genome content of these isolates was analyzed with respect to genes responsible for the nitrile-degrading phenotype. Genome and average nucleotide identity analysis indicated that the isolated bacterial strains are affiliated to the species Rhodococcus erythropolis, Flavobacterium sp., Variovorax boronicumulans, Pseudomonas sp., and Pseudomonas kilonensis.

\section{MATERIALS AND METHODS}

\section{Isolation of the Bacteria}

Compost (100 g, pH 7.5) of the Experimental Botanical Garden Göttingen, Germany ( $51^{\circ} 33^{\prime} 22.6^{\prime \prime} \mathrm{N}$ $9^{\circ} 57^{\prime} 16.2^{\prime \prime} \mathrm{E}$ ) was suspended in $500 \mathrm{ml} \mathrm{H}_{2} \mathrm{O}$ and filtered with a $2.7 \mu \mathrm{m} \mathrm{GF} / \mathrm{D}$ glass fiber filter (Whatman, Little Chalfont, UK). Enrichment and control cultures were each initiated with $750 \mu \mathrm{l}$ of the resulting filtrate. Enrichment cultures were grown in $40 \mathrm{ml} \mathrm{M} 9$ medium 
(Atlas, 2010) with ATCC trace mineral supplement (LGC Standards, Teddington, UK), $10 \mathrm{mM}$ glucose as carbon source and $25 \mathrm{mM}$ acetonitrile as sole nitrogen source at $25^{\circ} \mathrm{C}$ for 4 days. Control cultures were grown in same medium but the medium contained $18.7 \mathrm{mM} \mathrm{NH}_{4} \mathrm{Cl}$ instead of acetonitrile. The enrichment culture was streaked on solid M9 medium with $25 \mathrm{mM}$ acetonitrile as sole nitrogen source and incubated at $25^{\circ} \mathrm{C}$. The obtained 33 colonies showed four distinct colony morphologies, and were picked and purified over five rounds of incubation. Growth in the absence of an added nitrogen source was not recorded.

\section{$16 S$ rRNA Gene Sequencing}

Genomic DNA of the liquid cultures and the specific isolates was extracted using the MasterPure complete DNA and RNA purification kit (Epicentre, Madison, WI, USA), while DNA isolation of the compost sample was done with the PowerMax soil DNA isolation kit (MO BIO Laboratories, Carlsbad, CA, USA). The bacterial composition was determined by amplicon-based analysis of the V3-V4 region of the $16 \mathrm{~S}$ rRNA gene using the bacterial primers S-D-Bact-0341-b-S-17 and S-D-Bact-0785-a-A21 (Klindworth et al., 2013) with adapters for Illumina MiSeq sequencing (Illumina, San Diego, CA, USA). The PCR reaction mixture $(50 \mu \mathrm{l})$ contained $10 \mu \mathrm{l} 5$-fold Phusion GC buffer, $200 \mu \mathrm{M}$ of each of the four dNTPs, $2.5 \mu \mathrm{l}$ DMSO, $0.2 \mu \mathrm{M}$ of each primer, $200 \mu \mathrm{M} \mathrm{MgCl}_{2}, 1 \mathrm{U}$ of Phusion polymerase (Thermo Fisher Scientific, Waltham, MA, USA), and $25 \mathrm{ng}$ of isolated DNA as template. The following cycling scheme was used: initial denaturation at $98^{\circ} \mathrm{C}$ for $1 \mathrm{~min}$ and 25 cycles of denaturation at $98^{\circ} \mathrm{C}$ for $45 \mathrm{~s}$, annealing at $60^{\circ} \mathrm{C}$ for $45 \mathrm{~s}$, and extension at $72^{\circ} \mathrm{C}$ for $30 \mathrm{~s}$, followed by a final extension at $72^{\circ} \mathrm{C}$ for $5 \mathrm{~min}$. PCR reactions were performed in triplicate for each sample. The resulting PCR products were pooled in equal amounts and purified using the NucleoMag 96 PCR kit (MachereyNagel, Düren, Germany) as recommended by the manufacturer. Quantification of the PCR products was performed using the Quant-iT dsDNA HS assay kit and a Qubit fluorometer as recommended by the manufacturer (Invitrogen, Carlsbad, CA, USA). Indexing of the PCR products was performed with Nextera XT DNA library prep kit as described by the supplier (Illumina). Sequencing was performed with the Illumina MiSeq platform using the dual index paired-end approach $(2 \times 300 \mathrm{bp})$ and v3 chemistry.

The 16S rRNA genes of specific isolates were amplified with the primer pair 08f ( $5^{\prime}$-AGAGTTTGATCCTGGC- $\left.3^{\prime}\right)$ and $1504 \mathrm{r}\left(5^{\prime}\right.$-TACCTTGTTACGACTT-3'). The previously mentioned cycling scheme was modified to an annealing temperature of $40^{\circ} \mathrm{C}$ and an extension time of $45 \mathrm{~s}$. Sanger sequencing of the PCR products was done by Seqlab (Göttingen, Germany).

\section{S rRNA Gene Amplicon Analysis}

Demultiplexing and clipping of sequence adapters from raw amplicon sequences were performed by employing CASAVA data analysis software (Illumina). Paired-end sequences were merged using PEAR v0.9.10 with default parameters (Zhang et al., 2014). Subsequently, sequences with an average quality score lower than 20 and containing unresolved bases were removed with the split_libraries_fastq.py script of QIIME 1.9.1 (Caporaso et al., 2010). Non-clipped reverse and forward primer sequences were removed by employing cutadapt 1.12 with default settings (Martin, 2011). USEARCH version 9.2.64 was used following the UNOISE pipeline (Edgar, 2010). In detail, reads shorter than 380 bp were removed, dereplicated, and denoised with the UNOISE2 algorithm of USEARCH resulting in zero-radius operational taxonomic units (zOTUs). Additionally, chimeric sequences were removed using UCHIME2 in reference mode against the SILVA SSU database release 128 (Yilmaz et al., 2014). All quality-filtered sequences were mapped to chimera-free OTUs and a zOTU table was created using USEARCH. Taxonomic classification of the picked reference sequences (zOTUs) was performed with parallel_assign_taxonomy_blast.py against the same SILVA database. Extrinsic domain OTUs, chloroplasts, and unclassified OTUs were removed from the dataset by employing filter_otu_table.py. Sample comparisons were performed at same surveying effort, utilizing the lowest number of sequences by random subsampling (31,000 reads per sample).

\section{Genome Sequencing and Analysis}

Genome sequencing was performed using an Illumina MiSeq system with the Nextera XT DNA library prep kit as recommended by the manufacturer (Illumina). Paired-end reads were quality-trimmed with Trimmomatic version 0.36 (Bolger et al., 2014) and verified with FastQC version 0.11.5 (Andrews, 2010). Assembly and first coverage calculation was performed with SPAdes version 3.9.0 (Bankevich et al., 2012). All contigs $>500$ bp and with a coverage $>5$ were annotated using Prokka version 1.11 (Seemann, 2014). Final coverage was calculated with Bowtie 2 version 2.2.9 (Langmead and Salzberg, 2012). A BLASTn search (Altschul et al., 1990) was performed against the NCBI non-redundant database to verify assembled contigs. For taxonomic assignment, the full-length $16 \mathrm{~S}$ rRNA gene sequences of all isolated organisms were searched against the NCBI database. Combination of these data with an analysis of the tetra-nucleotide signatures (Tetra) and the average nucleotide identities (ANI) performed with JSpeciesWS (Richter et al., 2016) as well as in silico DNA-DNA hybridization results calculated with the online tool GGDC 2.1 (Meier-Kolthoff et al., 2013) were employed for taxonomic classification of the isolates. Putative nitrilases, NHases, and amidases were compared with the nonredundant protein database of the NCBI using BLASTp (Altschul et al., 1990). The hit with the highest score is given in Table 1.

\section{RESULTS AND DISCUSSION}

Enrichment of the compost sample with M9 minimal medium containing acetonitrile as sole nitrogen source resulted in microbial growth, indicating the presence of acetonitriledegrading organisms. The bacterial diversity and composition of the compost sample and the enrichment culture were determined via amplicon-based 16S rRNA gene analysis. The compost sample used as starting material for enrichment comprised 520 zOTUs, which were reduced to 63 during enrichment (Supplementary Table S1). The dominant bacterial genera after 


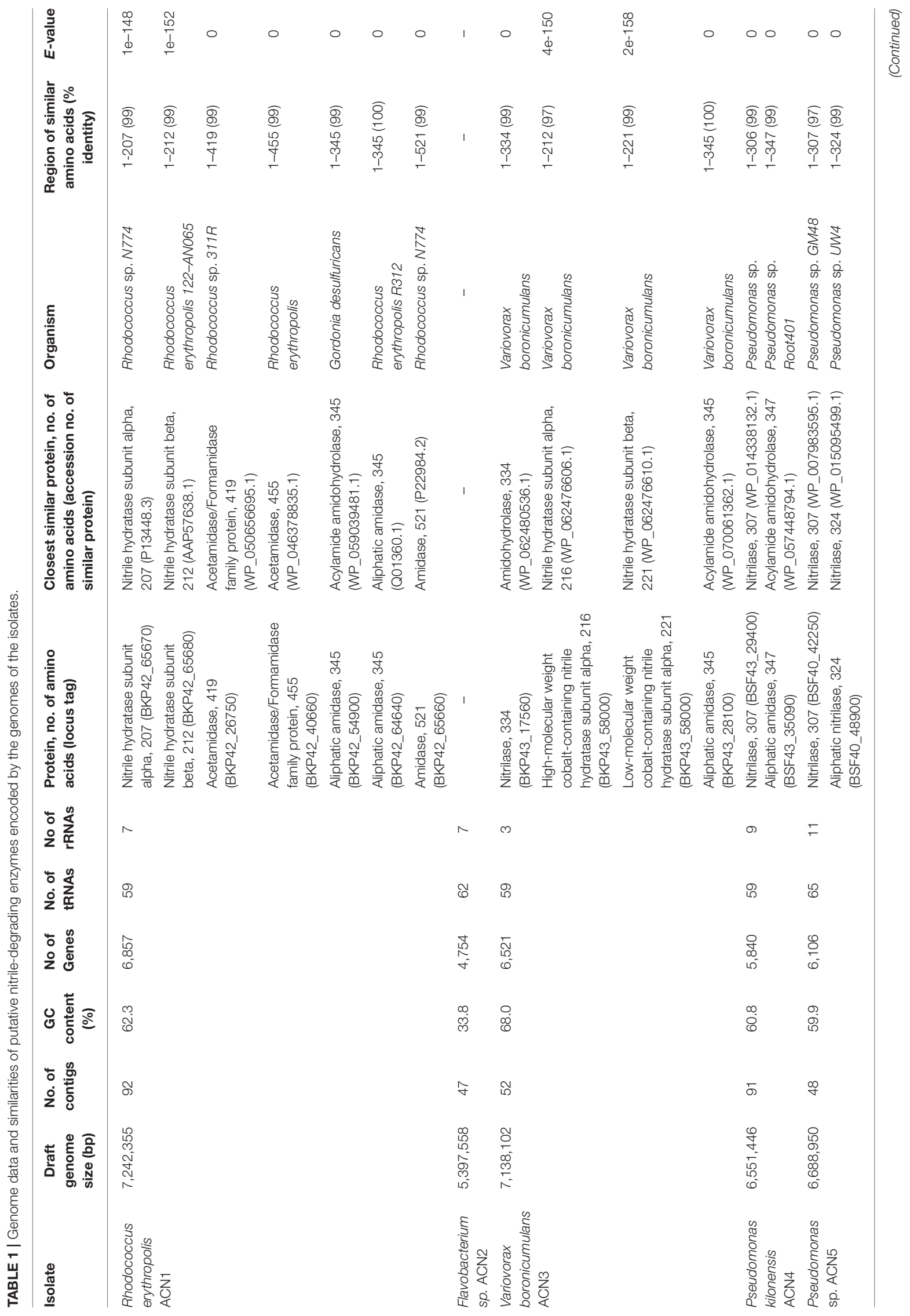




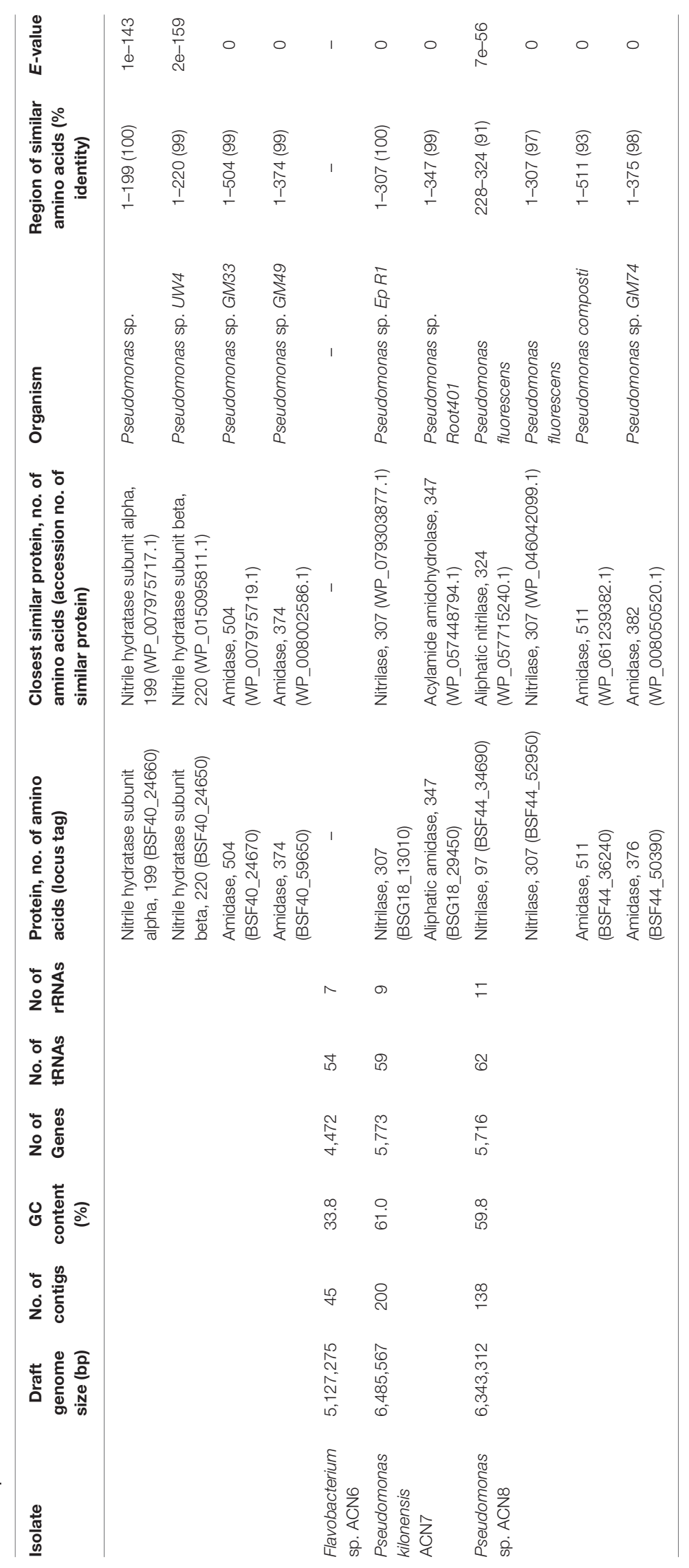


enrichment were Aeromonas (33\%) and Pseudomonas (55\%) with similar abundances in the control sample (Figure 1A). In the enrichment culture the relative abundances of Flavobacterium and Bacillus were 5 and 2\%, respectively, and in the control culture 2 and $0.4 \%$, respectively. Chryseobacterium, Paenibacillus, Pedobacter, and Sphingobacterium varied between 1.1 and $1.3 \%$ in the control culture, but showed relative abundances below $0.1 \%$ in the acetonitrile-containing enrichment. In contrast, Variovorax (3\%) was only detected in the acetonitrile enrichment culture in significant amounts. We performed isolation experiments from enrichment cultures on solid media with acetonitrile as sole nitrogen source. A total of 33 isolates (Figure 1B) were recovered of which eight isolates (ACN1 to ACN8, Table 1) showed a different $16 \mathrm{~S}$ rRNA gene sequence. All isolates were able to grow with acetonitrile as sole nitrogen source in axenic culture. No growth was observed in the absence of an added nitrogen source. The genomes of all eight isolates were sequenced and analyzed with the focus on genes potentially involved in nitrile-degradation (Table 1). Based on average nucleotide identity, tetra-nucleotide signatures and in silico DNA-DNA hybridization, the eight isolates were affiliated to the genera Flavobacterium, Pseudomonas, Rhodococcus and Variovorax. Besides Rhodococcus all isolated strains belonged to genera detected during 16S rRNA gene analysis of the bacterial community in the enrichment culture.

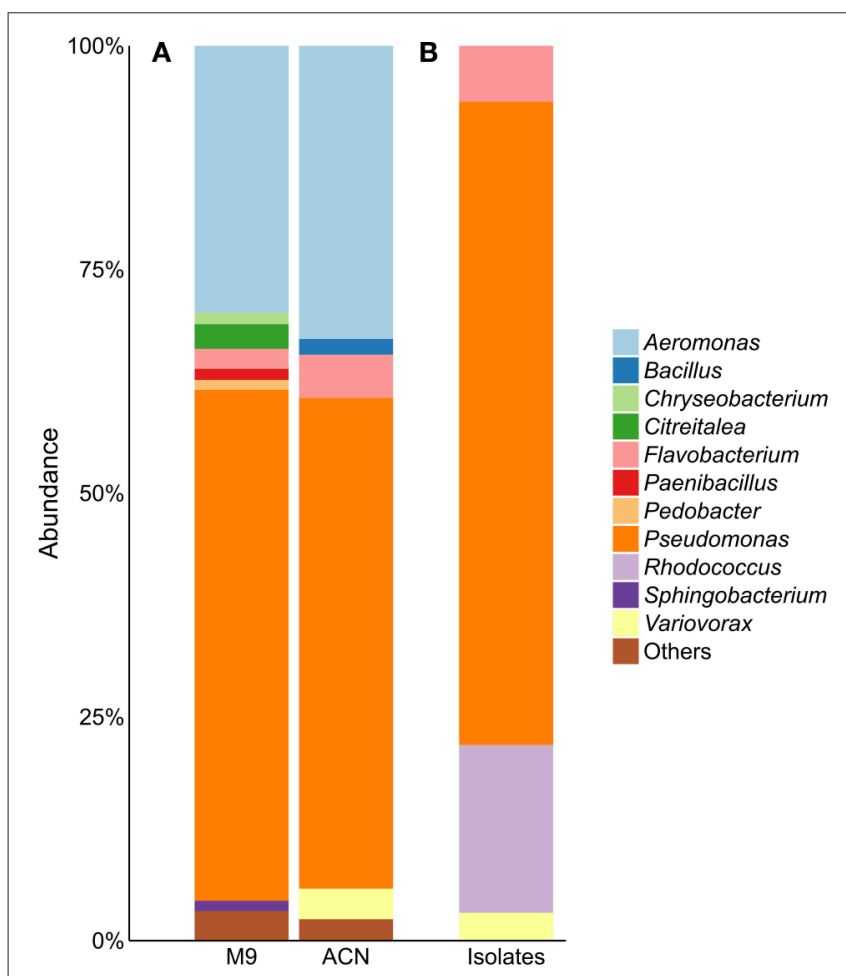

FIGURE 1 | (A) Community composition of acetonitrile-enrichment and control culture and (B) 16S rRNA gene analysis of 33 acetonitrile-degrading isolates; "Others" refers to genera with an abundance of $<1 \%$. M9, control; ACN, acetonitril enrichment.

\section{Rhodococcus (ACN1)}

In contrast to all other isolates, only slight traces of Rhodococcus could be found during 16S rRNA gene analysis. Nevertheless, this isolate was obtained multiple times from the enrichment, indicating its ability to degrade acetonitrile. The reason for the discrepancy between $16 \mathrm{~S}$ rRNA gene analysis of the community and the isolation results may be due to non-optimal media conditions for Rhodococcus in the enrichment culture, leading to low abundance. Genome sequencing of $R$. erythropolis ACN1 and quality-filtering resulted in 2,641,652 paired-end reads resulting in a draft genome of 7.24 Mbp with a 93.9fold coverage. Similarity searches for putative genes involved in nitrile-degradation revealed two genes (BKP42_65670 and BKP42_65680), coding for one of the two putative NHase subunits. These enzymes are known to be responsible for nitrile degradation in various $R$. erythropolis strains (Kaufmann et al., 1999; Brandão et al., 2003; Vejvoda et al., 2007; Kamble et al., 2013). The deduced protein sequences showed highest identity to the alpha subunit of Rhodococcus sp. N-774 (Ikehata et al., 1989) and the beta subunit of $R$. erythropolis deepsea strain 122-AN065 (Brandão et al., 2003). The deduced acetamidases (BKP42_40660 and BKP42_26750) are most similar to acetamidases of $R$. erythropolis and Rhodococcus sp. 311R (Ehsani et al., 2015). The two aliphatic amidases of the isolated strain (BKP42_54900 and BKP42_64640) most resemble an acylamide amidohydrolase from Gordonia desulfuricans and an amidase of Rhodococcus sp. R312 (Fournand et al., 1998). An additional amidase (BKP42_65660) is most similar to an amidase of Rhodococcus sp. N-774 (Table 1).

\section{Variovorax (ACN3)}

Members of the genus Variovorax were prime candidates for active nitrile degradation, as the genus appeared only in significant abundances in the bacterial community after enrichment with acetonitrile. With the isolation of ACN3 identified as $V$. boronicumulans a member of this genus was recovered. Sequencing and assembly of 3,471,160 paired-end reads resulted in a draft genome of $7.14 \mathrm{Mbp}$ (131-fold coverage). Although $V$. boronicumulans is a species described just recently (Miwa et al., 2008), evidence for nitrile degradation by this microorganism was reported before (Zhang et al., 2012; Liu et al., 2013). A search for genes encoding putative nitriledegrading enzymes resulted in the identification of genes for two possible degradation pathways, the first one via a nitrilase (BKP43_17560) and the second one via an NHase (BKP43_58000 and BKP43_58010) and amidase (BKP43_28100). The most similar enzymes to the nitrilase, alpha and beta subunits of NHase, and the aliphatic amidase are an amidohydrolase, NHase subunits and acylamide amidohydrolase of another $V$. boronicumulans strain, respectively (Table 1).

\section{Pseudomonas (ACN4, ACN5, ACN7, and ACN8)}

With a relative abundance of over 50\%, Pseudomonas is the major genus of the bacterial community in the acetonitrile enrichment culture. Several Pseudomonas species such as P. chlorographis and $P$. fluorescens are able to use nitriles as nitrogen source 
and harbor genes encoding nitrilases, or NHases and amidases (Nagasawa et al., 1987; Kiziak et al., 2005; Howden et al., 2009). The presence of these genes could be due to the plant habitat of some Pseudomonas species, which is rich in different and unusual nitriles like indole-3-carbonyl nitrile (Rajniak et al., 2015).

Isolates ACN4 and ACN7 were affiliated to P. kilonensis, a species described in 2001 (Sikorski et al., 2001). Sequencing of the ACN4 genome resulted in 2,239,346 paired-end reads, an average coverage of 96.7 -fold, and a draft genome of $6.55 \mathrm{Mbp}$. Genes encoding a putative nitrilase (BSF43_29400) and amidase (BSF43_35090) were detected, which showed highest identity to a Pseudomonas nitrilase and a putative acylamide amidohydrolase of $P$. sp. Root401 (Bai et al., 2015), respectively.

The genome assembly of isolate ACN7 was based on 2,049,404 paired-end reads and resulted in a draft genome of $6.49 \mathrm{Mbp}$ and a 74.9 -fold coverage. The GC-content is $61.0 \%$. The draft genome encodes a nitrilase (BSG18_13010) and an amidase (BSG18_29450) similar to a nitrilase of $P$. sp. Ep R1 (Chiellini et al., 2014) and an acylamide amidohydrolase of $P$. sp. Root401, respectively.

The two Pseudomonas isolates ACN5 and ACN8 could not be assigned to a specific Pseudomonas species. The genome of ACN5 (6.69 Mbp, 61.3-fold coverage) was assembled from 1,350,588 paired-end reads. Potential genes for two nitrilases, one NHase and two amidases were predicted in the ACN5 genome. The aliphatic nitrilase (BSF40_48900) showed highest similarity to a nitrilase of Pseudomonas sp. UW4 (Duan et al., 2013), while the other nitrilase (BSF40_42250) most resembles a nitrilase of Pseudomonas sp. GM48 (Brown et al., 2012). The NHase alpha and beta subunits (BSF40_24660 and BSF40_24650) are related to the corresponding ones of Pseudomonas and P. sp. UW4. The predicted amidases (BSF40_24670 and BSF40_59650) are similar to an amidase of $P$. sp. GM33 and GM49 (Brown et al., 2012). For the assembly of the ACN8 genome, $1,505,826$ pairedend reads were used, resulting in a draft genome of $6.34 \mathrm{Mbp}$ and 66.0-fold coverage. Genes for two nitrilases (BSF44_34690 and BSF44_52950) were predicted, which resemble two different nitrilases from $P$. fluorescens. Furthermore, two genes encoding putative amidases (BSF44_36240 and BSF44_50390) similar to an amidase of $P$. composti and P. sp. GM74 (Brown et al., 2012) were detected.

\section{Flavobacterium (ACN2 and ACN6)}

The two isolates ACN2 and ACN6 from the acetonitrile enrichment culture were affiliated to the Gram-negative genus Flavobacterium. A total of 1,503,824 paired-end reads were used for the assembly of the ACN2 genome, resulting in a draft genome of $5.40 \mathrm{Mbp}$ with an average coverage of 72.7fold. ACN6 genome assembly of 2,409,950 paired-end reads yielded a draft genome of $5.13 \mathrm{Mbp}$ with average coverage of 120.4-fold. Genes encoding putative nitrile-degrading enzymes were not detected in both draft genomes. As the nitrile degrading capacity of both strains was independently verified multiple times by growth experiments with acetonitrile as sole nitrogen source, the presence of so far unknown genes and pathways for nitrile utilization is indicated. In addition, the ability to overcome the nitrogen limitation via nitrogen fixation from air was experimentally excluded as no growth could be monitored in liquid M9 medium without any added nitrogen source. Furthermore, typical genes for nitrogen fixation such as dinitrogenases and dinitrogenase reductases were not identified in the genome sequences. Thus, two explanations are possible for the discrepancy between the observed phenotype of both strains and the lack of putative genes for nitrile degradation. First, genes responsible for nitrile degradation could not be annotated as they are located in contig gaps, as both genomes are still in the draft state. This is unlikely as most contig gaps are due to repetitive regions longer than the read length of the used sequencing technology (Whiteford et al., 2005; Chaisson et al., 2015). Second, genes for new types of nitrile degradation enzymes are present. The only other available study on nitrile degradation by Flavobacteria reports weak degradation of 3-cyanopyridine by F. aquatile IFO 3772, F. suaveolens IFO 3752 and F. rigense IAM 1238 (Kato et al., 2000), but genes responsible for the observed phenotype were not reported and genomes of the three strains are not available. Thus, further analyses are required to unravel the basis of the nitrile-degrading phenotype of the flavobacterial isolates.

\section{CONCLUSION}

The here presented data revealed the potential of specifically designed enrichment experiments for the isolation of organisms with a desired metabolic activity like nitrile degradation. Our study provided new candidates to cover the industrial demand for new nitrile-degrading biocatalysts as part of a green chemistry. While for the isolates $R$. erythropolis ACN1, V. boronicumulans ACN2, P. kilonensis ACN4 and ACN7 as well as P. sp. ACN5 and ACN8 nitrilases and/or NHases and amidases could be annotated, the nitrile-degrading pathway of $F$. sp ACN2 and ACN6 remains to be unraveled.

\section{ACCESSION NUMBERS}

The draft genome sequences of all eight organisms have been deposited at GenBank with the following accession numbers: R. erythropolis ACN1, MRCL00000000; F. sp. ACN2, MRCM00000000; V. boronicumulans ACN3, MRCN00000000; P. kilonensis ACN4, MRCO00000000; $P$. sp. ACN5, MRCP00000000; F. sp. ACN6, MRCQ00000000; P. kilonensis ACN7, MRCR00000000; P. sp. ACN8, MRCS00000000. Raw sequence data of all genomes are available at the NCBI SRA archive and linked to the respective BioSamples. The sequences of the 16S rRNA gene analysis are linked to the BioSamples SAMN07278921 (inoculum), SAMN0278947 (control) and SAMN07278950 (acetonitrile enrichment). Strains have been submitted to the DSMZ German Collection of Microorganism and Cell Cultures and are available on request.

\section{AUTHOR CONTRIBUTIONS}

$\mathrm{RD}$ and RH conceived the study. RE performed the experiments. $\mathrm{RE}$ and DS analyzed the data. All authors interpreted the results, 
wrote the manuscript and reviewed the final version of the manuscript.

\section{FUNDING}

The work of RE was supported by the "Fonds der Chemischen Industrie im Verband der Chemischen Industrie e.V." The funders had no role in study design, data collection and interpretation, or the decision to submit the work for publication.

\section{REFERENCES}

Altschul,., S. F., Gish, W., Miller, W., Myers, E. W., and Lipman, D. J. (1990). Basic local alignment search tool. J. Mol. Biol. 215, 403-410. doi: 10.1016/S002 2-2836(05)80360-2

Andrews, S. (2010). FastQC: A Quality Control Tool for High throughput Sequence Data. Available online at: http://www.bioinformatics.babraham.ac.uk/projects/ fastqc/

Atlas, R. M. (2010). Handbook of Microbiological Media, 4th Edn. Boca Raton, FL: CRC Press.

Bai, Y., Müller, D. B., Srinivas, G., Garrido-Oter, R., and Potthoff, E., Rott., M., et al. (2015). Functional overlap of the Arabidopsis leaf and root microbiota. Nature 528, 364-369. doi: 10.1038/nature16192

Bankevich, A., Nurk, S., Antipov, D., Gurevich, A. A., Dvorkin, M., Kulikov, A. S., et al. (2012). SPAdes: a new genome assembly algorithm and its applications to single-cell sequencing. J. Comput. Biol. 19, 455-477. doi: $10.1089 / \mathrm{cmb} .2012 .0021$

Bolger, A. M., Lohse, M., and Usadel, B. (2014). Trimmomatic: a flexible trimmer for Illumina sequence data. Bioinformatics 30, 2114-2120. doi: 10.1093/bioinformatics/btu170

Brandão, P. F., Clapp, J. P., and Bull, A. T. (2003). Diversity of nitrile hydratase and amidase enzyme genes in Rhodococcus erythropolis recovered from geographically distinct habitats. Appl. Environ. Microbiol. 69, 5754-5766. doi: 10.1128/AEM.69.10.5754-5766.2003

Brown, S. D., Utturkar, S. M., Klingeman, D. M., Johnson, C. M., Martin, S. L., Land, M. L., et al. (2012). Twenty-one genome sequences from Pseudomonas species and 19 genome sequences from diverse bacteria isolated from the rhizosphere and endosphere of Populus deltoids. J. Bacteriol. 194, 5991-5993. doi: 10.1128/JB.01243-12

Caporaso, J. G., Kuczynski, J., Stombaugh, J., Bittinger, K., Bushman, F. D., Costello, E. K., et al. (2010). QIIME allows analysis of highthroughput community sequencing data. Nat. Methods 7, 335-336. doi: $10.1038 /$ nmeth.f.303

Chaisson, M. J. P., Wilson, R. K., and Eichler, E. E. (2015). Genetic variation and the de novo assembly of human genomes. Nat. Rev. Genet. 16, 627-640. doi: $10.1038 / \mathrm{nrg} 3933$

Chiellini, C., Maida, I., Emiliani, G., Mengoni, A., Mocali, S., Fabiani, A., et al. (2014). Endophytic and rhizospheric bacterial communities isolated from the medicinal plants Echinacea purpurea and Echinacea angustifolia. Int. Microbiol. 17, 165-174. doi: 10.2436/20.1501.01.219

Clouthier, C. M., and Pelletier, J. N. (2012). Expanding the organic toolbox: a guide to integrating biocatalysis in synthesis. Chem. Soc. Rev. 41, 1585-1605. doi: $10.1039 / \mathrm{c} 2 \mathrm{cs} 15286 \mathrm{j}$

Duan, J., Jiang, W., Cheng, Z., Heikkila, J. J., and Glick, B. R. (2013). The complete genome sequence of the plant growth-promoting bacterium Pseudomonas sp. UW4. PLoS ONE 8:e58640. doi: 10.1371/journal.pone.0058640

Edgar, R. C. (2010). Search and clustering orders of magnitude faster than BLAST. Bioinformatics 26, 2460-2461. doi: 10.1093/bioinformatics/btq461

Ehsani, E., Jauregui, R., Geffers, R., Jareck, M., Boon, N., Pieper, D. H., et al. (2015). Draft genome sequence of Rhodococcus sp. strain 311R. Genome Announc. 3:e00378-e00415. doi: 10.1128/genomeA.00378-15

\section{ACKNOWLEDGMENTS}

We thank Melanie Heinemann for technical support. We acknowledge support by the Open Access Publication Funds of the University of Goettingen.

\section{SUPPLEMENTARY MATERIAL}

The Supplementary Material for this article can be found online at: http://journal.frontiersin.org/article/10.3389/fenvs. 2017.00056/full\#supplementary-material

Fleming, F. F. (1999). Nitrile-containing natural products. Nat. Prod. Rep. 16, 567-606. doi: 10.1039/a804370a

Fournand, D., Araud, A., and Galzy, P. (1998). Study of the acyl transfer activity of a recombinant amidase overproduced in an Escherichia coli strain. Application for short-chain hydroxamic acid and acid hydrazide synthesis. J. Mol. Catal. B Enzym. 4, 77-90. doi: 10.1016/S1381-1177(97) 00024-6

Gong, J. S., Lu, Z. M., Li, H., Shi, J. S., Zhou, Z. M., and Xu, Z. H. (2012). Nitrilases in nitrile biocatalysis: recent progress and forthcoming research. Microb. Cell Fact. 11:142. doi: 10.1186/1475-2859-11-142

Howden, A. J., Harrison, C. J., and Preston, G. M. (2009). A conserved mechanism for nitrile metabolism in bacteria and plants. Plant J. 57, 243-253. doi: 10.1111/j.1365-313X.2008.03682.x

Ikehata, O., Nishiyama, M., Horinouchi, S., and Beppu, T. (1989). Primary structure of nitrile hydratase deduced from the nucleotide sequence of a Rhodococcus species and its expression in Escherichia coli. Eur. J. Biochem. 181, 563-570. doi: 10.1111/j.1432-1033.1989.tb14761.x

Kamble, A. L., Banoth, L., Meena, V. S., Singh, A., Chisti, Y., and Banerjee, U. C. (2013). Nitrile hydratase of Rhodococcus erythropolis: characterization of the enzyme and the use of whole cells for biotransformation of nitriles. 3 Biotech. 3, 319-330. doi: 10.1007/s13205-012-0104-2

Kato, Y., Ooi, R., and Asano, Y. (2000). Distribution of aldoxime dehydratase in microorganisms. Appl. Environ. Microbiol. 66, 2290-2296. doi: 10.1128/AEM.66.6.2290-2296.2000

Kaufmann, G., Dautzenberg, H., Henkel, H., Müller, G., Schäfer, T., Undeutsch, et al. (1999). Nitrile hydratase from Rhodococcus erythropolis: metabolization of steroidal compounds with a nitrile group. Steroids 64, 535-540. doi: 10.1016/S0039-128X(99)00028-8

Kiziak, C., Conradt, D., Stolz, A., Mattes, R., and Klein, J. (2005). Nitrilase from Pseudomonas fluorescens EBC191: cloning and heterologous expression of the gene and biochemical characterization of the recombinant protein. Microbiology 151, 3639-3648. doi: 10.1099/mic.0.28246-0

Klindworth, A., Pruesse, E., Schweer, T., Peplies, J., Quast, C., Horn, M., et al. (2013). Evaluation of general 16S ribosomal RNA gene PCR primers for classical and next-generation sequencing-based diversity studies. Nucleic Acids Res. 41:e1. doi: 10.1093/nar/gks808

Langmead, B., and Salzberg, S. L. (2012). Fast gapped-read alignment with Bowtie 2. Nat. Methods 9, 357-359. doi: 10.1038/nmeth.1923

Li, C., Yue, Z., Feng, F., Xi, C., Zang, H., An, X., et al. (2016). A novel strategy for acetonitrile wastewater treatment by using a recombinant bacterium with biofilm-forming and nitrile-degrading capability. Chemosphere 161, 224-232. doi: 10.1016/j.chemosphere.2016.07.019

Liu, Z.-H., Cao, Y.-M., Zhou, Q.-W., Gui, K., Ge, F., Hou, J.-Y., et al. (2013). Acrylamide biodegradation ability and plant growth-promoting properties of Variovorax boronicumulans CGMCC 4969. Biodegradation 24, 855-864. doi: 10.1007/s10532-013-9633-6

Martin, M. (2011). Cutadapt removes adapter sequences from high-throughput sequencing reads. Embnet. J. 17, 10-12. doi: 10.14806/ej.17.1.200

Meier-Kolthoff, J. P., Auch, A. F., Klenk, H. P., and Göker, M. (2013). Genome sequence-based species delimination with confidence intervals and improved distance functions. BMC Bioinformatics 14:60. doi: 10.1186/1471-2105-14-60 
Miwa, H., Ahmed, I., Yoon, J., Yokota, A., and Fujiwara, T. (2008). Variovorax boronicumulans sp. nov., a boron-accumulating bacterium isolated from soil. Int. J. Syst. Evol. Microbiol. 58, 286-289. doi: 10.1099/ijs.0.65315-0

Nagasawa, T., Nanba, H., Ryuno, K., Takeuchi, K., and Yamada, H. (1987). Nitrile hydratase of Pseudomonas chlororaphis B23. Purification and characterization. Eur. J. Biochem. 162, 691-698. doi: 10.1111/j.1432-1033.1987.tb10692.x

Panova, A., Mersinger, L. J., Liu, Q., Foo, T., Roe, D. C., Spillan, W. L., et al. (2007). Chemoenzymatic synthesis of glycolic acid. Adv. Synth. Catal. 349, 1462-1474. doi: 10.1002/adsc.200700061

Rajniak, J., Barco, B., Clay, N. K., and Sattely, E. S. (2015). A new cyanogenic metabolite in Arabidopsis required for inducible pathogen defence. Nature 525, 376-379. doi: 10.1038/nature14907

Richter, M., Rosselló-Móra, R., Glöckner, F. O., and Peplies, J. (2016). JSpecies WS: a web server for prokaryotic species circumscription based on pairwise genome comparison. Bioinformatics 32, 929-931. doi: 10.1093/bioinformatics/ btv681

Schmid, A., Dordick, J. S., Hauer, B., Kiener, A., Wubbolts, M., and Witholt, B. (2001). Industrial biocatalysis today and tomorrow. Nature 409, 258-268. doi: $10.1038 / 35051736$

Seemann, T. (2014). Prokka: rapid prokaryotic genome annotation. Bioinformatics 30, 2068-2069. doi: 10.1093/bioinformatics/btu153

Sikorski, J., Stackebrandt, E., and Wackernagel, W. (2001). Pseudomonas kilonensis sp. nov., a bacterium isolated from agricultural soil. Int. J. Syst. Evol. Microbiol. 51, 1549-1555. doi: 10.1099/00207713-514-1549

Vejvoda, V., Šveda, O., Kaplan, O., Přikrylová, V., Elišáková, V., Himl, M., et al. (2007). Biotransformation of heterocyclic dinitriles by Rhodococcus erythropolis and fungal nitrilases. Biotechnol. Lett. 29, 1119-1124. doi: 10.1007/s10529-007-9364-Z
Vergne-Vaxelaire, C., Bordier, F., Fossey, A., Besnard-Gonnet, M., Debard, A., Mariage, A., et al. (2013). Nitrilase activity screening on structurally diverse substrates: providing biocatalytic tools for organic synthesis. Adv. Synth. Catal. 355, 1763-1779. doi: 10.1002/adsc.201201098

Whiteford, N., Haslam, N., Weber, G., Prügel-Bennett, A., Essex, J. W., Roach, P. L., et al. (2005). An analysis of the feasibility of short read sequencing. Nucleic Acids Res. 33:e171. doi: 10.1093/nar/gnil70

Yilmaz, P., Parfrey, L. W., Yarza, P., Gerken, J., Pruesse, E., Quast, C., et al. (2014). The SILVA and "All-species Living Tree Project (LTP)" taxonomic frameworks. Nucleic Acids Res. 42, D643-D648. doi: 10.1093/nar/gkt1209

Zhang, H.-J., Zhou, Q.-W., Zhou, G.-C., Cao, Y.-M., Dai, Y.-J., Ji, W.-W., et al. (2012). Biotransformation of the neonicotinoid insecticide Thiacloprid by the bacterium Variovorax boronicumulans strain J1 and mediation of the major metabolic pathway by nitrile hydratase. J. Agric. Food Chem. 60, 153-159. doi: $10.1021 /$ jf203232u

Zhang, J., Kobert, K., Flouri, T., and Stamatakis, A. (2014). PEAR: a fast and accurate Illumina Paired-End reAd mergeR. Bioinformatics 30, 614-620. doi: 10.1093/bioinformatics/btt593

Conflict of Interest Statement: The authors declare that the research was conducted in the absence of any commercial or financial relationships that could be construed as a potential conflict of interest.

Copyright (c) 2017 Egelkamp, Schneider, Hertel and Daniel. This is an open-access article distributed under the terms of the Creative Commons Attribution License (CC $B Y)$. The use, distribution or reproduction in other forums is permitted, provided the original author(s) or licensor are credited and that the original publication in this journal is cited, in accordance with accepted academic practice. No use, distribution or reproduction is permitted which does not comply with these terms. 


\subsection{From sequence to function:}

\section{A new workflow for nitrilase identification}

Richard Egelkamp ${ }^{1 *}$, Ines Friedrich ${ }^{1 *}$, Robert Hertel $^{1}$ \& Rolf Daniel ${ }^{1}$

${ }^{1}$ Genomic and Applied Microbiology \& Göttingen Genomics Laboratory, Institute of Microbiology and Genetics, University of Göttingen, Göttingen, Germany ${ }^{*} \mathrm{RE}$ and IF contributed equally to this work

\section{Supplementary data can be found on the enclosed DVD}

\section{Author contributions}

Performed the experiments: IF

Analyzed the data: RE, IF

Wrote the publication: RE, IF, RH, RD

Conceived the study: RE, RH, RD 


\section{ABSTRACT}

This study describes a workflow for simple and fast acquisition of nitrilase candidates from large metagenomic sequence sets. For identification of active enzymes, a NADH-coupled high-throughput assay was established. Purification of enzymes could be omitted as crude extract containing the overexpressed putative nitrilases was used. In addition, long incubation times could be avoided by combination of nitrile and NADH conversion in a single reaction. This allowed the direct measurement of nitrile degradation and provides not only insights into substrate specificity but also in degradation efficiency. The novel assay was subsequently used for investigation of seventy putative nitrilases previously identified during sequence-based screens of metagenomes derived from nitrile-treated microbial communities. Six enzymes showed activity and the most interesting nitrilase was purified and analyzed. The activity of the characterized arylacetonitrilase exhibited a broad $\mathrm{pH}$ range and a high long-term stability. The enzyme showed high specificity for phenylacetonitrile with $K_{\mathrm{M}}$ of $1.29 \mathrm{mM}$ and $V_{\max }$ of $13.85 \mathrm{U} / \mathrm{mg}$ protein. In conclusion, we provide a setup for simple and rapid analysis of putative nitrilases from sequence to function, demonstrated by the identification, isolation, and characterization of the here described arylacetonitrilase.

\section{INTRODUCTION}

Nitriles are often toxic compounds with $-\mathrm{C} \equiv \mathrm{N}$ as functional group. They are widespread in nature and present in plants in form of cyanoglycosides (1). Other naturally occurring nitriles are e.g. cyanohydrins in fungi and arthropods, different antibiotics in bacteria, or ricine and phenylacetonitrile in plants (2). For the enzymatic degradation of nitriles, two pathways are known. The first is the direct conversion of nitriles to corresponding carboxylic acids via nitrilases (EC 3.5.5.1) and the second the degradation of nitriles to corresponding amides via nitrile hydratases (NHases) (EC 4.2.1.84) and the subsequent hydrolysis of amides to carboxylic acids and ammonia using amidases (EC 3.5.1.4). Nitrile degrading catalysts can be found in many organisms including bacteria, filamentous fungi, yeasts, and plants (3-5). In industry, these enzymes are used for production of bulk chemicals such as nicotinic acid or glycolic acid $(6,7)$. Identification of novel nitrile-degrading enzymes is important due to their industrial use and the often observed substrate and product inhibition under production conditions (8-11). Many nitrile-degrading enzymes were found via (meta)genome mining techniques. A functional screening approach could be applied to identify nitrilase genes from plasmid libraries containing metagenomic DNA from different sources (12-14). In silico screening of (meta)genomic sequence data is used to identify putative nitrilases and nitrile hydratases in publicly available sequence space (15). Although sequence-based approaches lead very quickly to many new enzyme candidates, they remain to be predictions until functional verification. To overcome this limitation and take advantage of the 
constantly growing metagenomic sequence pool, procedures are needed for efficient verification of nitrilase activity.

The focus of this investigation was to establish a workflow which enables fast functional verification of nitrilases identified by sequence-based screenings. As starting material, six metagenomes which were obtained during determination of nitrile-influence on microbial communities (Chapter 3.1) were chosen. A procedure was established to reduce the 70 putative nitrilase-encoding genes derived from sequence-driven mining of the metagenomes to candidates with verified enzyme activity. Serving as prove of concept, one enzyme candidate was heterologously expressed and purified. Subsequently, the recovered enzyme and its activity were characterized including the determination of optimal $\mathrm{pH}$, temperature range, relative stability, and substrate specificity. In addition, influence of divalent ions and other substances on enzyme activity, and enzyme kinetics were determined.

\section{RESULTS}

Screening optimization. Most modern high-throughput nitrilase assays have drawbacks such as low sensitivity or work only under specific conditions. One of the most promising approaches is a NADHcoupled assay in which ammonia released by the nitrilase reaction is consumed by a glutamate dehydrogenase $(\mathrm{GDH})$ under conversion of $\mathrm{NADH}$ to $\mathrm{NAD}^{+}$. The conversion of this cofactor is monitored at $340 \mathrm{~nm}$ and reveals activity of the tested (putative) nitrilases (15). However, it requires extended incubation times for nitrilase reaction and provides only qualitative information and no insights regarding enzymatic efficiency. We modified the assay to allow fast and simple screening without additional incubation steps in a single reaction mixture. Therefore, concentration of all test ingredients was optimized to allow continuous measurement of NADH conversion in a 96-well plate, leading to first insights into efficiency of nitrile degradation. In comparison to the previous method, the amount of NADH was increased to $0.5 \mathrm{mM}$ for a larger measuring range and the concentration of $\alpha$-ketoglutarate was reduced to prevent inhibition of the GDH reaction. These modifications were tested by the use of an already characterized nitrilase from Rhodococcus rhodochrous $\mathrm{K} 22(16,17)$ (Figure 1).

To reduce identification of false positives or enzymes with low activity, a threshold was defined. During the first 15 minutes of measurement, total conversion of succinonitrile, fumaronitrile, and crotononitrile by the $\mathrm{K} 22$ nitrilase could be observed. As activity for these substrates was described before $(16,17)$, only reactions taking place in the first 15 minutes were considered as nitrilase activity during subsequent experiments. 


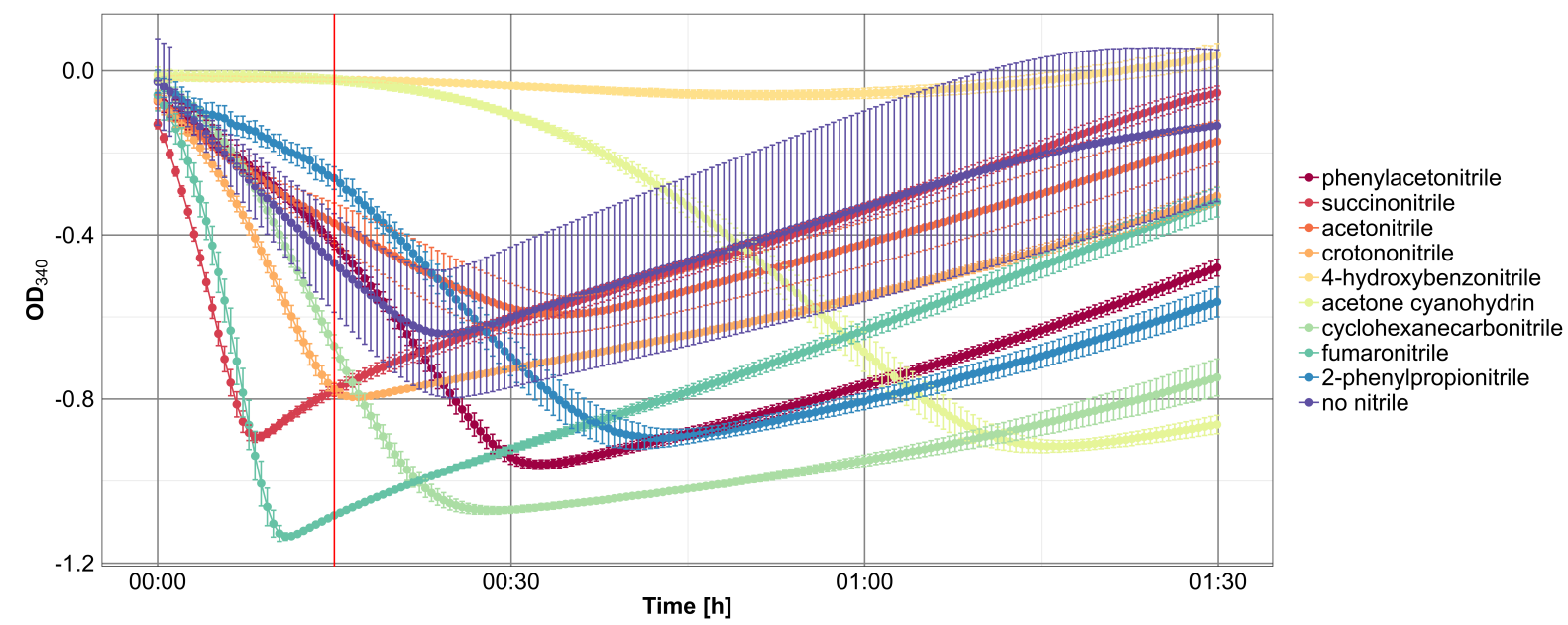

Figure 1: Nitrile degradation of the $\boldsymbol{R}$. rhodochrous $\mathrm{K} 22$ nitrilase. The assay was performed in triplicate at $37^{\circ} \mathrm{C}$ in a 96-well plate. NADH conversion was monitored at $340 \mathrm{~nm}$. Fumaronitrile, succinonitrile, and crotononitrile were degraded after $15 \mathrm{~min}$, thereby defining the threshold (red line) for the experimental time.

Analysis of putative nitrilases. Six metagenomes of microbial communities obtained from phenylacetonitrile-, succinonitrile-, acetonitrile-, crotononitrile-, 4-hydroxybenzonitrile-, or cyclohexanecarbonitrile-containing cultures (Chapter 3.1) served as source for putative nitrilases. In total, 70 annotated putative nitrilases were identified in these metagenomes and subsequently analyzed (Data Set S1). Initially, all enzyme candidates were compared with 46 reference nitrilases obtained from SWISS-Prot to relate the deduced protein sequences to already characterized enzymes. Results led to 13 protein clusters (Data Set S2) and reduced our data set to 60 unique candidates. The first three clusters contained characterized as well as putative nitrilases ranging from 5.96 to $38.68 \mathrm{kDa}, 12.7$ to $40.2 \mathrm{kDa}$, and 10.41 to $38.62 \mathrm{kDa}$. Six clusters consisted just of known and characterized nitrilases, whereas the remaining four clusters included just putative enzyme sequences. As the smallest characterized prokaryotic nitrilase (from Pyrococcus abyssi GE5) consists of 262 amino acids (18), all putative nitrilases smaller than this were checked for ribosomal binding sites (RBS) to exclude artificial peptides caused by incomplete metagenomic assembly. Finally, 37 candidates were selected for cloning and overexpression in E. coli.

After amplification of the respective genes from metagenomic DNA and sequence verification, frameshift mutations were observed in some cases and led to the exclusion of these candidates. In other cases, point mutations were observed, which were included into subsequent analyses as not every point mutation leads to non-functional proteins. Finally, a total number of 26 putative nitrilases were sub-cloned (Data Set S1). Subsequent overexpression and verification via SDS page revealed 13 putative nitrilases. 
The previously established assay was used to verify nitrilase activity with the 13 putative nitrilase candidates using nine different nitriles as substrate (Table 1). Six of the putative enzymes showed nitrilase activity for at least one nitrile. Most were active with succinonitrile or fumaronitrile. In addition, one converted the aromatic phenylacetonitrile (Figure 2). Thus, the workflow presented here has shown a way to successfully narrow down bioinformatically identified enzyme candidates to the interesting functional representatives.

Table 1: Overexpressed putative nitrilases. Metagenome: metagenomic origin of the genes. Variant: Variants obtained by point mutations (for sequences, see Data Set S1). Origin: gene sequences were used for a blastn search against the NCBI nr database and best hits are shown; Q: query cover; E: e value; I: ident; A: accession. Substrate: substrate determined with high-throughput assay. SUN: succinonitrile; CAN: acetonitrile; CRN: crotononitrile; CCN: cyclohexanecarbonitrile; PAN: phenylacetonitrile; FUN: fumaronitrile.

\begin{tabular}{|c|c|c|c|c|c|}
\hline $\begin{array}{l}\text { Meta- } \\
\text { genome }\end{array}$ & Nitrilase & Variant & Mass [kDa] & Origin & Substrate \\
\hline SUN & $\begin{array}{l}\text { D3C71- } \\
601570\end{array}$ & $B$ & 36.12 & $\begin{array}{l}\text { Variovorax boronicumulans } \\
\text { (Q: 100\%, E: 0.0, I: } 98 \%, \mathrm{~A}: \mathrm{KY} 937903 \text { ) }\end{array}$ & PAN \\
\hline SUN & $\begin{array}{l}\text { D3C71_ } \\
775930\end{array}$ & B & 33.0 & $\begin{array}{l}\text { Pseudomonas fluorescens } \\
\text { (Q: 99\%, E: 0.0, I: 87\%, A: CP010945) }\end{array}$ & - \\
\hline SUN & $\begin{array}{l}\text { D3C71_- } \\
946180\end{array}$ & $C$ & 35.13 & $\begin{array}{l}\text { Pseudomonas sp. } \\
\text { (Q: } 99 \%, \mathrm{E}: 0.0, \mathrm{I}: 94 \%, \mathrm{~A}: \mathrm{CP} 003880 \text { ) }\end{array}$ & SUN, FUN \\
\hline $\mathrm{ACN}$ & $\begin{array}{l}\mathrm{D} 3 \mathrm{C} 72_{-} \\
113100\end{array}$ & $A$ & 38.7 & $\begin{array}{l}\text { Janthinobacterium sp. } \\
\text { (Q: } 99 \%, \mathrm{E}: 0.0, \mathrm{I}: 87 \%, \mathrm{~A}: \mathrm{CP} 000269 \text { ) }\end{array}$ & - \\
\hline $\mathrm{ACN}$ & $\begin{array}{l}\mathrm{D} 3 \mathrm{C} 72_{-} \\
306600\end{array}$ & $A$ & 38.62 & $\begin{array}{l}\text { Pseudomonas sp. } \\
\text { (Q: 97\%, E: 0.0, I: 89\%, A: AP017423) }\end{array}$ & SUN, FUN \\
\hline $\mathrm{ACN}$ & $\begin{array}{l}\mathrm{D} 3 \mathrm{C} 72_{-} \\
447890^{-}\end{array}$ & $A$ & 32.93 & $\begin{array}{l}\text { Pseudomonas fluorescens } \\
\text { (Q: 99\%, E: 0.0, I: 87\%, A: CP010945) }\end{array}$ & - \\
\hline $\mathrm{ACN}$ & $\begin{array}{l}\text { D3C72 } \\
810700\end{array}$ & $C$ & 36.74 & $\begin{array}{l}\text { Cupriavidus basilensis } \\
\text { (Q: } 98 \%, \mathrm{E}: 0.0, \mathrm{I}: 87 \%, \mathrm{~A}: \mathrm{CP} 010537 \text { ) }\end{array}$ & SUN, FUN \\
\hline CRN & $\begin{array}{ll}\mathrm{D} 3 \mathrm{C} 73_{-} \\
384910\end{array}$ & $A$ & 35.2 & $\begin{array}{l}\text { Pseudomonas sp. } \\
\text { (Q: } 100 \%, \mathrm{E}: 0.0, \mathrm{I}: 98 \%, \mathrm{~A}: \mathrm{CP} 003880 \text { ) }\end{array}$ & SUN, FUN \\
\hline CRN & $\begin{array}{ll}\mathrm{D} 3 \mathrm{C} 73_{-} \\
384910\end{array}$ & $B$ & 35.18 & $\begin{array}{l}\text { Pseudomonas sp. } \\
\text { (Q: } 100 \%, \mathrm{E}: 0.0, \mathrm{l}: 98 \%, \mathrm{~A}: \mathrm{CP} 003880 \text { ) }\end{array}$ & SUN, FUN \\
\hline CRN & $\begin{array}{l}\mathrm{D} 3 \mathrm{C} 73 \\
406050\end{array}$ & $A$ & 32.84 & $\begin{array}{l}\text { Pseudomonas sp. } \\
\text { (Q: } 100 \%, \mathrm{E}: 0.0, \mathrm{I}: 95 \%, \mathrm{~A}: \mathrm{CP} 003880 \text { ) }\end{array}$ & - \\
\hline CRN & $\begin{array}{l}\text { D3C73 } \\
406050\end{array}$ & $B$ & 32.86 & $\begin{array}{l}\text { Pseudomonas sp. } \\
\text { (Q: } 100 \%, \mathrm{E}: 0.0, \mathrm{I}: 91 \%, \mathrm{~A}: \mathrm{CP} 003880 \text { ) }\end{array}$ & FUN \\
\hline CRN & $\begin{array}{l}\text { D3C73 } \\
579180\end{array}$ & $\mathrm{G}$ & 32.91 & $\begin{array}{l}\text { Pseudomonas sp. } \\
\text { (Q: } 100 \%, \mathrm{E}: 0.0, \mathrm{I}: 97 \%, \mathrm{~A}: \mathrm{CP} 003880 \text { ) }\end{array}$ & - \\
\hline $\mathrm{CCN}$ & $\begin{array}{l}\text { D3C75_ } \\
459950\end{array}$ & $\mathrm{~B}$ & 32.83 & $\begin{array}{l}\text { Pseudomonas sp. } \\
\text { (Q: } 100 \%, \mathrm{E}: 0.0, \mathrm{I}: 91 \%, \mathrm{~A}: \mathrm{CP} 003880 \text { ) }\end{array}$ & - \\
\hline
\end{tabular}




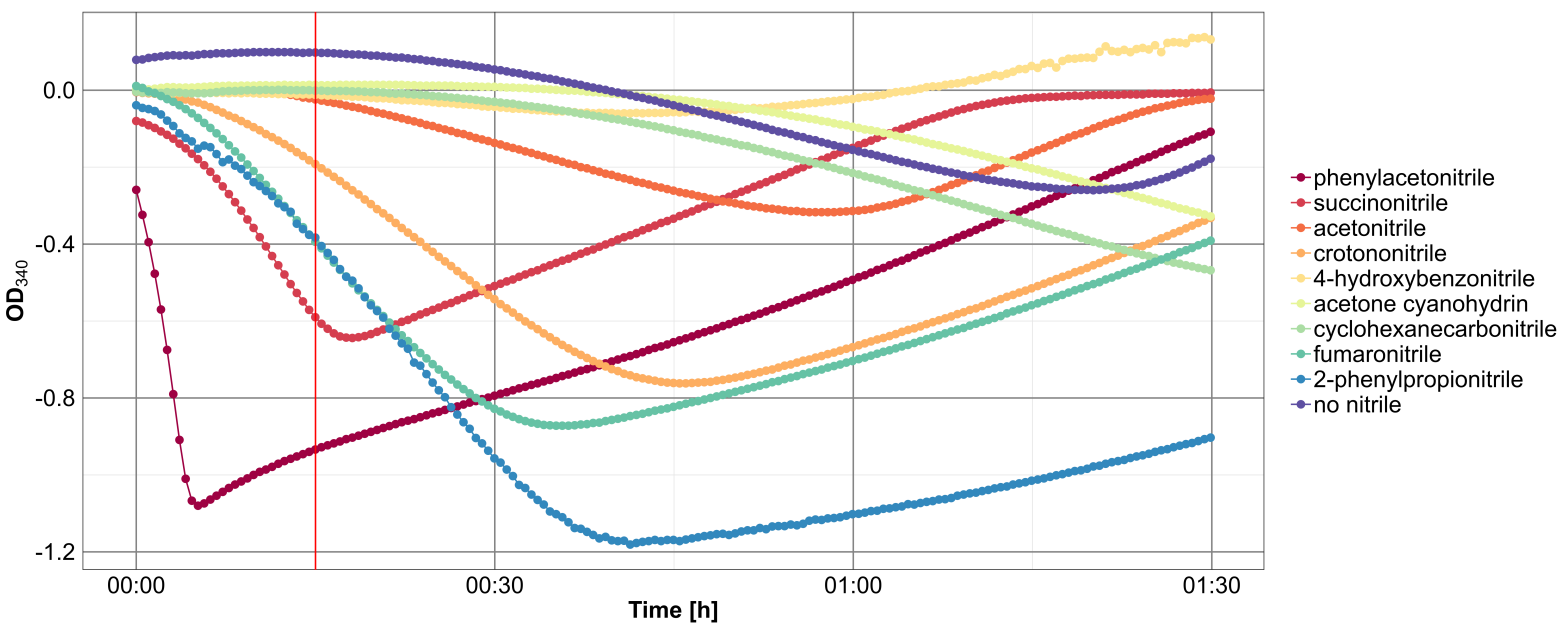

Figure 2: Substrate specificity of enzyme D3C71_601570. The assay was performed at $37^{\circ} \mathrm{C}$ in a 96well plate. NADH conversion was monitored at $340 \mathrm{~nm}$. Degradation of phenylacetonitrile was observed until the 15 min threshold (red line).

Characterization of enzyme D3C71_601570. To prove the previous observations, we chose the phenylacetonitrile-degrading enzyme candidate for detailed characterization and verification of enzymatic activity under defined conditions. For further experimental characterization, a sequence encoding a $\mathrm{His}_{6}$ tag was added to the $\mathrm{N}$-terminal end of the gene. Subsequently, the enzyme was produced and purified. SDS-PAGE analysis of the enzyme preparation confirmed the from the sequence deduced molecular mass of $36 \mathrm{kDa}$. The purified enzyme was subjected to a detailed characterization with focus on optimal enzymatic conditions, stability, and kinetics. Phenylacetonitrile was used as standard substrate, as the nitrilase showed only activity for this compound during highthroughput screening.

Investigations regarding $\mathrm{pH}$ dependency showed $\mathrm{pH} 6$ to be optimal, whereby at $\mathrm{pH} 5.5$ to 8 about $90 \%$ enzyme activity could still be observed. The limits of the enzyme were observed at pH 5 and 10.5, as only $20 \%$ activity remained (Figure $3 \mathrm{~A}$ ). Optimum temperature of the enzyme was determined in the range of $25^{\circ} \mathrm{C}$ to $60{ }^{\circ} \mathrm{C}$ at $\mathrm{pH}$. Highest activity was measured between $45^{\circ} \mathrm{C}$ and $55^{\circ} \mathrm{C}$, with an optimum at $50{ }^{\circ} \mathrm{C}$ (Figure $3 \mathrm{~B}$ ). At $60^{\circ} \mathrm{C}$, nitrilase activity was reduced to $30 \%$. Furthermore, stability of the nitrilase was tested over several weeks while the enzyme was stored at $4{ }^{\circ} \mathrm{C}$. Remaining catalytic activity $>80 \%$ could be observed during the first three weeks, followed by a slow decline (Figure $3 \mathrm{C}$ ). After three months, $8 \%$ of the initial activity remained. 

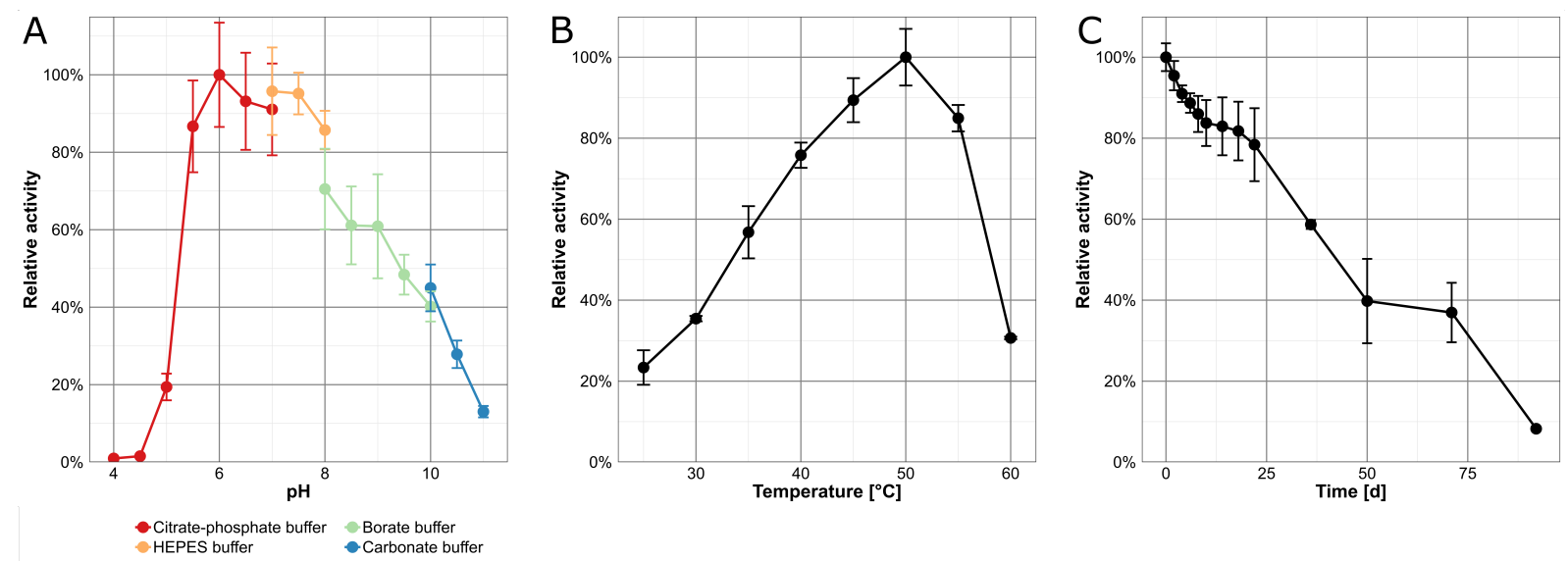

Figure 3: Temperature and pH dependence, and long-term stability of enzyme D3C71_601570. (A) pH optimum. Reactions were run for $2 \mathrm{~min}$ at $37^{\circ} \mathrm{C}$ in the respective buffers $(0.1 \mathrm{M})$ containing $1.25 \mu \mathrm{g}$ purified protein and $5 \mathrm{mM}$ phenylacetonitrile. $100 \%$ relative activity corresponds to $2.79 \mathrm{U} / \mathrm{mg}$. (B) Temperature optimum. Reactions were run for $2 \mathrm{~min}$ at various temperatures using citratephosphate buffer ( $\mathrm{pH} 6.0), 1.25 \mu \mathrm{g}$ purified protein, and $5 \mathrm{mM}$ phenylacetonitrile. $100 \%$ relative activity corresponds to $5.68 \mathrm{U} / \mathrm{mg}$. (C) Stability assay. Enzyme was stored at $4{ }^{\circ} \mathrm{C}$ in a buffered system ( $50 \mathrm{mM}$ monosodium phosphate, $300 \mathrm{mM}$ sodium chloride, $250 \mathrm{mM}$ imidazole, pH 8.0). Reactions were run for $2 \mathrm{~min}$ at $50{ }^{\circ} \mathrm{C}$ using citrate-phosphate buffer ( $\left.\mathrm{pH} 6.0\right), 1.25 \mu \mathrm{g}$ purified protein, and $5 \mathrm{mM}$ phenylacetonitrile. $100 \%$ relative activity corresponds to $5.68 \mathrm{U} / \mathrm{mg}$.

Catalytic activity of the nitrilase was tested with the substrates also used for the high-throughput assay, verifying its specificity for phenylacetonitrile under the tested conditions. To determine activity of the enzyme for structurally related compounds, four aromatic nitriles (2-phenylbutyronitrile, 3-phenylpropionitrile, benzonitrile, and mandelonitrile) were additionally tested. With 2-phenylbutyronitrile and benzonitrile as substrate, nitrilase activity was not detected but the enzyme exhibited reduced activity with mandelonitrile and 3-phenylpropionitrile (22 and $16 \%$ of activity measured with phenylacetonitrile, respectively) (Table 2). In conclusion, the metagenome-derived nitrilase D3C71_601570 is highly specific for phenylacetonitrile and belongs to the group of arylacetonitrilases.

To assess the effect of potential inhibitors and solvents on enzyme activity, the purified enzyme was tested against different chemicals. For detection of essential thiol residues, the thiol-binding reagents $\mathrm{HgCl}_{2}$ and $\mathrm{AgNO}_{3}$ were applied to the reaction mixture. The observed decline of enzyme activity to 1.6 and $2.0 \%$, respectively, indicated that the functionality of nitrilase D3C71_601570 depends on these residue (Table 3). As the metal-chelating agent EDTA had no effect on enzymatic function, dependence on metal-cofactors could be excluded. This was further supported by missing effects of divalent ions such as $\mathrm{Mg}^{2+}, \mathrm{Fe}^{2+}$, and $\mathrm{Mn}^{2+}$ on nitrilase activity. Interestingly, $\mathrm{Zn}^{2+}$ increased activity to $114 \%$. Most likely, stimulating effect of $\mathrm{Zn}^{2+}$ derives from a different mechanism, i.e. increased protein stability in the presence of $\mathrm{Zn}^{2+}$. Furthermore, treatment with $\mathrm{H}_{2} \mathrm{O}_{2}$ and SDS led to expected reduction of activity, which is most likely due to denaturation of the nitrilase. 
Strong inhibitory effects were encountered in the presence of DMSO, acetone, and chloroform even at $5 \%$ concentration (Table 4). At this concentration, nitrilase activity was less sensitive to methanol, ethanol, isopropanol, and glycerol treatment. At $20 \%$ concentration, only glycerol did not abolish the main enzymatic activity.

Table 2: Substrate specificity of enzyme D3C71_601570. Reactions were run for $15 \mathrm{~min}$ at $50^{\circ} \mathrm{C}$ using citrate-phosphate buffer $(\mathrm{pH} 6.0), 1.25 \mu \mathrm{g}$ purified protein, and $20 \mathrm{mM}$ nitrile. Activity with phenylacetonitrile as substrate $(5.19 \mathrm{U} / \mathrm{mg})$ was set as $100 \%$ relative activity.

\begin{tabular}{|l|l|}
\hline Substrate & Relative activity [\%] \\
\hline Phenylacetonitrile & $100.00 \pm 1.19$ \\
\hline Succinonitrile & $0.17 \pm 0.03$ \\
\hline Acetonitrile & $0.00 \pm 0.01$ \\
\hline Crotononitrile & $0.08 \pm 0.01$ \\
\hline 4-Hydroxybenzonitrile & $0 \pm 0.00$ \\
\hline Acetone cyanohydrin & $0.13 \pm 0.02$ \\
\hline Cyclohexanecarbonitrile & $0.09 \pm 0.01$ \\
\hline Fumaronitrile & $0.45 \pm 0.04$ \\
\hline 2-Phenylpropionitrile & $0.52 \pm 0.05$ \\
\hline Mandelonitrile & $22.01 \pm 0.61$ \\
\hline 2-Phenylbutyronitrile & $0.07 \pm 0.01$ \\
\hline 3-Phenylpropionitrile & $15.81 \pm 0.80$ \\
\hline Benzonitrile & $0.48 \pm 0.32$ \\
\hline
\end{tabular}

Table 3: Effects of various compounds on the activity of enzyme D3C71_601570. Reactions were run for $2 \mathrm{~min}$ at $50^{\circ} \mathrm{C}$ using citrate-phosphate buffer $(\mathrm{pH} 6.0), 1.25 \mu \mathrm{g}$ purified protein, $5 \mathrm{mM}$ phenylacetonitrile, and $1 \mathrm{mM}$ putative inhibitor. Enzyme activity with phenylacetonitrile as substrate without additions was set as $100 \%$ relative activity $(5.68 \mathrm{U} / \mathrm{mg})$.

\begin{tabular}{|c|c|}
\hline Compound & Relative activity [\%] \\
\hline EDTA & $104.45 \pm 1.62$ \\
\hline DTT & $76.21 \pm 3.29$ \\
\hline $\mathrm{H}_{2} \mathrm{O}_{2}$ & $24.56 \pm 2.35$ \\
\hline $\mathrm{HgCl}_{2}$ & $1.57 \pm 0.36$ \\
\hline $\mathrm{AgNO}_{3}$ & $1.96 \pm 0.18$ \\
\hline $\mathrm{CaCl}_{2}$ & $106.29 \pm 2.71$ \\
\hline $\mathrm{MnSO}_{4}$ & $98.01 \pm 3.80$ \\
\hline $\mathrm{MgSO}_{4}$ & $101.87 \pm 5.92$ \\
\hline $\mathrm{FeSO}_{4}$ & $98.25 \pm 5.65$ \\
\hline $\mathrm{CuSO}_{4}$ & $86.98 \pm 3.82$ \\
\hline $\mathrm{CoSO}_{4}$ & $81.01 \pm 2.60$ \\
\hline $\mathrm{ZnCl}_{2}$ & $114.00 \pm 2.04$ \\
\hline Sodium azide & $98.89 \pm 2.13$ \\
\hline SDS & $12.02 \pm 2.77$ \\
\hline
\end{tabular}


Table 4: Effect of different organic solvents on activity of enzyme D3C71_601570. Reactions were run for $2 \mathrm{~min}$ at $50{ }^{\circ} \mathrm{C}$ using citrate-phosphate buffer $(\mathrm{pH} 6.0), 1.25 \mu \mathrm{g}$ purified protein, and $5 \mathrm{mM}$ phenylacetonitrile. Enzyme activity with phenylacetonitrile as substrate without additions was set as $100 \%$ relative activity $(5.68 \mathrm{U} / \mathrm{mg})$.

\begin{tabular}{|l|l|l|}
\hline Solvent & $\begin{array}{l}\text { Relative activity of enzyme with } \mathbf{5 \%} \\
\text { v/v organic solvent [\%] }\end{array}$ & $\begin{array}{l}\text { Relative activity of enzyme with } \mathbf{2 0 \%} \\
\text { v/v organic solvent [\%] }\end{array}$ \\
\hline Methanol & $88.01 \pm 6.18$ & $9.26 \pm 1.97$ \\
\hline Ethanol & $85.22 \pm 7.12$ & $4.82 \pm 0.41$ \\
\hline Glycerol & $81.11 \pm 8.68$ & $76.65 \pm 9.06$ \\
\hline Isopropanol & $77.55 \pm 9.43$ & $3.63 \pm 0.91$ \\
\hline DMSO & $4.80 \pm 1.06$ & $2.32 \pm 0.72$ \\
\hline Acetone & $28.96 \pm 4.79$ & $0.14 \pm 0.07$ \\
\hline Chloroform & $24.81 \pm 3.44$ & $3.70 \pm 1.38$ \\
\hline Toluol & $8.12 \pm 2.13$ & $2.44 \pm 1.60$ \\
\hline
\end{tabular}

Finally, kinetic constants of nitrilase D3C71_601570 were determined. The reaction rate increased with substrate concentration until a plateau was reached at $5 \mathrm{mM}$. Between 10 and $50 \mathrm{mM}$ a rapid decrease in activity was recorded, followed by a slow decline until $100 \mathrm{mM}$ (Figure 4A), indicating inhibition by the substrate. Based on these data, kinetic constants were calculated with a non-linear model due to superior precision compared to linear methods like Lineweaver-Burk plots (19). Phenylacetonitrile was converted at $37^{\circ} \mathrm{C}$ with a $K_{\mathrm{M}}$ of $1.29 \mathrm{mM}$ and $V_{\max }$ of $13.85 \mathrm{U} / \mathrm{mg}$, reaching the saturation point at $6 \mathrm{mM}$ (Figure 4B). The enzyme is highly specific for this nitrile and the low $K_{\mathrm{M}}$ value indicated high affinity for the substrate.
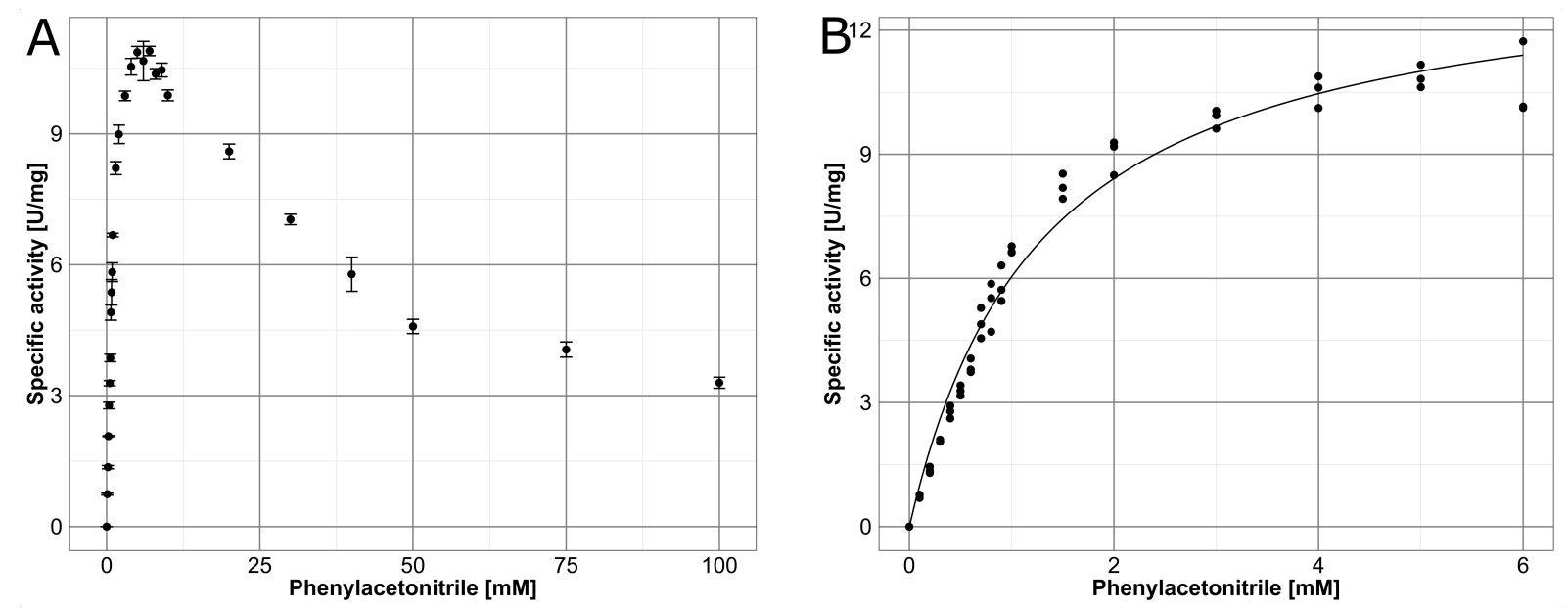

Figure 4: Influence of substrate concentrations on the reaction rate of enzyme D3C71_601570. Reactions were run with phenylacetonitrile as substrate at $37^{\circ} \mathrm{C}$ in a UV/Vis spectrophotometer. A: steady-state measurement until $100 \mathrm{mM}$ substrate concentration. B: steady-state measurement until saturation point at $6 \mathrm{mM}$ substrate concentration. C: Lineweaver-Burk plot. 


\section{DISCUSSION}

High-throughput nitrilase screening. In this study, a fast and simple high-throughput assay for screening of functional nitrilases was established. In comparison to the previously described method (15), it improves the total screening time by combining nitrilase reaction and subsequent ammonia conversion in a single reaction. Furthermore, it allows constant measurement of nitrile degradation and provides insights into the efficiency of the tested enzymes. Unfortunately, the measurement is limited by the GDH as only nitrilase reactions slower than the GDH can be monitored in detail. In addition, different enzymes in the crude extract can lead to ammonia release and influence the measurement. Other enzymes may also be involved in the reverse reaction of $N A D^{+}$to $N A D H$, leading to distorted results. To minimize the effect of these factors, additional precautions have to be taken. Most important is the use of crude extract containing the empty expression vector as negative control. To further reduce the likelihood of false positive hits, a time limit for nitrilase reaction can be used. Based on the reactions observed with the well-characterized $R$. rhodochrous K22 nitrilase $(16,17)$, this threshold was set at 15 minutes.

After establishment of screening parameters, 70 putative nitrilases identified in metagenomes of nitrile-treated microbial communities were taken as input. Combining the sequence-based nitrilase clusters obtained during bioinformatical analysis with results from the high-throughput assay, only candidates clustering with characterized nitrilases showed enzymatic activity. Skipping clusters consisting just of putative nitrilases during future screenings would reduce subsequent work but involves the risk of missing nitrilases with novel sequences. Therefore, decisions on the cost-benefit ratio of including these clusters in further analyses must be individually made.

Although only preselected putative nitrilase genes were amplified, not all of them could be overexpressed or the gene products formed inclusion bodies in E. coli. These results could be due to different codon usage of $E$. coli TOP10 compared to the native hosts of the enzymes. Diverging rare codons in E. coli could lead to suboptimal expression and low amounts of the desired protein or unwanted mRNA degradation (20-23). Furthermore, misfolding and aggregation due to missing translational pauses could occur (24). One possibility to avoid these problems is using lower temperatures during protein expression or the utilization of low-copy-number plasmids (25-28). In addition, codon-optimization of the candidate genes for $E$. coli could be performed. However, these methods were not applied as they are mostly not suitable for large numbers of candidates.

Characterization of an arylacetonitrilase. The prediction and subsequent isolation of a phenylacetonitrile-degrading nitrilase shows the reliability of the novel nitrilase high-throughput screening. According to sequence similarity, the isolated arylacetonitrilase is affiliated to Variovorax 
boronicumulans (Table 1). Nitrile-degrading abilities of this species have been described before, but phenylacetonitrile degradation has not been mentioned (29-31).

With $36 \mathrm{kDa}$ the here described enzyme is close to molecular mass of other known phenylacetonitriledegrading nitrilases such as the nitrilases from Synechocystis sp. PCC 6803 (40 kDa), Bradyrhizobium japonicum USDA 110 (34.5 kDa), or Alcaligenes faecalis ATCC 8750 (36 kDa) (32-34). In contrast, the broad $\mathrm{pH}$ range of D3C71_601570 activity is not that common $(34,35)$. In addition, it exhibits an unusual long-term stability, as similar nitrilases loose up to $80 \%$ activity in ten days $(32,35)$, whereas the here characterized enzyme still retained $80 \%$ activity after three weeks.

One major difference of the here presented enzyme to all other phenylacetonitrile-degrading nitrilases is its narrow substrate range. Phenylacetonitrile was the preferred substrate and even small differences in molecular structure seem to inhibit proper catalytic function. During high-throughput assay, minor degradation of other nitriles was observed in the crude extract of the strain producing enzyme D3C71_601570. None of these substrates were degraded by the purified enzyme, underlining again the importance of the 15-minute threshold to avoid identification of false positive candidates. Nevertheless, the differences can also come from the His 6 -tag added for purification $(36,37)$.

\section{CONCLUSION}

In this study, we could show that a combination of targeted sequence data analyses with a highthroughput activity assay can assist in the fast identification of candidates for functional nitrilases. Due to real-time measurement of NADH degradation, the here presented assay does not only provide hints on possible substrates but also on the catalytic efficiency of tested enzymes. Furthermore, the use of crude extract is less time consuming compared to methods demanding purified enzymes.

One of the active enzymes showed phenylacetonitrile-degrading abilities. The most likely to Variovorax belonging nitrilase is highly specific for this arylacetonitrile, as closely related molecules were barely degraded. To our knowledge, the enzyme shows unique properties, as other nitrilases usually degrade several (related) substrates. Therefore, it can give new insights into sequence function relationship of arylacetonitrilases and help to develop better biocatalysts.

\section{MATERIAL AND METHODS}

Metagenomic DNA and putative nitrilases. Metagenomic DNA originated from soil enrichment cultures containing either phenylacetonitrile, succinonitrile, acetonitrile, crotononitrile, 4-hydroxybenzonitrile, or cyclohexanecarbonitrile (Chapter 3.1). Genbank accession numbers of the annotated metagenomes are as follows: phenylacetonitrile, RCUE00000000; succinonitrile, RCUP00000000; acetonitrile, RCUQ00000000, crotononitrile, RCUN00000000; 4-hydroxybenzonitrile, RCUF00000000; cyclohexanecarbonitrile, RCU000000000. 
Bioinformatic analysis. Nitrilase reference data were obtained from the SWISS-Prot database (38) (date of search: December 12th, 2017). The data were further processed by removing misannotated enzymes, mere subunits, or nitrile hydratases designated as nitrilases. Clustering of data was done with the web suite CD-HIT (39). A fasta file containing the SWISS-Prot nitrilases and the putative nitrilases was used as input and a sequence identity cut-off of $40 \%$ was set for clustering.

Nitrilase positive control. An already characterized aliphatic nitrilase from Rhodococcus rhodochrous K22 was used as positive control for establishing the high-throughput nitrilase assay $(16,17)$. The nitrilase was codon-optimized for Escherichia coli K12 derivates using the web suite JCat (http://www.jcat.de) (40) and synthesized by Integrated DNA Technologies (Leuven, Belgium).

Media. LB-Miller (10 g tryptone, $10 \mathrm{~g} \mathrm{NaCl}$, and $5 \mathrm{~g}$ yeast extract per liter) (41) was used for growth of microorganisms. For agar plates, $15 \mathrm{~g}$ agar per liter was added. Media were sterilized by autoclaving at $121^{\circ} \mathrm{C}$ for $20 \mathrm{~min}$.

PCR protocol. $50 \mu$ I PCR reaction mixture contained $10 \mu$ l 5-fold Phusion HF buffer, $200 \mu \mathrm{M}$ of each dNTP, $0.2 \mu \mathrm{M}$ of both primers (Data Set S3), 3\% DMSO, 50 ng metagenomic DNA as template, $1 \mathrm{U}$ of Phusion polymerase (Thermo Fisher Scientific, Waltham, MA, USA) and was filled up with $\mathrm{ddH}_{2} \mathrm{O}$. Initial denaturation was done at $98^{\circ} \mathrm{C}$ for $5 \mathrm{~min}$, followed by 30 cycles of denaturation $\left(98^{\circ} \mathrm{C}, 30 \mathrm{~s}\right)$, annealing (temperature based on primer properties, $30 \mathrm{~s}$ ) and elongation $\left(72{ }^{\circ} \mathrm{C}, 45 \mathrm{~s} / \mathrm{kbp}\right)$. The final elongation was for 5 min at $72{ }^{\circ} \mathrm{C}$.

Plasmids. Amplified putative nitrilase-encoding genes (Data Set S2) were sub-cloned into the pBAD18 vector system (42). For selection, a final concentration of $100 \mu \mathrm{g} / \mathrm{ml}$ ampicillin was used. The fidelity of the constructs was checked by sequencing (Microsynth Seqlab, Göttingen, Germany) with forward primer 5'-ATTAGCGGATCCTACCTGACG-3' and reverse primer 5'-CAGACCGCTTCTGCGTTCTG-3'.

Strains and transformation. Chemically competent Escherichia coli TOP10 cells (Thermo Fisher Scientific, Waltham, MA, USA) were transformed according to the manufacturer's protocol. Subsequently, cells were plated on ampicillin-containing LB plates and incubated overnight at $37^{\circ} \mathrm{C}$.

Heterologous expression. E. coli TOP10 containing the pBAD18 vector with desired insert was grown in LB medium at $37^{\circ} \mathrm{C}$ overnight at $180 \mathrm{rpm}$ (Innova 44 shaker, New Brunswick Scientific, Nürtingen, Germany). The preculture was used to inoculate $10 \mathrm{ml} \mathrm{LB}$ medium to an $\mathrm{OD}_{600}$ of 0.1 , which was subsequently incubated for $1.5 \mathrm{~h}$ at $37^{\circ} \mathrm{C}$ and $180 \mathrm{rpm}$ (Innova 44 shaker) to reach an $\mathrm{OD}_{600}$ of $0.6-$ 0.8. For induction of expression, $1.5 \% \mathrm{~L}-(+)$-arabinose were added, followed by $6 \mathrm{~h}$ of incubation. 
Cell disruption and purification of $\mathrm{His}_{6}$-tagged protein. Cells containing the overexpressed (His $_{6}{ }^{-}$ tagged) nitrilase were washed twice with 1x LEW buffer from the Protino Ni-TED kit (Macherey-Nagel, Düren, Germany) and resuspended in $1.5 \mathrm{ml}$ buffer with $40 \mu \mathrm{g} / \mathrm{ml}$ DNase I and $0.1 \% \mathrm{w} / \mathrm{v}$ lysozyme. The cells were then disrupted at least three times by French press at $1.38 \times 10^{8} \mathrm{~Pa}$ (Thermo Fisher Scientific). The soluble fractions of the thereby recovered solution were obtained by centrifugation at $6000 \times \mathrm{g}$ and $4{ }^{\circ} \mathrm{C}$ for $20 \mathrm{~min}$ to remove cell debris. For purification, the resulting supernatant was then loaded according to the manufacturer's protocol. The purified enzyme was further analyzed by $12 \%$ SDS-PAGE and protein concentration was determined using Bradford method (43) with bovine serum albumin as standard.

Nitriles. Nitriles were solved at a concentration of 2.7 M in DMF (Merck KGaA, Darmstadt, Germany). These stock solutions were filtered sterile and stored at $4{ }^{\circ} \mathrm{C}$. Following nitriles were used: phenylacetonitrile, acetonitrile (all $\mathrm{TCl}$ Deutschland $\mathrm{GmbH}$, Eschborn, Germany), succinonitrile, crotonitrile, 4-hydroxybenzonitrile, acetone cyanohydrin, cyclohexanecarbonitrile, fumaronitrile, and 2-phenylpropionitrile (all Sigma-Aldrich Chemie GmbH, Munich, Germany). In addition, mandelonitrile, 2-phenylbutyronitrile, benzonitrile (all Sigma-Aldrich), and 3-phenylpropiontrile (Alfa Aesar, Haverhill, MA, USA) were used for characterization of the arylacetonitrilase.

High-throughput nitrilase assay. Degradation of nitriles was measured by monitoring the release of ammonia in a coupled enzymatic reaction (44). The ammonia reacts with NADH and $\alpha$-ketoglutarate through a glutamate dehydrogenase $(\mathrm{GDH})$ to glutamate. The reduced cofactor is monitored by its absorbance change at $340 \mathrm{~nm}$. For screening of putative nitrilases, the high-throughput assay of Vergne-Vaxelaire et al. (15) was adjusted to allow real-time measurements without time-consuming incubation steps (15). The assay was performed with technical replicates in a flat-based 96-well plate. The reaction mixture (final volume $250 \mu \mathrm{l}$ ) contained $0.5 \mathrm{mM} \mathrm{NADH}, 1 \mathrm{mM} \alpha$-ketoglutarate, $37 \mu \mathrm{l}$ of a 2.7 $\mathrm{M}$ nitrile stock solution (solved in DMF), $20 \mu \mathrm{g}$ crude extract (added at the end), and $1 \mathrm{U} / \mathrm{ml} \mathrm{GDH}$ from bovine liver type II (Sigma-Aldrich Chemie $\mathrm{GmbH}$ ) and $50 \mathrm{mM}$ Tris- $\mathrm{HCl}(\mathrm{pH} \mathrm{8.0)}$ buffer. As negative control, crude extract containing the pBAD18 cloning vector was used. The reaction mixture was incubated at $37^{\circ} \mathrm{C}$ in a Synergy 2 microplate reader (BioTek Instruments $\mathrm{GmbH}$, Bad Friedrichshall, Germany). The absorbance was constantly measured for $1.5 \mathrm{~h}$ at $340 \mathrm{~nm}$. For calculation, the values of the pBAD18 crude extract (negative control) were subtracted from that of the nitrilase crude extract. To avoid identification of false positives, only reactions completed during the first 15 minutes of measurement were considered as nitrilase activity. 
Nitrilase characterization. The selected nitrilase (NCBI accession D3C71_601570) was characterized using a colorimetric assay for detection of ammonia (45). Nitrilase reaction was performed in a $500 \mu \mathrm{l}$ mixture containing $25 \mu \mathrm{l}$ nitrile (100 mM, solved in DMF), $1.25 \mu \mathrm{g}$ purified His 6 -tagged nitrilase and $0.1 \mathrm{M}$ citrate-phosphate buffer $(\mathrm{pH} 6.0)$ for $2 \mathrm{~min}$ at $50^{\circ} \mathrm{C}$. The experimental environment was at $23^{\circ} \mathrm{C}$. $111 \mu \mathrm{l}$ of this reaction were transferred into $222 \mu \mathrm{l}$ sodium phenate, followed by $333 \mu \mathrm{l}$ of $0.01 \%$ sodium nitroprusside and $333 \mu \mathrm{l}$ of hypochlorite. The reaction mixture was incubated for $15 \mathrm{~min}$ at $27^{\circ} \mathrm{C}$ in darkness and subsequently absorption was measured at $630 \mathrm{~nm}$. Same procedure was used for substrate determination with $20 \mathrm{mM}$ of the respective nitrile and $15 \mathrm{~min}$ incubation.

Nitrilase inhibitors. Effect of various compounds on nitrilase activity was tested. Incubation was carried out at $50^{\circ} \mathrm{C}$ for $2 \mathrm{~min}$ in the standard reaction mixture containing a putative inhibitor at $1 \mathrm{mM}$ or $5 \% \mathrm{v} / \mathrm{v}$ and $20 \% \mathrm{v} / \mathrm{v}$ when testing organic solvents.

Nitrilase stability. For stability testing, the $\mathrm{His}_{6}$-tagged purified enzyme was stored in Protino ${ }^{\circledR} \mathrm{Ni}$-TED elution buffer (Macherey-Nagel, Düren, Germany) containing $1 \mathrm{mM}$ sodium azid at $4{ }^{\circ} \mathrm{C}$ in the dark.

Steady-state activity assay. The steady-state kinetics for arylacetonitrilase activities were measured using a GDH (bovine liver type II) to detect the concomitant oxidation of NADH at $340 \mathrm{~nm}$ and $37^{\circ} \mathrm{C}$ using a UV/Vis spectrophotometer (Cary 100 UV-Vis, Varian Medical Systems, Darmstadt, Germany). The reaction mixture contained $0.5 \mathrm{mM} \mathrm{NADH}, 1 \mathrm{mM} \alpha$-ketoglutarate, $0.1 \mathrm{mM}$ to $100 \mathrm{mM}$ of phenylacetonitrile (final volume $37 \mu \mathrm{l}$, nitrile solved in DMF), $5 \mu \mathrm{g}$ purified nitrilase (added at the end), and $1 \mathrm{U} / \mathrm{ml} \mathrm{GDH}$ from bovine liver type II (Sigma-Aldrich Chemie $\mathrm{GmbH}$ ) filled up to $1000 \mu$ l with $0.1 \mathrm{M}$ HEPES ( $\mathrm{pH} 8.0$ ) buffer. Negative control did not contain the nitrilase. The slope $\left(\Delta \mathrm{A}_{340}\right)$ was determined by Cary WinUV version 3.0 (Varian Medical Systems). The $k_{\mathrm{cat}}$ values were plotted against phenylacetonitrile concentrations. Further, the macroscopic constants $k_{\mathrm{cat}}$ and $K_{\mathrm{M}}$ were analyzed using the Michaelis-Menten equation of R package 'drc' (46).

\section{FUNDING INFORMATION}

The work of R.E. was supported by the "Fonds der Chemischen Industrie im Verband der Chemischen Industrie e.V.". The funders had no role in study design, data collection, and interpretation, or the decision to submit the work for publication. 


\section{REFERENCES}

1. Conn EE. 1979. Biosynthesis of cyanogenic glycosides. Naturwissenschaften 66:28-34.

2. Jallageas J-C, Arnaud A, Galzy P. 1980. Bioconversion of nitriles and their applications., p. 1-32. In Fiechter, A (ed.), Advances in biochemical engineering, 1st ed. Springer Berlin Heidelberg, Berlin, Germany.

3. Martínková L, Uhnáková B, Pátek M, Nešvera J, Křen V. 2009. Biodegradation potential of the genus Rhodococcus. Environ Int 35:162-177.

4. Rustler S, Stolz A. 2007. Isolation and characterization of a nitrile hydrolysing acidotolerant black yeast - Exophiala oligosperma R1. Appl Microbiol Biotechnol 75:899-908.

5. Piotrowski M. 2008. Primary or secondary? Versatile nitrilases in plant metabolism. Phytochemistry 69:2655-2667.

6. Shaw NM, Robins KT, Kiener A. 2003. Lonza: 20 years of biotransformations. Adv Synth Catal 345:425-435.

7. Panova A, Mersinger $L$, Liu Q, Foo T, Roe DC, Spillan WL, Sigmund AE, Ben-Bassat A, Wagner LW, O'Keefe DP, Wu S, Petrillo KL, Payne MS, Breske ST, Gallagher FG, DiCosimo R. 2007. Chemoenzymatic synthesis of glycolic acid. Adv Synth Catal 349:1462-1474.

8. Bui K, Maestracci M, Thiery A, Arnaud A, Galzy P. 1984. A note on the enzymic action and biosynthesis of a nitrile-hydratase from a Brevibacterium sp. J Appl Bacteriol 57:183-190.

9. Vaughan PA, Knowles CJ, Cheetham PSJ. 1989. Conversion of 3-cyanopyridine to nicotinic acid by Nocardia rhodochrous LL100-21. Enzyme Microb Technol 11:815-823.

10. Almatawah QA, Cowan DA. 1999. Thermostable nitrilase catalysed production of nicotinic acid from 3-cyanopyridine. Enzyme Microb Technol 25:718-724.

11. Zhang Z-J, Pan J, Liu J-F, Xu J-H, He Y-C, Liu Y-Y. 2011. Significant enhancement of (R)-mandelic acid production by relieving substrate inhibition of recombinant nitrilase in toluene-water biphasic system. J Biotechnol 152:24-29.

12. Robertson DE, Chaplin J, DeSantis G, Podar M, Madden M, Chi E, Richardson T, Milan A, Miller M, Weiner DP, Wong K, McQuaid J, Farwell B, Preston L, Tan X, Snead M, Keller M, Mathur E, Kretz PL, Burk MJ, Short JM. 2004. Exploring nitrilase sequence space for enantioselective catalysis. Appl Environ Microbiol 70:2429-2436.

13. Bayer S, Birkemeyer C, Ballschmiter M. 2011. A nitrilase from a metagenomic library acts regioselectively on aliphatic dinitriles. Appl Microbiol Biotechnol 89:91-98.

14. Soares Bragança CR, Dooley-Cullinane T-M, O’Reilly C, Coffey L. 2017. Applying functional metagenomics to search for novel nitrile-hydrolyzing enzymes using environmental samples. Biomater Tissue Technol 1:1000108.

15. Vergne-Vaxelaire C, Bordier F, Fossey A, Besnard-Gonnet M, Debard A, Mariage A, Pellouin V, Perret A, Petit J-L, Stam M, Salanoubat M, Weissenbach J, De Berardinis V, Zaparucha A. 2013. Nitrilase activity screening on structurally diverse substrates: Providing biocatalytic tools for organic synthesis. Adv Synth Catal 355:1763-1779. 
16. Kobayashi M, Yanaka N, Nagasawa T, Yamada H. 1990. Purification and characterization of a novel nitrilase of Rhodococcus rhodochrous $\mathrm{K} 22$ that acts on aliphatic nitriles. J Bacteriol 172:4807-4815.

17. Kobayashi M, Yanaka N, Nagasawa T, Yamada H. 1992. Primary structure of an aliphatic nitriledegrading enzyme, aliphatic nitrilase, from Rhodococcus rhodochrous $\mathrm{K} 22$ and expression of its gene and identification of its active site residue. Biochemistry 31:9000-9007.

18. Mueller P, Egorova K, Vorgias CE, Boutou E, Trauthwein H, Verseck S, Antranikian G. 2006. Cloning, overexpression, and characterization of a thermoactive nitrilase from the hyperthermophilic archaeon Pyrococcus abyssi. Protein Expr Purif 47:672-681.

19. Cho Y-S, Lim H-S. 2018. Comparison of various estimation methods for the parameters of Michaelis-Menten equation based on in vitro elimination kinetic simulation data. Transl Clin Pharmacol 26:39.

20. Chen G-FF, Inouye M. 1990. Suppression of the negative effect of minor arginine codons on gene expression; preferential usage of minor codons within the first 25 codons of the Escherichia coli genes. Nucleic Acids Res 18:1465-1473.

21. Kane JF. 1995. Effects of rare codon clusters on high-level expression of heterologous proteins in Escherichia coli. Curr Opin Biotechnol 6:494-500.

22. Gustafsson C, Govindarajan S, Minshull J. 2004. Codon bias and heterologous protein expression. Trends Biotechnol 22:346-353.

23. Presnyak V, Alhusaini N, Chen Y, Martin S, Morris N, Kline N, Olson S, Weinberg D, Baker KE, Graveley BR, Coller J. 2015. Codon optimality is a major determinant of mRNA stability. Cell 160:1111-1124.

24. Rosano GL, Ceccarelli EA. 2009. Rare codon content affects the solubility of recombinant proteins in a codon bias-adjusted Escherichia coli strain. Microb Cell Fact 8:41.

25. Schein C, Noteborn MHM. 1988. Formation of soluble recombinant proteins in Escherichia coli is favored by lower growth temperature. Nat Biotechnol 6:709-712.

26. Vera A, González-Montalbán N, Arís A, Villaverde A. 2007. The conformational quality of insoluble recombinant proteins is enhanced at low growth temperatures. Biotechnol Bioeng 96:1101-1106.

27. San-Miguel T, Pérez-Bermúdez P, Gavidia I. 2013. Production of soluble eukaryotic recombinant proteins in $E$. coli is favoured in early log-phase cultures induced at low temperature. Springerplus 2:89.

28. Na G, Wolfe A, Ko C, Youn H, Lee YM, Byun SJ, Jeon I, Koo Y. 2013. A low-copy-number plasmid for retrieval of toxic genes from BACs and generation of conditional targeting constructs. Mol Biotechnol 54:504-514.

29. Zhang H-J, Zhou Q-W, Zhou G-C, Cao Y-M, Dai Y-J, Ji W-W, Shang G-D, Yuan S. 2012. Biotransformation of the neonicotinoid insecticide thiacloprid by the bacterium Variovorax boronicumulans strain $\mathrm{J} 1$ and mediation of the major metabolic pathway by nitrile hydratase. $\mathrm{J}$ Agric Food Chem 60:153-159. 
30. Egelkamp R, Schneider D, Hertel R, Daniel R. 2017. Nitrile-degrading bacteria isolated from compost. Front Environ Sci 5:56.

31. Sun S-L, Yang W-L, Fang W-W, Zhao Y-X, Guo L, Dai Y-J. 2018. The plant growth-promoting rhizobacterium Variovorax boronicumulans CGMCC 4969 regulates the level of indole-3-acetic acid synthesized from indole-3-acetonitrile. Appl Environ Microbiol 84:e00298-18.

32. Heinemann U, Engels D, Bürger S, Kiziak C, Mattes R, Stolz A. 2003. Cloning of a nitrilase gene from the cyanobacterium Synechocystis sp. strain PCC6803 and heterologous expression and characterization of the encoded protein. Appl Environ Microbiol 69:4359-4366.

33. Zhu D, Mukherjee C, Yang Y, Rios BE, Gallagher DT, Smith NN, Biehl ER, Hua L. 2008. A new nitrilase from Bradyrhizobium japonicum USDA 110. Gene cloning, biochemical characterization and substrate specificity. J Biotechnol 133:327-333.

34. Yamamoto K, Fujimatsu I, Komatsu KI. 1992. Purification and characterization of the nitrilase from Alcaligenes faecalis ATCC 8750 responsible for enantioselective hydrolysis of mandelonitrile. J Ferment Bioeng 73:425-430.

35. Nagasawa T, Mauger J, Yamada H. 1990. A novel nitrilase, arylacetonitrilase, of Alcaligenes faecalis JM3. Purification and characterization. Eur J Biochem 194:765-772.

36. Lee YL, Su MS, Huang TH, Shaw JF. 1999. C-terminal His-tagging results in substrate specificity changes of the thioesterase I from Escherichia coli. J Am Oil Chem Soc 76:1113-1118.

37. Majorek KA, Kuhn ML, Chruszcz M, Anderson WF, Minor W. 2014. Double trouble - Buffer selection and His-tag presence may be responsible for nonreproducibility of biomedical experiments. Protein Sci 23:1359-1368.

38. Bairoch A, Apweiler R. 2000. The SWISS-PROT protein sequence database and its supplement TrEMBL in 2000. Nucleic Acids Res 28:45-48.

39. Huang Y, Niu B, Gao Y, Fu L, Li W. 2010. CD-HIT Suite: A web server for clustering and comparing biological sequences. Bioinformatics 26:680-682.

40. Grote A, Hiller K, Scheer M, Münch R, Nörtemann B, Hempel DC, Jahn D. 2005. JCat: a novel tool to adapt codon usage of a target gene to its potential expression host. Nucleic Acids Res 33:W526-31.

41. Miller JH. 1972. Experiments in molecular genetics., 1st ed. Cold Spring Harbor Laboratory, Cold Spring Harbor, NY, USA.

42. Guzman L-M, Belin D, Carson MJ, Beckwith J. 1995. Tight regulation, modulation, and high-level expression by vectors containing the arabinose PBAD promoter. J Bacteriol 177:4121-4130.

43. Bradford MM. 1976. A rapid and sensitive method for the quantitation of microgram quantities of protein utilizing the principle of protein-dye binding. Anal Biochem 72:248-254.

44. Reisinger C, van Assema F, Schürmann M, Hussain Z, Remler P, Schwab H. 2006. A versatile colony assay based on NADH fluorescence. J Mol Catal B Enzym 39:149-155.

45. Fawcett JK, Scott JE. 1960. A rapid and precise method for the determination of urea. J Clin Pathol 13:156-159. 
46. Ritz C, Baty F, Streibig JC, Gerhard D. 2015. Dose-response analysis using R. PLoS One 10:e0146021. 


\subsection{Novel approach for identification of biocatalysts}

Richard Egelkamp ${ }^{1}$, Heiner Klingenberg ${ }^{2}$, Robert Hertel ${ }^{1}$ \& Rolf Daniel ${ }^{1}$

${ }^{1}$ Genomic and Applied Microbiology \& Göttingen Genomics Laboratory, Institute of Microbiology and Genetics, University of Göttingen, Göttingen, Germany

${ }^{2}$ Bioinformatics,

Institute of Microbiology and Genetics, University of Göttingen, Göttingen, Germany

Supplementary data can be found on the enclosed DVD

$\underline{\text { Author contributions }}$

Performed the experiments: $\mathbf{R E}$

Analyzed the data: RE, HK

Wrote the publication: $\mathbf{R E}, \mathrm{RH}, \mathrm{RD}$

Conceived the study: RE, RH, RD 


\section{INTRODUCTION}

Molecules containing a $-\mathrm{C} \equiv \mathrm{N}$ functional group are known as nitriles. These structurally divers and often toxic compounds are widespread in nature and present mainly in plants (1). Enzymatic degradation of nitriles is performed by two different known pathways: the direct degradation of nitriles to corresponding carboxylic acids and ammonia by nitrilases, or the hydration of nitriles to amides by nitrile hydratases (NHases) and the subsequent degradation to carboxylic acids and ammonia by amidases. In addition to their natural occurrence, nitriles are also an important compound in industry for the production of different bulk chemicals and pharmaceuticals $(2-5)$. Due to the increasing interest in green chemistry and therefore a high demand on nitrile-degrading enzymes, identification of novel nitrilases is of increasing importance (6).

In general, screening for novel enzymes by (meta)genomic approaches is done employing two methods. The first is a function-based screening using (meta)genomic libraries and their expression in heterologous hosts like Escherichia coli (7). This method could lead to the discovery of genes encoding unknown enzymes but requires high effort. Furthermore, heterologous protein expression could lead to non-functional proteins and therefore prevent their detection. The second approach is a sequencebased approach in which (meta)genomic datasets are screened for genes of interest (8). This method is limited to homologues of already known enzymes, making the detection of truly novel proteins impossible. In addition, genes merely identified by sequence similarities do not necessarily exhibit the targeted function.

During the last years, a third screening approach based on differential transcriptomics arose (9-11). For this purpose, an organism is treated under at least two different conditions and respective transcriptomes are sequenced. By statistical analysis, up- and downregulated genes of each treatment are identified, providing insight into genes important for specific conditions. Drawback of this method are the complex models for normalization and the high computational power needed for analysis $(12,13)$.

In this study, an experimental setup for the (meta)transcriptome-based discovery of genes encoding nitrile-degrading enzymes is presented. For microbial growth, media with acetonitrile and for comparison with acetic acid and ammonia as sole carbon and nitrogen sources were used. Therefore, genes upregulated in acetonitrile-treated cultures compared to acetic acid-containing samples are promising candidates for novel nitrile-degrading enzymes. In addition, unknown transporters or resistance mechanisms can be identified by this method. In a previous experiment, Rhodococcus erythropolis ACN1 was isolated from compost and its acetonitrile-degrading ability experimentally confirmed (14). The isolate was used during the here presented study to establish growth conditions 
and bioinformatic analysis strategies. Subsequently, the approach was applied to a complex microbial community for identification of genes encoding novel nitrilases by differential metatranscriptomics.

\section{RESULTS}

Establishment of a transcriptome-based nitrilase screening. R. erythropolis ACN1 was grown in minimal medium containing acetic acid and ammonia (ACA) or acetonitrile (ACN) as sole carbon and nitrogen sources. Growth of the ACN cultures was delayed by approx. 25 hours when compared with ACA samples. After initial lag phase, similar growth pattern was observed (Figure 1). Final optical density $\left(\mathrm{OD}_{600}\right)$ of the $\mathrm{ACA}$ and the $\mathrm{ACN}$ cultures were almost identical (1.6 and 1.5, respectively).

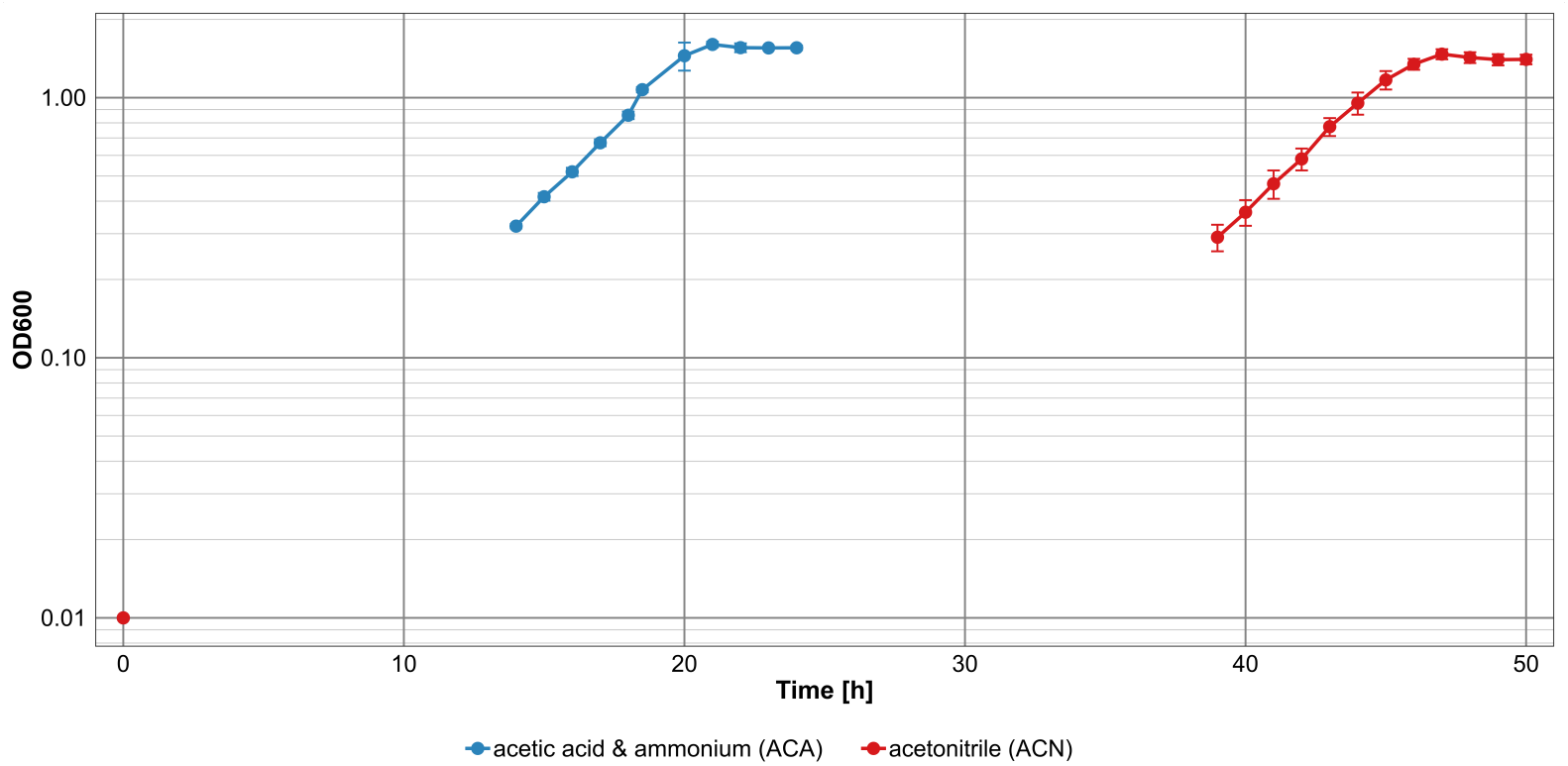

Figure 1: Growth of $R$. erythropolis ACN1 under different treatments.

For both conditions, transcriptomes were harvested during logarithmic growth. After quality-filtering, lowest number of transcriptome reads was 17.8 million and more than $99 \%$ of the filtered reads mapped onto the genomic sequence of $R$. erythropolis ACN1 (14). Subsequent normalization and analysis were performed with DESeq2, an algorithm optimized for transcriptome comparison (15). The algorithm offers a false discovery rate (FDR) cutoff to reduce number of erroneously identified differentially expressed genes. To minimize number of false positives, a cutoff of 0.025 was chosen for further analysis. With this setting, 186 differentially expressed genes were identified of which 26 were upregulated and 160 downregulated in the ACN culture when compared to the ACA treatment.

Besides this statistical parameter, the difference between both conditions considered as significantly up- or downregulated was defined. For that purpose, a logarithmic fold change $\left(\log _{2} \mathrm{FC}\right)$ between the normalized gene read counts was calculated. As a $\log _{2} \mathrm{FC}$ of 2.0 is commonly used and streamlines subsequent analysis (16-18), respective values were applied in this study. This second criterium reduced the number of differentially expressed candidate genes to 23 of which 4 were upregulated 
and 19 downregulated in the ACN culture (Table 1). Further analysis focused on upregulated genes, as they are most promising to comprise the targeted genes encoding nitrile-degrading enzymes.

The upregulated genes belonged to two groups. The first group contained an amidase (BKP42_54900) with a $\log _{2} \mathrm{FC}$ of 2.1, while the second group consisted of the remaining upregulated genes and was in proximity to the amidase. All members were upregulated at least six-fold, indicating a strong effect of the nitrile on their expression. Nevertheless, none of the respective annotations indicated a nitrilerelated function, as they consisted of a tubulin-like protein (BKP42_54850), a hypothetical protein (BKP42_54860), and a GTPase domain (BKP42_54870). When performing additional blastn and blastp searches against the NCBI nr database, annotation of the aliphatic amidase was confirmed, whereas best hits for the remaining upregulated genes always consisted of hypothetical proteins (Table 2). An additional motif search against the NCBI CDD (conserved domain database) supported annotation of the amidase and the tubulin family GTPase.

Table 1: Significantly up- or downregulated genes in R. erythropolis ACN1 when grown with acetonitrile compared to treatment with acetic acid and ammonia. Differential expression was analyzed with DESeq2 with an FDR of 0.025 and $\log _{2} \mathrm{FC}$ of $\geq 2$ as cutoff. Positive values (bold) represent genes upregulated under $\mathrm{ACN}$ treatment; negative values represent downregulated genes.

\begin{tabular}{|l|l|l|}
\hline Locustag & Gene & log $_{2}$ FC \\
\hline BKP42_13460 & CAAX amino terminal protease self- immunity & -2.0 \\
\hline BKP42_14580 & putative siderophore transport system permease protein YfiZ precursor & -2.2 \\
\hline BKP42_22340 & hypothetical protein & -2.2 \\
\hline BKP42_29290 & Enterobactin exporter EntS & -2.3 \\
\hline BKP42_29300 & Ferrienterobactin-binding periplasmic protein precursor & -2.3 \\
\hline BKP42_29310 & Linear gramicidin synthase subunit B & -2.6 \\
\hline BKP42_29320 & Flavodoxin & -2.3 \\
\hline BKP42_29330 & Isochorismatase & -2.6 \\
\hline BKP42_29340 & 2,3-dihydroxybenzoate-AMP ligase & -2.4 \\
\hline BKP42_29350 & 2,3-dihydro-2,3-dihydroxybenzoate dehydrogenase & -2.0 \\
\hline BKP42_29370 & Isochorismate synthase DhbC & -2.0 \\
\hline BKP42_29390 & hypothetical protein & -3.3 \\
\hline BKP42_39400 & Ferrienterobactin-binding periplasmic protein precursor & -3.4 \\
\hline BKP42_39410 & Enterochelin esterase & -2.3 \\
\hline BKP42_43800 & putative siderophore transport system permease & -2.0 \\
\hline BKP42_43810 & Ferric enterobactin transport system permease protein FepG & -2.7 \\
\hline BKP42_54850 & Tubulin-like protein CetZ & $\mathbf{3 . 2}$ \\
\hline BKP42_54860 & hypothetical protein & $\mathbf{3 . 9}$ \\
\hline BKP42_54870 & Tubulin/FtsZ family, GTPase domain & $\mathbf{3 . 9}$ \\
\hline BKP42_54900 & Aliphatic amidase & $\mathbf{2 . 1}$ \\
\hline BKP42_65440 & Fe(3+)-citrate-binding protein YfmC precursor & -2.1 \\
\hline BKP42_65450 & Iron(3+)-hydroxamate-binding protein FhuD precursor & -2.0 \\
\hline BKP42_65460 & putative siderophore transport system ATP-binding protein YusV & -2.1 \\
\hline & & \\
\hline
\end{tabular}




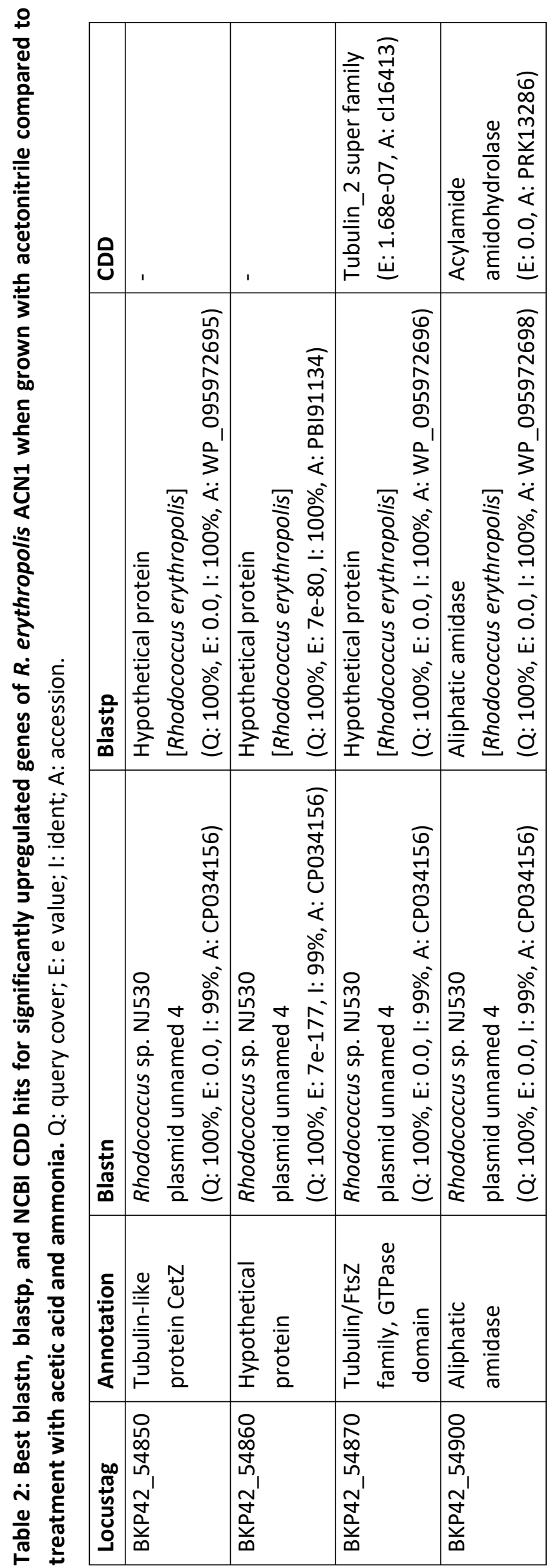




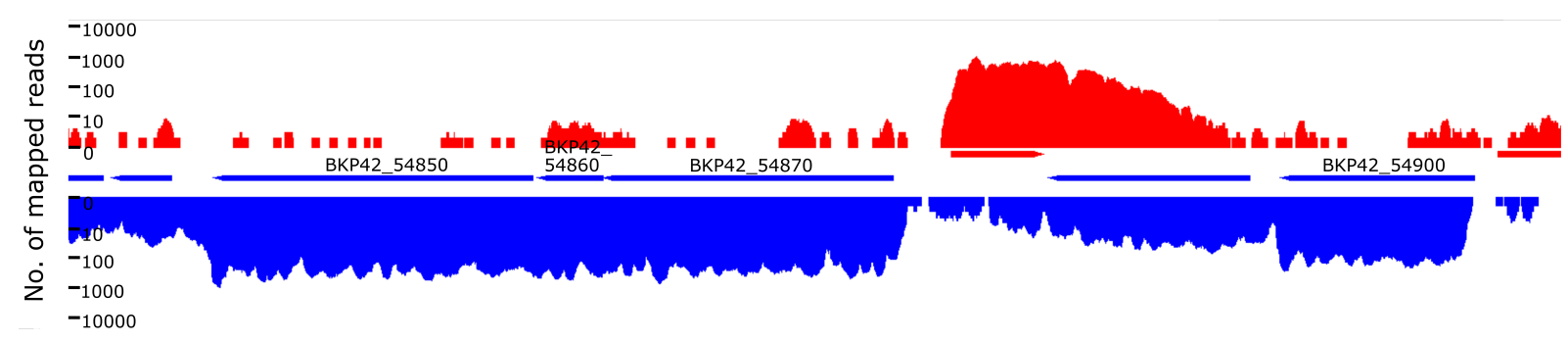

Figure 2: Expression profile of $\boldsymbol{R}$. erythropolis ACN1 under acetonitrile treatment. BKP42_54860: tubulin-like protein CetZ; BKP42_54860: hypothetical protein; BKP42_54870: tubulin/FtsZ family; GTPase domain; BKP42_54900: alipathic amidase. Arrows: position of genes; red: forward strand; blue: reverse strand.

The expression profile of the upregulated genes revealed two independently transcribed regions (Figure 2). The first contained the amidase, while the second was an operon consisting of the three remaining genes. Between both regions, only a gene encoding for an aliphatic amidase expressionregulating protein was identified on the same strand. To exclude erroneous open reading frame (ORF) prediction during previous genome annotation, NCBI ORFfinder was used to search for all possible ORFs of the respective region (Figure S1). Combination of the obtained results and the expression profile revealed the original prediction as most plausible and strengthened assumptions on identification of a novel nitrile degrading enzyme.

Metatranscriptomic screening approach. Due to the distinct results obtained for $R$. erythropolis ACN1, setup was repeated with a complex microbial community. For that purpose, a compost sample was enriched in minimal medium containing either acetonitrile (ACN) or acetic acid and ammonia (ACA) as carbon and nitrogen sources. Subsequently, fresh medium with ACN or ACA was inoculated with the precultures. Obtained growth curves showed major differences between both inoculi (Figure 3). Cultures derived from the ACN inoculum and subsequently treated with ACA reached a final optical density of 1.4 , whereas cultures containing $A C N$ reached an $\mathrm{OD}_{600}$ of 1.1. Furthermore, doubling time of the ACN-treated communities was lower than of the ACA-treated cultures. Cultures derived from the ACA inoculum behaved differently. While subsequent ACA-treatment led to fast growth and an endpoint $\mathrm{OD}_{600}$ of 1.8 , cultures treated with $\mathrm{ACN}$ showed a prolonged lag phase, slow growth, and reached only an optical density of 0.7 . 


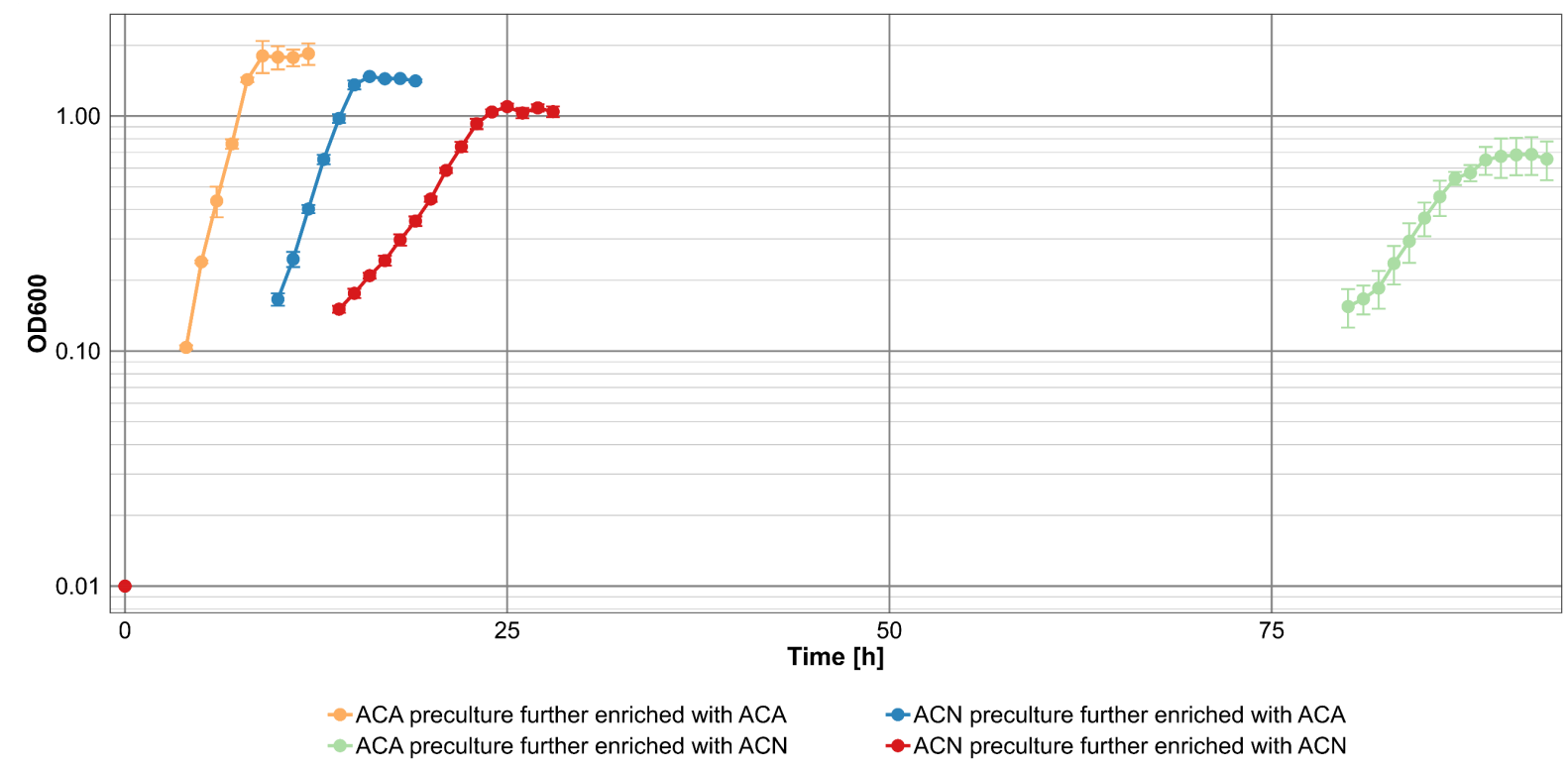

Figure 3: Growth of microbial communities under different treatments. ACA: acetic acid and ammonia; ACN: acetonitrile.

Metagenomes of both precultures as well as of all four enrichments were sequenced. After assembly, approx. 97\% of the quality-filtered reads mapped to the corresponding metagenomes (Table 3) and confirmed high quality of the assembly. In addition, a length weighted median (N50) was calculated and exhibited high values for samples derived from the ACN preculture when compared to ACAderived metagenomes.

Taxonomic analysis of the cultures revealed specific communities for each treatment (Figure 4, Data Set S2). Dominant genera in the ACN preculture were Cupriavidus (34.0\% abundance) and Pseudomonas (48.8\%). Similar relative abundances were recorded after subsequent ACA treatment ( 23.2 and $68.1 \%$, respectively), but differed in the $A C N$-treated culture ( 74.4 and $10.1 \%$, respectively). Community composition of cultures derived from the ACA inoculum differed significantly. The ACA inoculum mostly consisted of Acinetobacter (53.2\%) and Pseudomonas (42.6\%). The relative abundance of Acinetobacter increased approximately two-fold during further ACA enrichment (90.6\%), whereas Pseudomonas comprises $65.8 \%$ of the subsequently ACN-enriched community. In addition, only traces of Acinetobacter were detected in the $\mathrm{ACN}$-treated cultures. Furthermore, other organisms such as Achromobacter, Cupriavidus, and Variovorax exhibited a relative abundance over $1 \%$, although they were barely present in the preculture. 
Table 3: Metagenome data. Coverage based on bowtie2. Mapping reads: Percentage of qualityfiltered reads mapping to the assembled metagenome.

\begin{tabular}{|l|l|l|l|l|l|}
\hline Metagenome & Contigs & Size [Mb] & Coverage [x-fold] & Mapping reads [\%] & N50 [kb] \\
\hline ACA preculture & $96.3 \mathrm{k}$ & 85.7 & 46.3 & 96.9 & 1,171 \\
\hline $\begin{array}{l}\text { ACA preculture further } \\
\text { enriched with ACA }\end{array}$ & $33.1 \mathrm{k}$ & 30.4 & 166.6 & 98.5 & 1,041 \\
\hline $\begin{array}{l}\text { ACA preculture further } \\
\text { enriched with ACN }\end{array}$ & $47.6 \mathrm{k}$ & 67.6 & 80.3 & 97.3 & 3,099 \\
\hline ACN preculture & $80.5 \mathrm{k}$ & 103.8 & 51.7 & 97.2 & 2,606 \\
\hline $\begin{array}{l}\text { ACN preculture further } \\
\text { enriched with ACA }\end{array}$ & $42.6 \mathrm{k}$ & 67.4 & 61.0 & 97.2 & 5,629 \\
\hline $\begin{array}{l}\text { ACN preculture further } \\
\text { enriched with ACN }\end{array}$ & $56.8 \mathrm{k}$ & 75.5 & 144.9 & 97.3 & 4,325 \\
\hline
\end{tabular}

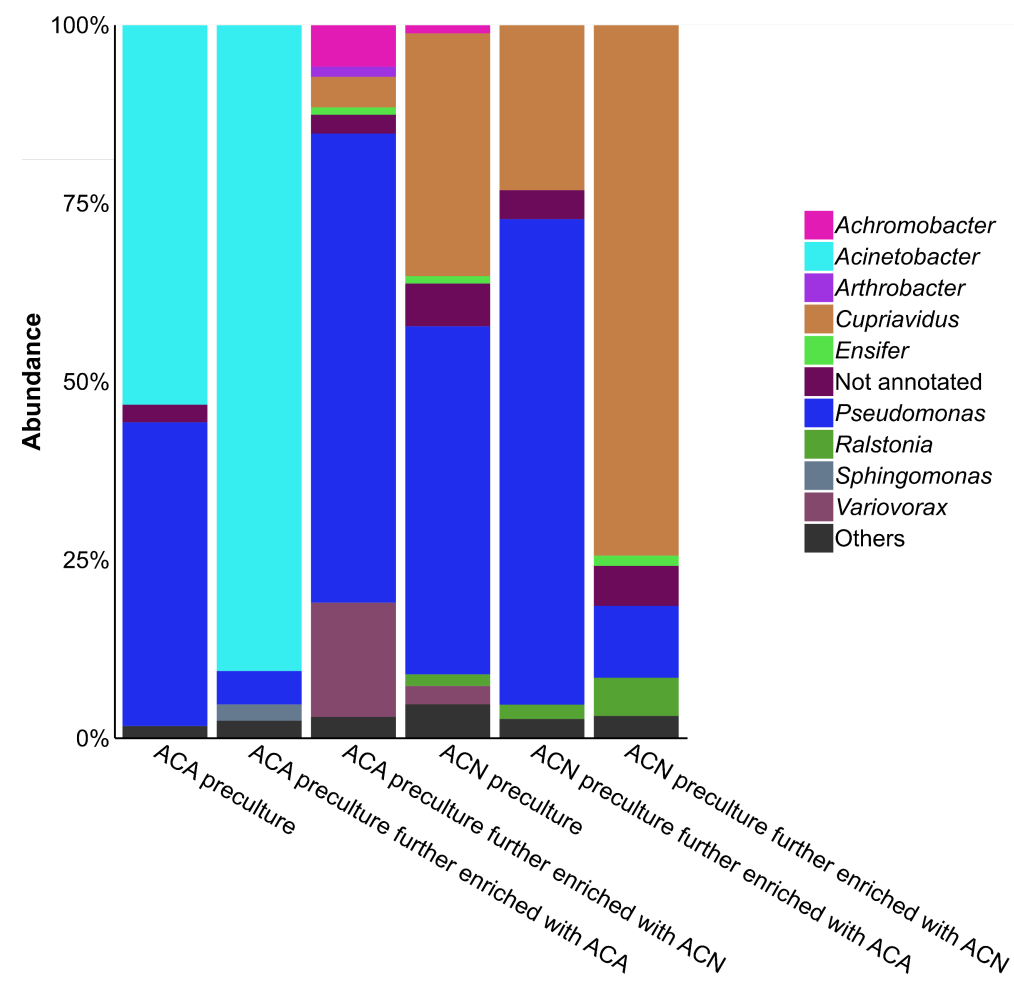

Figure 4: Composition of complex communities. Community composition based on annotation of metagenomic contigs. ACA: acetic acid and ammonia; ACN: acetonitrile. Others: Organisms with abundance $\leq 1 \%$. 
Due to the differences in community composition between cultures derived from the ACA inoculum, transcriptome normalization was not possible. Subsequent analysis was therefore performed only for ACN-derived cultures. For normalization, the previously assembled metagenomic contigs were binned and the seven largest bins belonging to Pseudomonas, Ensifer, Cupriavidus, and Flavobacterium were used for further analysis. Lowest number of quality-filtered metatranscriptomic reads was 46.9 million and on average $60.3 \%$ of the reads mapped to one of the bins.

Table 4: Number of significantly up- or downregulated genes in the largest metagenomic bins derived from ACN cultures. Differential expression was analyzed with DESeq2 with an FDR of 0.025 and $\log _{2} \mathrm{FC}$ of $\geq 2$ as cutoff.

\begin{tabular}{|l|l|l|l|}
\hline Bin & Genus & No. of upregulated genes & No. of downregulated genes \\
\hline$\# 1$ & Pseudomonas & 154 & 54 \\
\hline$\# 2$ & Ensifer & 28 & 100 \\
\hline$\# 3$ & Cupriavidus & 27 & 29 \\
\hline$\# 4$ & Pseudomonas & 186 & 65 \\
\hline$\# 5$ & Pseudomonas & 67 & 3 \\
\hline$\# 6$ & Flavobacterium & 0 & 0 \\
\hline$\# 7$ & Pseudomonas & 43 & 38 \\
\hline
\end{tabular}

After transcriptome normalization for each bin, differential analysis was performed with DESeq2 with the previously established parameters. In total, 505 up- and 289 downregulated genes were identified (Table 4, Data Set S3). Two of the Pseudomonas bins (\#1 and \#4) contained more than $66 \%$ of the upregulated genes, while no differentially expressed genes were identified for Flavobacterium. Search for genes encoding nitrile-related enzymes revealed three hits, all belonging to bin \#1. The first one is an aliphatic nitrilase ( $\left.\log _{2} \mathrm{FC} 3.2\right)$, followed by an amidase $\left(\log _{2} \mathrm{FC} 3.6\right)$ and a putative amide transporter $\left(\log _{2} \mathrm{FC} 5.4\right)$ (Data Set S3). For the other species, different groups of upregulated genes were identified. In case of Cupriavidus, five cytochrome c-related genes were upregulated, whereas another Pseudomonas (bin \#4) contained upregulated genes belonging to the nitrate/nitrite metabolism.

In addition to a metatranscriptome analysis with focus on differential gene expression of single organisms, the normalized read counts can be pooled and analyzed as a whole. Due to the accumulated counts, non-differential expression of a gene found in several of the monitored species can become significant or vice versa (19). DESeq2 analysis on the combined normalized read counts revealed 112 up- and 77 downregulated genes under $\mathrm{ACN}$ treatment. Compared with the analysis of single bins, the number of differentially expressed genes was reduced by $75.3 \%$. Of the previously identified nitrilerelated genes of bin\#1, only the putative amide transporter was still significantly upregulated in the combined dataset. In addition, three upregulated cytochrome c-related genes were observed, indicating a distinct effect of nitriles on this type of enzymes. 


\section{DISCUSSION}

During transcriptome analysis of R. erythropolis ACN1, only 23 significantly up- or downregulated genes were identified. The low number was unexpected as transcriptome studies on bacteria often identify more than 100 differentially expressed genes between two conditions $(10,20,21)$. Thus, nitriles seem to have a highly specific effect on the gene expression of nitrile-degrading organisms.

One of the upregulated genes encoded for an amidase. Together with nitrile hydratases, these enzymes are part of a bi-enzymatic pathway for nitrile degradation, but no upregulated nitrile hydratase was found. In contrast, an upregulated operon consisting of a tubulin-like protein, a hypothetical protein, and a GTPase domain of the tubulin/FtsZ family was identified. Due to proximity to the amidase, involvement in nitrile degradation appears likely. This assumption is further supported by reports on GTPase activity of nitrile hydratase activator proteins $(22,23)$. Although size of the genes does not match common nitrile hydratase subunits (24-26), it seems likely that there is similar enzymatic activity. A structurally unique nitrile hydratase of Streptomyces rimosus is published (27) and $R$. erythropolis ACN1 could contain an additional unknown structure for these enzymes. In the end, only subcloning, overexpression, and characterization of respective genes could answer questions regarding their function.

Major difference between transcriptomes of single organisms and metatranscriptomes of complex communities is the abundance of different organisms. In case of transcriptomes, all reads can be normalized on a single genetic backbone representing the sole organism in the culture. In contrast, abundance of members of a microbial community change under different treatments (28-30). An organism highly present under the first condition can be absent under the second, making normalization and comparison of metatranscriptomes difficult (19). Consequently, cultures derived from the acetic acid-treated inoculum could not be analyzed due to major changes in community composition.

Community composition of the acetonitrile-derived cultures was suitable for bioinformatic analysis, as most genera with an abundance $>1 \%$ were found in all treatments (Figure 4). After binning of the metagenomic contigs, the seven largest bins were used for subsequent normalization. Most of the bins belonged to Pseudomonas, but bins belonging to Ensifer, Cupriavidus, and Flavobacterium were also identified. Discrepancies between community composition and organisms identified by binning can be explained with the high number of different species in complex communities. For subsequent analysis, only the seven largest bins (based on bps) were considered. Although e.g. Ralstonia shows higher abundance in the community than Flavobacterium, this genus may comprise several species. Therefore, contigs of these different species would result in several different and probably smaller bins than obtained by a single Flavobacterium. Presumably as consequence of the low abundance, no 
differentially expressed genes were identified for the flavobacterial bin. Modern normalization algorithms like DESeq2 and edgeR often remove low gene read counts to suppress background noise $(15,31)$. Although deeper sequencing would solve this problem, increasing costs must be considered during experimental design.

Quality of metagenomes is often verified by two approaches $(32,33)$. The first is the percentage of quality-filtered reads mapping to the final metagenome, while the second is a size-related statistic such as N50 to compare contig lengths. All acetonitrile-derived metagenomes exhibited mapping values $>97 \%$, demonstrating the high quality of the assembly. In addition, high N50 values between 2606 and 5629 were obtained, whereas other metagenomic studies often have values below 1800 $(32,34,35)$. This discrepancy arises presumably from the low diversity of our microbial communities, as high coverage of a metagenome assists during the assembly of long contigs and therefore leads to high N50 values. Additional sequencing could probably further increase their quality, but based on the mapping and N50 values, the here obtained metagenomes were suitable as backbone for metatranscriptome normalization.

A first analysis of the binned metatranscriptomes demonstrated the potential of the novel screening approach. The upregulated nitrilase and amidase identified for Pseudomonas (bin \#1) indicate nitrile degradation by at least one of the possible pathways. It can be assumed that the putative amide transporter leads to an export of amides into the medium and therefore to cross-feeding of other community members. This assumption is further supported by another Pseudomonas (bin \#4), as its upregulated nitrate/nitrite metabolism suggests other methods than nitrile degradation to be involved in nitrogen acquisition. Thus, separate transcriptome analysis for each bin can give insights into metabolic networks of microbial communities and probably help to optimize them for bioremediation purposes.

Additional analysis of the combined metatranscriptomic read counts highlighted differences between the two analytical methods. While the upregulated nitrilase and amidase of Pseudomonas (bin \#1) lost statistical significance, the putative amide transporter was still identified. Probably, similar genes were expressed in other members of the community but removed during bioinformatical analysis due to low read counts. In contrast, after combination of all read counts the obtained signal was strong enough to be detected. Thus, no insights into specific responses of single community members can be obtained after pooling of read counts, but larger pattern of differential expression can be observed. In conclusion, a combination of both approaches is recommended to harness the full potential of metatranscriptomics (19). 


\section{CONCLUSION AND OUTLOOK}

The here presented screening approach demonstrates the potential of differential transcriptomics. During analysis of the R. erythropolis ACN1 transcriptomes, only four significantly upregulated genes were identified under nitrile treatment. The experimental setup seems to result in highly specific expression pattern and can therefore assist in the identification of novel biocatalysts. One of the upregulated genes belonged to an amidase, fitting our expectations, whereas the remaining upregulated genes did not belong to known nitrile-degrading enzymes. Involvement in nitrile degradation can be assumed due to the strong effect of nitriles on their expression, but further research must be done for functional assignment.

First analysis of the metatranscriptomes highlighted differences between transcriptomes of single community members and the combined read counts. Although a nitrilase most likely involved in acetonitrile degradation was identified, additional analyses must be done to uncover all enzymes involved in nitrile assimilation. Upregulated hypothetical proteins are interesting candidates for novel nitrile-degrading enzymes and especially hits found for single bins as well as the combined metatranscriptome are of major interest. In addition, binning and metatranscriptome normalization could be improved by transition to a metagenomic backbone consisting of the pooled reads of all acetonitrile-derived.

Major limitation of the presented screening approach is the enrichment of microbial communities and therefore a loss of diversity. To minimize this effect, complex communities could be grown in bioreactors. Due to better aeration and constant nutrient supply, a higher diversity should be maintained. In addition, short-term incubation of an environmental sample with a nitrile or the respective carboxylic acid and ammonia could be applied. This approach would reduce loss of diversity to a minimum but requires a high sequencing depth to gather sufficient information for subsequent analysis.

In conclusion, the here presented method provides a first insight into effects of acetonitrile on metabolism of a complex community. Additional analysis of the metatranscriptomes and especially of upregulated putative genes and the corresponding proteins would lead to identification of additional nitrile-degrading enzymes and effects. In future, this approach might probably be routinely used for identification of novel biocatalysts as it does not depend on suitable screening strategies for different substrates and can therefore be easily adapted. 


\section{MATERIAL AND METHODS}

Media. M9 medium (36) was prepared containing $6 \mathrm{~g} \mathrm{Na}_{2} \mathrm{HPO}_{4}, 3 \mathrm{~g} \mathrm{KH} \mathrm{PO}_{4}, 1 \mathrm{~g} \mathrm{NH}_{4} \mathrm{Cl}$, and $0.5 \mathrm{~g} \mathrm{NaCl}$ per liter. After autoclaving, $10 \mathrm{ml}$ ATCC trace mineral supplement, $10 \mathrm{ml}$ ATCC vitamin supplement (both LGC Standards, Teddington, UK), $10 \mathrm{ml}$ glucose solution (20\%), $1 \mathrm{ml} \mathrm{MgSO} \cdot \mathrm{M} \mathrm{H}_{2} \mathrm{O}$ solution $(1 \mathrm{M}), 1 \mathrm{ml} \mathrm{CaCl}$ solution $(14.7 \mathrm{~g} / \mathrm{l})$, and $1 \mathrm{ml}$ thiamine- $\mathrm{HCl}$ solution $(1 \mathrm{mg} / \mathrm{ml})$ were added. For differential transcriptomics, either $25 \mathrm{mM}$ acetonitrile or $25 \mathrm{mM}$ acetic acid and ammonia were used as sole carbon and nitrogen sources.

Enrichment of an environmental sample. $100 \mathrm{~g}$ compost $(\mathrm{pH} \mathrm{7.5)}$ of the Experimental Botanical

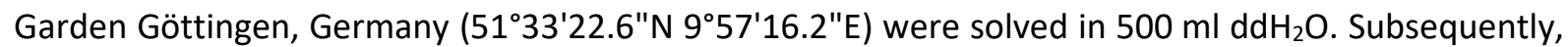
the solution was filtered with a $2.7 \mu \mathrm{m}$ GF/D glass fiber filter (Whatman, Little Chalfont, UK) and $5 \mathrm{ml}$ of it were used to inoculate $45 \mathrm{ml} \mathrm{M9}$ with acetonitrile or acetic acid and ammonia in $300 \mathrm{ml}$ baffled flasks. Incubation was performed at $25^{\circ} \mathrm{C}$ and $180 \mathrm{rpm}$ (Innova 44 incubator). Two prevent starvation, cultures were transferred to fresh medium after reaching approx. an $\mathrm{OD}_{600}$ of 1.0. New cultures were inoculated with an $\mathrm{OD}_{600}$ of 0.1 and further incubated. In total, four rounds of incubation were performed.

Growth conditions. For single transcriptome studies, a preculture of $R$. erythropolis ACN1 (14) was grown over night in $5 \mathrm{ml} \mathrm{M9} \mathrm{medium.} \mathrm{For} \mathrm{metatranscriptome} \mathrm{studies,} \mathrm{a} \mathrm{previously} \mathrm{enriched}$ environmental soil sample was used (see before). Precultures were washed twice with M9 containing no carbon or nitrogen sources. The samples were used to inoculate $50 \mathrm{ml} \mathrm{M9}$ with acetonitrile, or acetic acid and ammonia in $300 \mathrm{ml}$ baffled flasks to an $\mathrm{OD}_{600}$ of 0.01 . Incubation was performed at $25^{\circ} \mathrm{C}$ and $180 \mathrm{rpm}$ using an Innova 44 incubator (New Brunswick Scientific, Nürtingen, Germany). Growth of cultures was measured regularly and samples for transcriptome sequencing were harvested during logarithmic growth phase at an $\mathrm{OD}_{600}$ of approx. 1.0 (R. erythropolis $\mathrm{ACN} 1$ ) or 0.4 (metagenomes). For harvesting, samples were centrifuged for $1 \mathrm{~min}$ at $10000 \times g$ and supernatant was discarded. Afterwards, samples were directly frozen in liquid $\mathrm{N}_{2}$ and subsequently stored at $-80^{\circ} \mathrm{C}$. All conditions were prepared as triplicates.

DNA isolation. DNA of the enrichment cultures was isolated using the Lucigen MasterPure Complete DNA and RNA Purifcation kit (Lucigen, Middleton, WI, USA) according to the manufacturer's protocol. DNA of the metatranscriptome cultures was isolated with the same kit but with a modified protocol to resemble the cell disruption of the RNA isolation procedure. Pellets were dissolved in $300 \mu \mathrm{l} \mathrm{TC} \mathrm{buffer}$ containing $2 \mu \mathrm{l}$ proteinase $\mathrm{K}$. Subsequently, samples were transferred to a cell mill (MikroDismembrator U, B. Braun, Melsungen, Germany) containing liquid $N_{2}$ and disrupted at 1,600 rpm for $3 \mathrm{~min}$. The frozen cell powder was dissolved in $1.5 \mathrm{ml} \mathrm{TC}$ buffer containing $6 \mu$ proteinase $\mathrm{K}$ and DNA 
was isolated as recommended by Lucigen. Final DNA concentration was measured using a Qubit fluorometer with dsDNA HS assay kit as recommended by the manufacturer (Invitrogen, Carlsbad, CA, USA).

Metagenome sequencing and analysis. DNA of triplicate cultures was pooled in equal amounts. Paired-end sequencing $(2 \times 300 \mathrm{bp})$ was performed on an Illumina MiSeq using the Nextera XT DNA library prep kit and v3 chemistry as described by the manufacturer (Illumina, San Diego, CA, USA). Quality trimming of the paired-end reads was done with Trimmomatic version 0.38 (37) and validated with FastQC version 0.11 .7 (38). For assembly, SPAdes version 3.12 (39) was used. All contigs $>200$ bp were annotated with Prokka 1.13.3 (40). Coverage was calculated using Bowtie 2 version 2.3.4.2 (41) and all contigs were checked with BLASTn (42) against the NCBI non-redundant database for taxonomical assignment.

RNA isolation. Cell pellets were solved in $400 \mu \mathrm{l}$ RLT buffer from the RNeasy Mini Kit (Qiagen, Hilden, Germany) containing $4 \mu \mathrm{l} \beta$-mercaptoethanol. Subsequently, cell disruption was performed in a cell mill (Mikro-Dismembrator $\mathrm{U}, \mathrm{B}$. Braun) containing liquid $\mathrm{N}_{2}$ for $3 \mathrm{~min}$ at 1,600 rpm. Cell powder was dissolved in $400 \mu \mathrm{l} \mathrm{RLT}$ buffer containing $4 \mu \mathrm{l} \beta$-mercaptoethanol and $1.2 \mathrm{ml}$ ethanol. Subsequently, the RNA isolation was done according to the manufacturer's protocol, but with RWT instead of RW1 buffer. Final RNA concentration was measured with a Qubit using the RNA BR assay kit (Invitrogen), whereas RNA quality was controlled with a 2100 Bioanalyzer using the RNA 6000 Nano kit (Agilent Technologies, Waldbronn, Germany).

Transcriptome sequencing. Remaining DNA after RNA isolation was degraded with Turbo DNase (Thermo Fisher Scientific) and subsequently purified with the RNeasy MinElute Cleanup kit using $350 \mu \mathrm{l}$ RLT buffer with $3.5 \mu \mathrm{l} \beta$-mercaptoethanol and $675 \mu \mathrm{l}$ ethanol. After total DNA removal, rRNA depletion was done with the Ribo-Zero rRNA removal kit for bacteria (Illumina) as recommended by the supplier. Non-directed sequencing libraries were built with the NEBNext Ultra II RNA Library Prep kit (New England BioLabs) for Illumina sequencers according to the manufacturer's protocol. Final validation of the cDNA library was done using a Qubit with the dsDNA HS assay kit (Invitrogen) and with a 2100 Bioanalyzer using the DNA High Sensitivity kit (Agilent Technologies). Single-end sequencing was done on an Illumina HiSeq 4000 (Illumina) with $1 \times 50$ bp for R. erythropolis ACN1 transcriptomes and $1 \times 100$ bp for metatranscriptomes.

(Meta-)Transcriptome analysis. Quality trimming of the single-end reads was done with Trimmomatic version 0.38 (37) and validated with FastQC version 0.11 .7 (38). Normalization of the R. erythropolis ACN1 transcriptomes was done with R version 3.5.1 (43) and DESeq2 package version 1.20.0 (15). For normalization of the metatranscriptomes, reads were mapped against the preculture metagenome 
with bowtie2 (version 2.3.4.3) (41). The mappings were used for the coverage profiles and for the number of reads assigned to a gene for the differential expression analysis. In order to compare proteins from different organisms, the protein features were unified via the KEGG ORTHOLOGY database (KO, date: 2018-10-15) (44). All proteins with a KO identifier were stored in a DIAMOND (v0.9.22.123) database (45). Proteins identified for the preculture metagenome contigs via Prokka were KO annotated using the DIAMOND database. For annotation, a target coverage of at least $90 \%$ was required and it was possible for a protein to have multiple annotations. To obtain bins of organisms, MetaBAT (version 0.32.4) (46) was used with default parameters, but --minSamples with 6 instead of 10 (Minimum number of sample sizes for considering correlation based recruiting). For the $\mathrm{DE}$ analysis, the 7 largest bins (bp wise) were selected, ranging from 6.8 to $2.6 \mathrm{Mbp}$. The features for the $\mathrm{DE}$-analysis were obtained by counting the number of transcriptome reads overlapping with regions of the annotated KOs for each contig and summing up the KOs from contigs with the same bin id. The reads for each bin were normalized separately with DESeq2 (15) and then merged for the metatranscriptom analysis (with no further normalization). DESeq2 results with an FDR cutoff of 0.025 were subsequently filtered by just considering genes with a logarithmic fold-change of $\geq 2$ as differentially expressed.

For graphical analysis, trimmed reads of the R. erythropolis ACN1 transcriptomes obtained after acetonitrile treatment were pooled. Number of reads was reduced by random subsampling by factor 10 and remaining reads were mapped with bowtie2 against the $R$. erythropolis ACN1 genome. Visualization of the results was done with TraV (47).

For additional ORF prediction, NCBI ORFfinder was used with genetic code 11. ATG and alternative initiation codons were used and nested ORFs ignored. ORFs smaller than 300 bp were not displayed.

Accession numbers. Biosample accession numbers for raw data of (meta)genome and (meta)transcriptome sequencing as well as assembled (meta)genomes are the following: R. erythropolis ACN1, SAMN05788061; acetonitrile preculture, SAMN10107935; acetonitrile preculture further enriched with acetonitrile, SAMN10112108; acetonitrile preculture further enriched with acetic acid, SAMN10112109; acetic acid preculture, SAMN10112120; acetic acid preculture further enriched with acetonitrile, SAMN10112123; acetic acid preculture further enriched with acetic acid, SAMN10112148.

\section{FUNDING INFORMATION}

The work of R.E. was supported by the "Fonds der Chemischen Industrie im Verband der Chemischen Industrie e.V.". The funders had no role in study design, data collection, and interpretation, or the decision to submit the work for publication. 


\section{REFERENCES}

1. Conn EE. 1969. Cyanogenic glycosides. J Agric Food Chem 17:519-526.

2. Kurenkov VF. 1997. Acrylamide polymers., p. 61-72. In Cheremisinoff, NP (ed.), Handbook of engineering polymeric materials, 1st ed. Marcel Dekker, New York, NY, USA.

3. Fleming FF, Yao L, Ravikumar PC, Funk L, Shook BC. 2010. Nitrile-containing pharmaceuticals: Efficacious roles of the nitrile pharmacophore. J Med Chem 53:7902-7917.

4. Shaw NM, Robins KT, Kiener A. 2003. Lonza: 20 years of biotransformations. Adv Synth Catal 345:425-435.

5. Panova A, Mersinger LJ, Liu Q, Foo T, Roe DC, Spillan WL, Sigmund AE, Ben-Bassat A, Wagner LW, O'Keefe DP, Wu S, Petrillo KL, Payne MS, Breske ST, Gallagher FG, DiCosimo R. 2007. Chemoenzymatic synthesis of glycolic acid. Adv Synth Catal 349:1462-1474.

6. Gong J-S, Lu Z-M, Li H, Shi J-S, Zhou Z-M, Xu Z-H. 2012. Nitrilases in nitrile biocatalysis: Recent progress and forthcoming research. Microb Cell Fact 11:142.

7. Uchiyama T, Miyazaki K. 2009. Functional metagenomics for enzyme discovery: Challenges to efficient screening. Curr Opin Biotechnol 20:616-622.

8. Simon C, Daniel R. 2011. Metagenomic analyses: Past and future trends. Appl Environ Microbiol 77:1153-1161.

9. Hurtado Páez UA, García Romero IA, Restrepo Restrepo S, Aristizábal Gutiérrez FA, Montoya Castaño D. 2015. Assembly and analysis of differential transcriptome responses of Hevea brasiliensis on interaction with Microcyclus ulei. PLoS One 10:e0134837.

10. Yang J-W, Zheng D-J, Cui B-D, Yang M, Chen Y-Z. 2016. RNA-seq transcriptome analysis of a Pseudomonas strain with diversified catalytic properties growth under different culture medium. Microbiologyopen 5:626-636.

11. Zhang J, Chen L, Fu C, Wang L, Liu H, Cheng Y, Li S, Deng Q, Wang S, Zhu J, Liang Y, Li P, Zheng A. 2017. Comparative transcriptome analyses of gene expression changes triggered by Rhizoctonia solani AG1 IA infection in resistant and susceptible rice varieties. Front Plant Sci 8:1422.

12. Dillies MA, Rau A, Aubert J, Hennequet-Antier C, Jeanmougin M, Servant N, Keime C, Marot NS, Castel D, Estelle J, Guernec G, Jagla B, Jouneau L, Laloë D, Le Gall C, Schaëffer B, Le Crom S, Guedj M, Jaffrézic F. 2013. A comprehensive evaluation of normalization methods for Illumina high-throughput RNA sequencing data analysis. Brief Bioinform 14:671-683.

13. Lin Y, Golovnina K, Chen Z-X, Lee HN, Negron YLS, Sultana H, Oliver B, Harbison ST. 2016. Comparison of normalization and differential expression analyses using RNA-Seq data from 726 individual Drosophila melanogaster. BMC Genomics 17:28.

14. Egelkamp R, Schneider D, Hertel R, Daniel R. 2017. Nitrile-degrading bacteria isolated from compost. Front Environ Sci 5:56.

15. Love MI, Huber W, Anders S. 2014. Moderated estimation of fold change and dispersion for RNA-seq data with DESeq2. Genome Biol 15:550. 
16. Bedre R, Rajasekaran K, Mangu VR, Timm LES, Bhatnagar D, Baisakh N. 2015. Genome-wide transcriptome analysis of cotton (Gossypium hirsutum L.) identifies candidate gene signatures in response to aflatoxin producing fungus Aspergillus flavus. PLoS One 10:e0138025.

17. Gupta S, Shi X, Lindquist IE, Devitt N, Mudge J, Rashotte AM. 2013. Transcriptome profiling of cytokinin and auxin regulation in tomato root. J Exp Bot 64:695-704.

18. Newman MS, Nguyen T, Watson MJ, Hull RW, Yu H-G. 2017. Transcriptome profiling reveals novel BMI- and sex-specific gene expression signatures for human cardiac hypertrophy. Physiol Genomics 49:355-367.

19. Klingenberg $\mathrm{H}$, Meinicke P. 2017. How to normalize metatranscriptomic count data for differential expression analysis. PeerJ 5:e3859.

20. Anfelt J, Hallström B, Nielsen J, Uhlén M, Hudson EP. 2013. Using transcriptomics to improve butanol tolerance of Synechocystis sp. strain PCC 6803. Appl Environ Microbiol 79:7419-7427.

21. Schwartz T, Armant O, Bretschneider N, Hahn A, Kirchen S, Seifert M, Dötsch A. 2015. Whole genome and transcriptome analyses of environmental antibiotic sensitive and multi-resistant Pseudomonas aeruginosa isolates exposed to waste water. and tap water. Microb Biotechnol 8:116-130.

22. Duca D, Rose DR, Glick BR. 2014. Characterization of a nitrilase and a nitrile hydratase from Pseudomonas sp. strain UW4 that converts indole-3-acetonitrile to indole-3-acetic acid. Appl Environ Microbiol 80:4640-4649.

23. Gumataotao N, Lankathilaka KPW, Bennett B, Holz RC. 2017. The iron-type nitrile hydratase activator protein is a GTPase. Biochem J 474:247-258.

24. Prasad S, Bhalla TC. 2010. Nitrile hydratases (NHases): At the interface of academia and industry. Biotechnol Adv 28:725-741.

25. Huang W, Jia J, Cummings J, Nelson M, Schneider G, Lindqvist Y. 1997. Crystal structure of nitrile hydratase reveals a novel iron centre in a novel fold. Structure 5:691-699.

26. Nakasako M, Odaka M, Yohda M, Dohmae N, Takio K, Kamiya N, Endo I. 1999. Tertiary and quaternary structures of photoreactive Fe-type nitrile hydratase from Rhodococcus sp. N-771: Roles of hydration water molecules in stabilizing the structures and the structural origin of the substrate specificity of the enzyme. Biochemistry 38:9887-9898.

27. Nelp MT, Astashkin A V, Breci LA, McCarty RM, Bandarian V. 2014. The alpha subunit of nitrile hydratase is sufficient for catalytic activity and post-translational modification. Biochemistry 53:3990-3994.

28. Kim HB, Borewicz K, White BA, Singer RS, Sreevatsan S, Tu ZJ, Isaacson RE. 2012. Microbial shifts in the swine distal gut in response to the treatment with antimicrobial growth promoter, tylosin. Proc Natl Acad Sci 109:15485-15490.

29. Svensson K, Paruch L, Gaby JC, Linjordet R. 2018. Feeding frequency influences process performance and microbial community composition in anaerobic digesters treating steam exploded food waste. Bioresour Technol 269:276-284. 
30. Calicioglu O, Shreve MJ, Richard TL, Brennan RA. 2018. Effect of pH and temperature on microbial community structure and carboxylic acid yield during the acidogenic digestion of duckweed. Biotechnol Biofuels 11:275.

31. Robinson MD, McCarthy DJ, Smyth GK. 2010. edgeR: A Bioconductor package for differential expression analysis of digital gene expression data. Bioinformatics 26:139-140.

32. Papudeshi B, Haggerty JM, Doane M, Morris MM, Walsh K, Beattie DT, Pande D, Zaeri P, Silva GGZ, Thompson F, Edwards RA, Dinsdale EA. 2017. Optimizing and evaluating the reconstruction of metagenome-assembled microbial genomes. BMC Genomics 18:915.

33. Forouzan E, Shariati P, Mousavi Maleki MS, Karkhane AA, Yakhchali B. 2018. Practical evaluation of 11 de novo assemblers in metagenome assembly. J Microbiol Methods 151:99-105.

34. Mason OU, Hazen TC, Borglin S, Chain PSG, Dubinsky EA, Fortney JL, Han J, Holman HYN, Hultman J, Lamendella R, MacKelprang R, Malfatti S, Tom LM, Tringe SG, Woyke T, Zhou J, Rubin EM, Jansson JK. 2012. Metagenome, metatranscriptome and single-cell sequencing reveal microbial response to Deepwater Horizon oil spill. ISME J 6:1715-1727.

35. Magasin JD, Gerloff DL. 2015. Pooled assembly of marine metagenomic datasets: Enriching annotation through chimerism. Bioinformatics 31:311-317.

36. Atlas RM. 2010. Handbook of microbiological media., 4th ed. CRC Press, Boca Raton, FL, USA.

37. Bolger AM, Lohse M, Usadel B. 2014. Trimmomatic: a flexible trimmer for Illumina sequence data. Bioinformatics 30:2114-2120.

38. Andrews S. 2010. FastQC: a quality control tool for high throughput sequence data.

39. Bankevich A, Nurk S, Antipov D, Gurevich AA, Dvorkin M, Kulikov AS, Lesin VM, Nikolenko SI, Pham S, Prjibelski AD, Pyshkin A V, Sirotkin A V, Vyahhi N, Tesler G, Alekseyev MA, Pevzner PA. 2012. SPAdes: A new genome assembly algorithm and its applications to single-cell sequencing. J Comput Biol 19:455-477.

40. Seemann T. 2014. Prokka: Rapid prokaryotic genome annotation. Bioinformatics 30:20682069.

41. Langmead B, Salzberg SL. 2012. Fast gapped-read alignment with Bowtie 2. Nat Methods 9:357359.

42. Altschul SF, Gish W, Miller W, Myers EW, Lipman DJ. 1990. Basic local alignment search tool. J Mol Biol 215:403-410.

43. R Core Team. 2018. R: A language and environment for statistical computing. R Foundation for Statistical Computing, Vienna, Austria.

44. Kanehisa M, Furumichi M, Tanabe M, Sato Y, Morishima K. 2017. KEGG: New perspectives on genomes, pathways, diseases and drugs. Nucleic Acids Res 45:D353-D361.

45. Buchfink B, Xie C, Huson DH. 2014. Fast and sensitive protein alignment using DIAMOND. Nat Methods 12:59-60.

46. Kang DD, Froula J, Egan R, Wang Z. 2015. MetaBAT, an efficient tool for accurately reconstructing single genomes from complex microbial communities. PeerJ 3:e1165. 
47. Dietrich S, Wiegand S, Liesegang H. 2014. TraV: a genome context sensitive transcriptome browser. PLoS One 9:e93677. 
Chapter IV

General discussion 


\section{General discussion}

Nitriles are widespread in nature and important compounds in industry. In natural systems, nitrile synthesis should equal nitrile degradation to avoid accumulation of these toxic molecules, but industrial leakage or improper disposal lead to contamination of the environment. For reduction of nitrile pollution the use of nitrile-converting enzymes and organisms as biocatalysts or in bioremediation is a promising approach. Therefore, identification of novel biocatalysts is important to expand the toolbox for green chemistry. This task is hindered by limitations of current function- or sequence-based screening approaches like (problematic) expression in heterologous hosts or inability to identify truly novel enzymes $(128,133)$.

In this study, a novel approach for identification of nitrile-degrading enzymes based on reverse omics techniques was developed. This approach is based on differential analysis of (meta)transcriptomic datasets without necessity of designing a suitable screening system. For this purpose, bacterial cultures are grown either with a nitrile or the corresponding products of the nitrilase reaction, carboxylic acids and ammonia. Subsequently, (meta)transcriptomes are sequenced and analyzed. Genes upregulated in presence of the nitrile could encode e.g. nitrile-degrading enzymes, transporters, or resistance mechanisms. The thereby gathered data could finally lead to the identification of novel biocatalysts. As nitriles are often toxic, understanding of their influence on microbial growth was necessary for establishing the novel screening approach. Therefore, the effect of different nitriles and their corresponding carboxylic acids on microbial growth was determined for single strains and complex communities (Chapter 3.1). One of the nitriles showed growth-supporting effects, indicating its utilization as additional nutrient source. Isolation of respective organisms led to the identification of eight novel acetonitrile-degrading bacterial strains (Chapter 3.2). In addition, a novel high-throughput nitrilase assay was developed. Subsequent screening of 70 putative nitrilases led to the identification and characterization of a new arylacetonitrilase (Chapter 3.3). Finally, a novel (meta)transcriptomic screening approach was developed. One of the previously isolated nitrile-degrading organisms was used to establish screening conditions and the subsequent bioinformatic workflow. Afterwards, a complex microbial community was identically treated and used for the first nitrilase screening approach based on differential metatranscriptomics (Chapter 3.4).

\subsection{Nitriles and microbial life}

Microbial communities are key players in most nutrient cycles and important for ecosystem functioning (137-143). Consequently, their natural diversity and composition (144-147) as well as influence of factors like pH (148), soil moisture (149), humidity (150), temperature (151), or salinity 
(152) has been studied in detail. In addition to these environmental properties, man-made impact due to substances such as fertilizers (153), antibiotics (154), herbicides (155), or heavy metals (156) has been determined. In contrast, influence of often toxic nitriles on microbial communities is almost unknown. Besides unintended release of industrially used nitriles, they are also actively released in form of herbicides with negative impact on microbial biomass (157). Furthermore, many important crops contain cyanogenic glycosides (Table 1). For example, approximately 250 million tons of cassava with up to $1.5 \mathrm{~g}$ cyanide equivalent per kilogram are produced per year $(158,159)$. Disposed scraps of all these crops can rot in the environment and lead to increased nitrile concentrations. In addition, carboxylic acids released by nitrile degradation may also influence local biota $(160,161)$.

Table 1: Agriculturally important plants containing cyanogenic glycosides.

\begin{tabular}{|l|l|l|}
\hline Occurrence & Common name(s) & References \\
\hline Anacardium occidentale & Cashew & $(162)$ \\
\hline Bambusa spp. & Bamboo & $(163)$ \\
\hline Cicer arietinum & Chickpea & $(164)$ \\
\hline Eucalyptus spp. & Eucalyptus & $(165)$ \\
\hline Linium spp. & Flax & $(166)$ \\
\hline Malus spp. & Apple & $(167)$ \\
\hline Manihot esculenta & Cassava & $(168)$ \\
\hline Pangium edule & Keluak & $(169)$ \\
\hline Passiflora spp. & Passion fruit & $(170)$ \\
\hline Phaseolus lunatus & Lima bean & $(171)$ \\
\hline Prunus spp. & Almond, apricot, cherry, peach, plum & $(162,172,173)$ \\
\hline Sambucus spp. & Elderberry & $(173,174)$ \\
\hline Simmondsia chinensis & Jojoba & $(175)$ \\
\hline Sorghum spp. & Sorghum & $(176)$ \\
\hline Trifolium repens & White clover & $(177)$ \\
\hline Vicia sativa & Common vetch & $(173)$ \\
\hline
\end{tabular}

For a first insight into possible effects of nitriles and their corresponding carboxylic acids, influence on bacterial growth was studied (Chapter 3.1). Previous toxicity experiments on mammals have shown diverging effects of structurally different nitriles (63). Consequently, structurally diverse substrates were chosen in this study. In total, nine different mononitriles, dinitriles, cyanohydrin derivatives, arylacetonitriles, aromatic nitriles, cyclohexane nitriles (Figure 1), and their corresponding carboxylic acids were selected. 


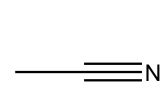

Acetonitrile

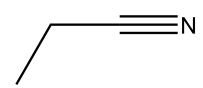

Pyruvonitrile

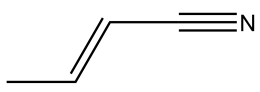

Crotononitrile

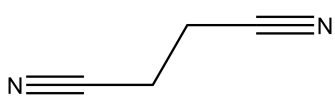

Succinonitrile

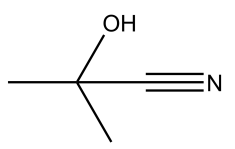

Acetone cyanohydrin

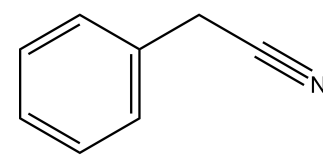

Phenylacetonitrile

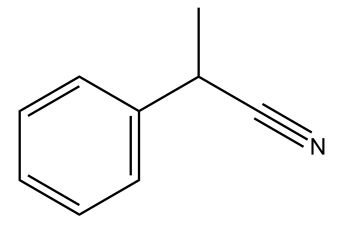

2-Phenylpropionitrile

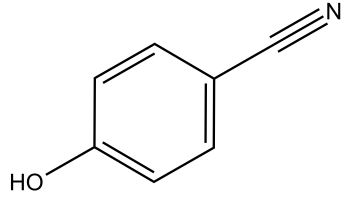

4-Hydroxybenzonitrile

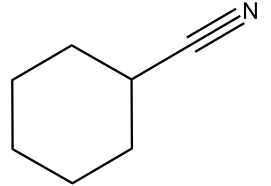

Cyclohexanecarbonitrile

Figure 1: Nitriles used as substrates for toxicity determination.

After determination of nitrile toxicity on Agrobacterium tumefaciens, Bacillus subtilis, Corynebacterium glutamicum, and Escherichia coli using solid media, effect of the nitriles and carboxylic acids on a complex microbial community was analyzed. Compost was chosen as starting material to increase chance for viable communities due to its higher diversity compared to aquatic environments (178). Common methods for extraction of similar communities use serial dilutions (179) or sonication and subsequent low-speed centrifugation $(180,181)$. The first method leads to dilution of microorganisms and therefore loss of biomass, whereas the second method can severely damage the cells and reduce number of viable organisms (181). Consequently, a different approach was tested. For that purpose, compost was solved in $\mathrm{H}_{2} \mathrm{O}$ and filtered with a $2.7 \mu \mathrm{m}$ glass fiber filter to remove matrix particles. Pore size of the filter was chosen to allow flowthrough of presumably the largest bacterium found in German soil, Bacillus megaterium (182). 16S rRNA gene analysis of the unfiltered and filtered soil sample revealed very similar community compositions with more than 500 different organisms at genus level and identical Simpson indices of diversity (SID) values. Thus, it is indicated that the here established method is suitable for removal of compost or soil particles in future studies of similar communities.

During toxicity determination, two important results were retrieved. First is the high toxicity of 4-hydroxybenzonitrile, leading to suppressed growth and reduced diversity. Structurally similar herbicides like bromoxynil and ioxynil show similar impact on microbes $(157,183)$. Thus, it can be assumed that the use of 4-hydroxybenzonitrile-derived herbicides has severe influence on soil microbiota. More than 1,300 tons of bromoxynil have been used in the US in 2016 (184) and most likely the use of ioxynil is even more widespread as it is also available for private households. Transition to less toxic herbicides should be considered to increase quality of soil microbiota and therefore crop yield (185).

The second important result is the lethal effect of acetone cyanohydrin. This cyanohydrin is the degradation product of linamarin, a cyanogenic glycoside found in cassava, lima beans, and flax (186-188). Due to large-scale cultivation of these crops, release of acetone cyanohydrin by rotting 
scraps could be a source for environmental contamination and hazardous for local microbiota. Nevertheless, further studies must be performed for estimation of the impact of cyanogenic crops on the environment.

In addition to specific levels of toxicity, a higher nitrile susceptibility of Gram-negatives compared to Gram-positives was recorded. Research on tolerance or resistance of both bacterial groups to antibiotics, hydrocarbons, cold plasma, or special nanoparticles has been performed before (189-194), but this is the first time that nitrile-susceptibility has been studied. Due to the small number of tested compounds and organisms, these findings can just serve as tendency. Further studies including other nitriles and several different communities are necessary.

\subsection{Acetonitrile-degrading bacteria}

Acetonitrile is an industrially important nitrile often employed as solvent. It is used during the production of butadiene (195), a key solvent in pharmacy (196), and part of battery electrolytes (197). In addition, it is important for the synthesis of oligonucleotides (198) and often used for liquid chromatography (199). Nevertheless, it exhibits higher toxicity than other solvents such as methanol or acetone and is regarded as problematic waste $(199,200)$. Conventional treatment of acetonitrile waste can be done by addition of large quantities of sodium hydroxide (201), with supercritical water and $\mathrm{H}_{2} \mathrm{O}_{2}$ (202), by photolysis (203), or via combustion in a hazardous waste plant (201). As most of these processes need unfavorable chemicals or conditions, interest in bioremediation of acetonitrilecontaining waste is increasing (204-206). During analysis of nitrile toxicity on microbial communities (Chapter 3.1), cultures containing acetonitrile exhibited higher optical density than the control. As nitrile-metabolization and therefore presence of nitrile-degrading enzymes could be assumed, isolation of respective organisms was performed (Chapter 3.2).

Eight unique strains belonging to Flavobacterium, Pseudomonas, Rhodococcus, and Variovorax were isolated. Four of the eight isolates belonged to Pseudomonas, demonstrating its well-known acetonitrile-degrading abilities (207-209). In addition, one Rhodococcus isolate was identified. This genus is probably the most prominent bacterial genus for nitrile bioremediation and biocatalysis. It can degrade a large number of compounds including acetonitrile (210-212), also reflecting by the increasing number of related patents (213). Despite these common nitrile degraders, one isolate of Variovorax and two isolates of Flavobacterium were obtained. Nitrile-degrading ability of Variovorax was described before $(214,215)$, but only weak hydrolysis of nicotinonitrile by Flavobacterium has previously been observed (216). Due to missing genomes or enzymatic studies, the pathway involved in nicotinonitrile degradation remains unknown. Although no nitrilases or nitrile hydratases could be annotated in the genomes of the flavobacterial isolates, these results indicate that nitrile degradation 
is even more widespread than previously known. Further studies are required to unravel the underlying mechanism and increase the number of nitrile-degrading biocatalysts.

\subsection{High-throughput nitrilase assays}

In addition to identification of nitrile-degrading bacteria (Chapter 3.2), characterization of putative nitrile-degrading enzymes is of increasing importance. To analyze the growing amount of putative nitrilases and nitrile hydratases found in databases or hiding in metagenomic libraries, simple and efficient high-throughput assays are necessary. For that purpose, several assays based on three different principles have been developed (217). The first group uses indicators like bromothymol blue, bromocresol purple, or phenol red to measure a pH shift caused by the carboxylic acid or the ammonia released during nitrile degradation $(122,218,219)$. These methods are easy to perform and the respective color shifts can be detected without additional equipment. However, their success depends on the strength of the released acid. In case of acids equally strong as the basic ammonia, no $\mathrm{pH}$ shift will occur and therefore prevent the detection of nitrile-degrading enzymes. The second group of highthroughput assays focuses on the released carboxylic acids $(220,221)$. Due to the structural diversity of carboxylic acids, these methods are often limited to specific molecules, making the screening of broad substrate libraries impossible. The third group of assays detects the ammonia released by nitrilase reactions. Besides screenings based on the established Berthelot reaction (217), a fast and simple assay using $\mathrm{CoCl}_{2}$ as indicator is published (222). Drawback of the first is the use of highly toxic phenol hypochlorite mixtures, whereas the second is not sensitive enough for detection of low amounts of ammonia.

To assist functional screening of large amounts of putative nitrile-degrading enzymes, a novel nitrilase high-throughput assays was developed during the here presented work (Chapter 3.3). It is based on well-established $\mathrm{NAD}(\mathrm{P}) \mathrm{H}$-coupled systems, which monitor the conversion of $\mathrm{NAD}(\mathrm{P}) \mathrm{H}$ to $\mathrm{NAD}(\mathrm{P})^{+}$or vice versa (223-226). In case of nitrilases, the released ammonia is converted by a glutamate dehydrogenase $(\mathrm{GDH})$ to glutamate (Figure 2 ). This reaction involves the oxidation of NADH to NAD and is used to determine nitrile degradation.

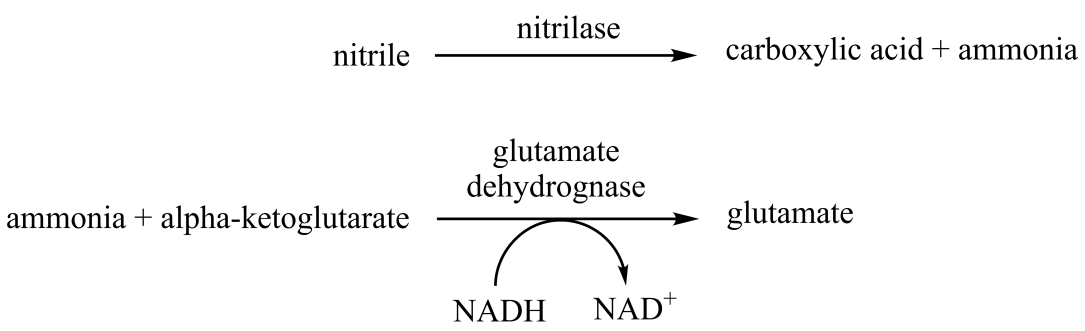

Figure 2: Reactions of a NADH-coupled nitrilase assay. 
In contrast to a previously published NADH-coupled nitrilase assay (132), the novel approach does not depend on long incubation times. For that purpose, nitrile degradation and NADH conversion were coupled in a single mixture. By using a plate reader, real-time conversion of the cofactor is monitored in 96-well plates, providing direct insights into nitrilase efficiency for different substrates. Furthermore, the assay was performed with crude extract containing the overexpressed putative enzymes, allowing the simple and fast screening of large (meta)genomic libraries.

Unspecific NADH conversion was observed during first tests of the assay with an already characterized nitrilase. Most likely, unintended NADH oxidation by other proteins of the crude extract caused NAD ${ }^{+}$ formation. For example, members of the tricarboxylic acid (TCA) cycle like malate dehydrogenases can also convert $\mathrm{NADH}$ to $\mathrm{NAD}^{+}$and therefore influence the obtained signal (227). Consequently, a sharp threshold is recommended to reduce number of false positives. In addition, every reaction exhibited a turning point after which the optical density was increasing. This observation was independent of the used substrate and most likely caused by the reverse reaction of $\mathrm{NAD}^{+}$to $\mathrm{NADH}$. In consequence, this assay can lead to wrong conclusions when performed with single measurements. It is not possible to differentiate between nitrilase activity, side reactions of the crude extract, and regeneration of the cofactor for a single data point. Only the full reaction curve obtained by continuous measurements with a fast plate reader allows assessments of nitrile degradation.

Overall, the here presented workflow allows the simple and fast verification of putative nitrilases identified during sequence-based screenings. Optimization of the bioinformatic analyses could further improve the outcome of nitrilase screenings and advance identification of novel biocatalysts. During in silico analyses of the 70 putative nitrilases used as input, almost $50 \%$ of the enzymes were excluded from further analysis due to their low quality. Still, only putative enzymes clustering with characterized nitrilases obtained from SWISS-Prot showed enzymatic activity. Therefore, it can be recommended to discard all "unique" putative enzymes when time restrictions apply or the number of putative nitrilases is too large for handling. Nonetheless, it has to be considered that this additional filtering will also reduce the chance for identification of truly novel enzymes. Furthermore, prediction of substrate specificity based on sequence homology could help to identify possible genes of interest before wet lab work even starts. Such an approach was already published with focus on dinitriles and led to promising results (228). In combination with the novel high-throughput screening, identification of nitrilases of interest could be significantly improved and support the increasing industrial demand for these biocatalysts. 


\subsection{Characterization of a novel arylacetonitrilase}

Although nitrilases are an important class of enzymes for various industrial processes, only a small number has been characterized. Curated enzyme databases like SWISS-Prot contain thousands of phosphatases, esterases, or lipases, but less than 100 nitrilases. Relationship between sequence, structure, and function is nearly unknown because of the low number of characterized nitrilases (75). Consequently, one of the nitrilases identified with the novel high-throughput assay was characterized in detail to support knowledge and understanding of these enzymes. Due to its unique substrate specificity, the phenylacetonitrile-degrading nitrilase was chosen for this task (Chapter 3.3).

Table 3: Properties of characterized bacterial arylacetonitrilases. Mass refers to mass of homomers; activity refers to specific activity for phenylacetonitrile.

\begin{tabular}{|l|l|l|l|l|l|}
\hline Origin & $\begin{array}{l}\mathbf{p H} \\
\text { optimum }\end{array}$ & $\begin{array}{l}\text { Temperature } \\
\text { optimum }\left[{ }^{\circ} \mathbf{C}\right]\end{array}$ & $\begin{array}{l}\text { Mass } \\
{[\mathbf{k D a}]}\end{array}$ & $\begin{array}{l}\text { Activity } \\
{[\mathbf{U} / \mathbf{m g}]}\end{array}$ & References \\
\hline Alcaligenes faecalis JM3 & 7.5 & 45 & 44 & 85.0 & $(229)$ \\
\hline Alcaligenes faecalis ATCC 8750 & 7.5 & $40-45$ & 32 & 30.0 & $(230)$ \\
\hline Alcaligenes faecalis ZJUTB10 & 7.5 & 40 & 44 & 41.9 & $(231)$ \\
\hline Alcaligenes sp. MTCC 10675 & 6.5 & 50 & 60 & 65 & $(232)$ \\
\hline Bradyrhizobium japonicum USDA110 & - & - & 37 & 5.3 & $(233)$ \\
\hline Luminiphilus syltensis NOR5-1B & 7.0 & 40 & 43 & 89.2 & $(234)$ \\
\hline Pseudomonas fluorescens EBC191 & $6.0-6.5$ & $50-55$ & 38 & 67.7 & $(235)$ \\
\hline Pseudomonas putida MTCC 5110 & 7.0 & 40 & 43 & 11.2 & $(78)$ \\
\hline Compost metagenome & 6.0 & 50 & 36 & 13.9 & This study \\
\hline
\end{tabular}

Based on its substrate specificity, the novel nitrilase belongs to the class of arylacetonitrilases (EC 3.5.5.5). Arylacetonitrilases are probably the industrially most imported group of nitrile-degrading enzymes as they allow the conversion of mandelonitrile to mandelic acid (110). With less than 10 respective entries in databases like SWISS-Prot, they are the smallest subgroup of nitrilases and to the best of my knowledge, only Alcaligenes, Bradyrhizobium, Luminiphilus, and Pseudomonas contain characterized bacterial arylacetonitrilases (Table 3). Consequently, several studies with focus on understanding and optimization of these enzymes have been performed (236-241). Nevertheless, identification of novel arylacetonitrilases will provide new opportunities and insights into sequencefunction relationships that cannot be easily obtained by other experiments. For example, based on protein size as well as $\mathrm{pH}$ and temperature optimum, the here characterized enzyme is closely related to the arylacetonitrilases of $B$. japonicum USDA110 and P. fluorescens EBC191 (Table 4). These results are further supported by sequence similarity, as the respective enzymes build a separate branch of arylacetonitrilases (Figure 4). In contrast, the overall substrate specificity of the novel arylacetonitrilase is similar to that of $P$. putida MTCC 5110, although their sequences are not closely related. 


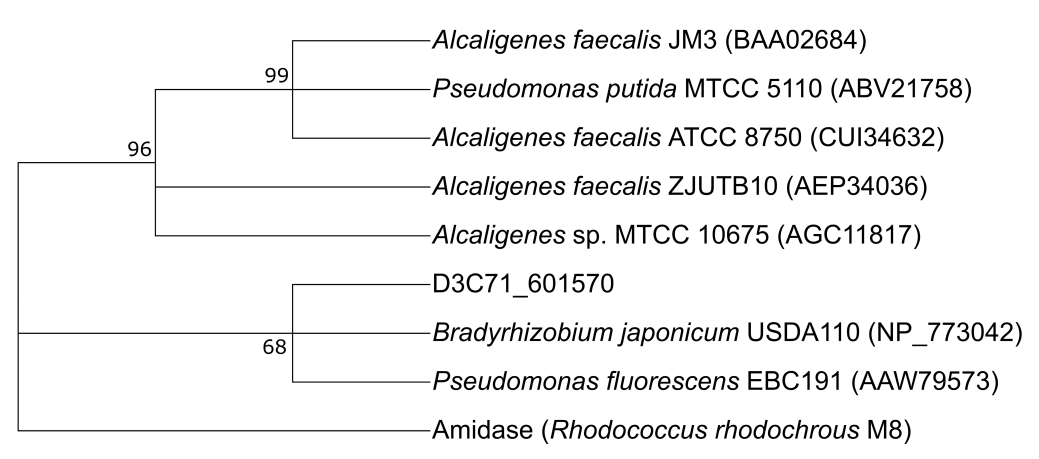

Figure 4: Phylogenetic tree containing all identified novel nitrilases. 500 bootstrap replicates were calculated and the tree was condensed at values below $50 \%$. No nitrilase sequence was available for Luminiphilus syltensis NOR5-1B. WAG algorithm including gamma sites was chosen for calculation of the phylogenetic tree (242).For general parameters and references for $R$. rhodochrous M8 amidase, see material and methods section of chapter 3.1. NCBI accession number for protein sequences are in brackets, for references see Table 3 .

Besides its unusual sequence-function relationship, the novel nitrilase exhibits other non-common features like optimum activity at high temperatures and over a broad $\mathrm{pH}$ range. Furthermore, it exhibits a unique long-term stability as more than $80 \%$ of initial activity remained after two weeks of storage. In contrast, arylacetonitrilases of $A$. faecalis JM3 and $P$. fluorescens EBC191 showed only 40\% and $25 \%$ remaining activity after one or two weeks under similar conditions, respectively $(229,235)$. In conclusion, the here identified and characterized nitrilase leads to a better understanding of sequence-function relationship of these rare and valuable enzymes and is an interesting candidate for industrial applications.

\subsection{A novel (meta)transcriptomic screening approach}

Screening for novel enzymes is nowadays often done by function- or sequence-based metagenomic approaches (see Chapter 2.7). Both have their specific drawbacks such as problems due to expression in heterologous hosts or inability to identify truly novel enzymes. In addition, they cannot identify complex networks sometimes involved in metabolization of a substrate (243).

Differential transcriptomic approaches are without these inherent limitations. Microbial cultures are grown under different conditions and their transcriptomes are isolated and sequenced. Comparison of the expression profiles highlights genes up- and downregulated for each treatment, leading to identification of genes encoding enzymes or pathways specific for the tested conditions. Although this approach seems to be promising for identification of novel biocatalysts, most research focuses on clinical aspects like human diseases or different pathogens (244-252). In contrast, only a few studies with focus on industrial topics such as increased solvent tolerance of living cells or identification of novel biocatalysts have been performed $(136,253-255)$. 
Important for differential transcriptomics is proper normalization of the transcriptomes. Without these steps during data analysis, obtained results could be misleading or wrong. Consequently, different approaches for normalization of transcriptomic data have been developed and were extensively reviewed (256-259). Several of these methods depend on count-based normalization, but compensate only for differences in sequencing depth (256). When comparing two conditions with equal strength in gene expression but double the number of expressed genes in the first condition, these approaches will introduce a severe bias. After total count normalization, only half the number of reads per gene will be assigned in the first treatment, distorting the outcome of the analysis (260). To account for this problem, new statistical models have been proposed. They assume that most genes between two samples are not differentially expressed and calculate a correction factor for all read counts to fulfill this hypothesis (256). Most prominent implementations of this approach can be found in edgeR (261) and DESeq2 (262) and both methods are nowadays recommended for transcriptome analysis $(256,257)$.

When trying to apply standard transcriptome normalization methods like DESeq2 to metatranscriptomes, a major problem occurs. During work with a single strain, abundance of this organism in the culture is always $100 \%$. In contrast, abundance of organisms can significantly change in complex communities between two treatments (263-265), as also found in the here presented study. If an organism would be absent during first treatment and present during second, all its genes would appear upregulated under the second treatment during subsequent analysis. To prevent this community-based bias, an additional normalization step is necessary (266). For that purpose, the metagenomic backbone must be divided into bins consisting of single species. Subsequently, transcriptomic reads can be assigned to their biological origin and are individually normalized for each bin. Afterwards, the normalized reads can be combined to the final metatranscriptomic dataset and analyzed with algorithms like DESeq2. To the best of my knowledge, the here presented metatranscriptomic study is the first using this thorough normalization approach. Previous studies dealing with differential metatranscriptomics did not normalize their data based on community composition and may therefore contain erroneous results or assumptions (266-273).

To establish the novel transcriptome-based screening approach, acetonitrile was used as substrate of choice (Chapter 3.4). During previous toxicity analysis (Chapter 3.1), no growth-suppression was observed even at $25 \mathrm{mM}$ concentration. Furthermore, cultures treated with acetonitrile exhibited high diversity, indicating no toxic effect on many microorganisms. In conclusion, unintended expression of stress response genes should be minimal for this compound when compared to other tested nitriles. For a proof of principle, the previously isolated acetonitrile-degrading Rhodococcus erythropolis ACN1 (Chapter 3.2) was used. When comparing the transcriptomes of R. erythropolis ACN1 after treatment with acetonitrile or acetic acid and ammonia, 23 differentially expressed genes were identified. 
Apparently, acetonitrile has a minor effect on general gene expression and is therefore a promising substrate for metatranscriptome-based nitrilase screenings. In addition, only four of the differentially expressed genes were upregulated in the acetonitrile-containing sample. Most likely all of them are involved in nitrile degradation, demonstrating the potential of the tested setup.

Analysis of the metatranscriptomes revealed many differentially expressed genes. Comparing the R. erythropolis ACN1 transcriptome with the metatranscriptome, number of differentially expressed genes per species increased from 23 (4 up- and 19 downregulated) to 113 (72 up- and 41 downregulated). Presumably, cross-feeding and metabolic networks across different community members contribute to these differences (274-277). Although these effects could hinder the fast identification of novel biocatalysts, they allow insights into microbial interaction. In addition, the here presented approach may increase knowledge on resistance mechanisms. An example can be seen for Cupriavidus (species \#3) with several upregulated cytochrome c-related genes. Nitrile-induced inhibition of cytochrome c oxidases has been proposed for eukaryotes (Chapter 2.3), but mode of action in prokaryotes is unknown. The metatranscriptomic data solidify assumptions on similar mechanisms in bacteria as overexpression of these essential genes may help to compensate the inhibition. In addition to putative resistance mechanisms, a highly upregulated nitrilase-encoding gene was identified. It can be assumed that this enzyme acts on acetonitrile, making it the first nitriledegrading biocatalyst discovered by a transcriptomic approach. Nevertheless, further characterization of the respective enzyme is necessary to confirm this assumption. For that purpose, the previously established high-throughput assay can be used (Chapter 3.3), as it would allow the fast and simple screening of the putative nitrilase with several substrates.

In conclusion, the here presented screening demonstrates the potential of differential metatranscriptomics. Although first analysis revealed just two enzymes involved in nitrile degradation, valuable insights into cross-feeding and putative resistance mechanisms were obtained. In addition, analysis of upregulated hypothetical proteins and screening with a high-throughput nitrilase assay could lead to identification of novel biocatalysts. Screening of nitrilases was challenging due to toxicity of the substrate, but should be simple for other compounds like lipids, alcohols, or esters. Therefore, this approach might lead to identification of several novel classes of biocatalysts and is a promising method to support the demands and goals of green chemistry. 
Chapter V

General references 


\section{General references}

1. Berzelius JJ. 1835. Årsberättelse om framsteg i fysik och kemi. Royal Swedish Academy of Sciences, Stockholm, Sweden.

2. Dulong PL, Thénard L. 1823. Note sur la propriété que possèdent quelques métaux de faciliter la combinaison des fluides élastiques. Ann Chim Phys 23:440-444.

3. Dulong PL, Thénard L. 1823. Nouvelles observations sur la propriété dont jouissent certains corps de favoriser la combinaison des fluides élastiques. Ann Chim Phys 24:380-387.

4. Appl M. 2006. Ammonia., p. 11-13. In Appl, M (ed.), Ullmann's encyclopedia of industrial chemistry, 1st ed. Wiley-VCH, Weinheim, Germany.

5. Lindström B, Pettersson L. 2003. A brief history of catalysis. CATTECH 7:130-138.

6. Wohlgemuth R. 2010. Biocatalysis - key to sustainable industrial chemistry. Curr Opin Biotechnol 21:713-724.

7. Clouthier CM, Pelletier JN. 2012. Expanding the organic toolbox: a guide to integrating biocatalysis in synthesis. Chem Soc Rev 41:1585-1605.

8. Baran PS, Maimone TJ, Richter JM. 2007. Total synthesis of marine natural products without using protecting groups. Nature 446:404-408.

9. Pfromm PH. 2017. Towards sustainable agriculture: Fossil-free ammonia. J Renew Sustain Energy 9:034702.

10. Karp EM, Eaton TR, Sànchez i Nogué V, Vorotnikov V, Biddy MJ, Tan ECD, Brandner DG, Cywar RM, Liu R, Manker LP, Michener WE, Gilhespy M, Skoufa Z, Watson MJ, Fruchey OS, Vardon DR, Gill RT, Bratis AD, Beckham GT. 2017. Renewable acrylonitrile production. Science 358:13071310.

11. Chauvel A, Lefebvre G. 1989. Petrochemical processes 2. Major oxygenated, chlorinated and nitrated derivatives., 2nd ed. Imprimerie Nouvelle, Saint-Jean-de-Braye, France.

12. Sheldon RA. 2018. Metrics of green chemistry and sustainability: Past, present, and future. ACS Sustain Chem Eng 6:32-48.

13. United States Environmental Protection Agency. 1990. Pollution Prevention Act of 1990.

14. Anastas PT, Warner JC. 1998. Green chemistry: Theory and practice., 1st ed. Oxford University Press, Oxford, UK.

15. Grasselli RK, Trifirò F. 2016. Acrylonitrile from biomass: Still far from being a sustainable process. Top Catal 59:1651-1658.

16. Sheldon RA. 2016. Biocatalysis and Green Chemistry, p. 1-15. In Patel, RN (ed.), Green biocatalysis, 1st ed. John Wiley \& Sons, Hoboken, NJ, USA.

17. Pasteur L. 1858. Mémoire sur la fermentation de l'acide tartrique. C R Hebd Seances Acad Sci 46:615-618. 
18. Buchholz KB, Poulsen PB. 2000. Overview of history of applied biocatalysis., p. 1-17. In Straathof, AJJ, Adlercreutz, P (eds.), Applied biocatalysis, 2nd ed. Harwood Academic Publishers, Amsterdam, Netherlands.

19. Buchner E. 1897. Alkoholische Gährung ohne Hefezellen. Berichte der Dtsch Chem Gesellschaft 30:117-124.

20. Reetz MT. 2013. Biocatalysis in organic chemistry and biotechnology: Past, present, and future. J Am Chem Soc 135:12480-12496.

21. Bornscheuer UT, Buchholz KB. 2005. Highlights in biocatalysis - Historical landmarks and current trends. Eng Life Sci 5:309-323.

22. Glick BR, Pasternak JJ, Patten CL. 2009. Molecular biotechnology: Principles and applications of recombinant DNA., 4th ed. ASM Press, Washington, DC, USA.

23. Lutz S, Bornscheuer UT. 2009. Protein engineering handbook., 1st ed. Wiley-VCH, Weinheim, Germany.

24. Bornscheuer UT, Huisman GW, Kazlauskas RJ, Lutz S, Moore JC, Robins K. 2012. Engineering the third wave of biocatalysis. Nature 485:185-194.

25. Hudlicky T, Reed JW. 2009. Applications of biotransformations and biocatalysis to complexity generation in organic synthesis. Chem Soc Rev 38:3117-3132.

26. Sun H, Zhang H, Ang EL, Zhao H. 2018. Biocatalysis for the synthesis of pharmaceuticals and pharmaceutical intermediates. Bioorg Med Chem 26:1275-1284.

27. Patel RN. 2008. Synthesis of chiral pharmaceutical intermediates by biocatalysis. Coord Chem Rev 252:659-701.

28. Fernandes P. 2010. Enzymes in food processing: A condensed overview on strategies for better biocatalysts. Enzyme Res 2010:862537.

29. BCC Research Staff. 2018. Global markets for enzymes in industrial applications. Wellesley, MA, USA.

30. Ansari MI, Malik A. 2007. Biosorption of nickel and cadmium by metal resistant bacterial isolates from agricultural soil irrigated with industrial wastewater. Bioresour Technol 98:31493153.

31. Banik S, Das K, Islam M, Salimullah M. 2014. Recent advancements and challenges in microbial bioremediation of heavy metals contamination. JSM Biotechnol Biomed Eng 2:1035.

32. Francis A, Nancharaiah Y. 2015. In situ and ex situ bioremediation of radionuclide-contaminated soils at nuclear and norm sites., p. 185-236. In van Velzen, L (ed.), Environmental remediation and restoration of contaminated nuclear and norm sites, 1st ed. Woodhead Publishing, Sawston, UK.

33. Prakash D, Gabani P, Chandel AK, Ronen Z, Singh O V. 2013. Bioremediation: A genuine technology to remediate radionuclides from the environment. Microb Biotechnol 6:349-360.

34. Ito T, Adachi Y, Yamanashi Y, Shimada Y. 2016. Long-term natural remediation process in textile dye-polluted river sediment driven by bacterial community changes. Water Res 100:458-465. 
35. Vikrant K, Giri BS, Raza N, Roy K, Kim K-H, Rai BN, Singh RS. 2018. Recent advancements in bioremediation of dye: Current status and challenges. Bioresour Technol 253:355-367.

36. Deshkar A, Dhamorikar N, Godbole S, Krishnamurthi K, Saravanadevi S, Vijay R, Kaul S, Chakrabarti T. 2003. Bioremediation of soil contaminated with organic compounds with special reference to acrylonitrile. Ann Chim 93:729-737.

37. Baxter J, Garton NJ, Cummings SP. 2006. The impact of acrylonitrile and bioaugmentation on the biodegradation activity and bacterial community structure of a topsoil. Folia Microbiol (Praha) 51:591-597.

38. Prasad S, Bhalla TC. 2010. Nitrile hydratases (NHases): At the interface of academia and industry. Biotechnol Adv 28:725-741.

39. Wray V, Davis RH, Nahrstedt A. 1983. Biosynthesis of cyanogenic glycosides in butterflies and moths: Incorporation of valine and isoleucine into linamarin and lotaustralin by Zygaena and Heliconius species (Lepidoptera). Zeitschrift für Naturforsch C 38:583-588.

40. Conn EE. 1979. Biosynthesis of cyanogenic glycosides. Naturwissenschaften 66:28-34.

41. Blum MS, Woodring JP. 1962. Secretion of benzaldehyde and hydrogen cyanide by the millipede Pachydesmus crassicutis (Wood). Science 138:512-513.

42. Moore BP. 1967. Hydrogen cyanide in the defensive secretions of larval Paropsini (Coleoptera: Chrysomelidae). Aust J Entomol 6:36-38.

43. Fulmor W, Van Lear GE, Morton GO, Mills RD. 1970. Isolation and absolute configuration of the aeroplysinin I enantiomorphic pair from lanthella ardis. Tetrahedron Lett 11:4551-4552.

44. Singh SK, Gurusiddaiah S, Whalen JW. 1985. Treponemycin, a nitrile antibiotic active against Treponema hyodysenteriae. Antimicrob Agents Chemother 27:239-245.

45. Shentu X-P, Cao Z-Y, Xiao Y, Tang G, Ochi K, Yu X-P. 2018. Substantial improvement of toyocamycin production in Streptomyces diastatochromogenes by cumulative drug-resistance mutations. PLoS One 13:e0203006.

46. Strobel GA. 1966. The fixation of hydrocyanic acid by a psychrophilic basidiomycete. J Biol Chem 241:2618-2621.

47. Gasco A, Serafino A, Mortarini V, Menziani E, Bianco MA, Ceruti Scurti J. 1974. An antibacterial and antifungal compound from Calvatia lilacina. Tetrahedron Lett 15:3431-3432.

48. Tapper BA, Macdonald MA. 1974. Cyanogenic compounds in cultures of a psychrophilic basidiomycete (snow mold). Can J Microbiol 20:563-566.

49. Gleadow RM, Møller BL. 2014. Cyanogenic glycosides: Synthesis, physiology, and phenotypic plasticity. Annu Rev Plant Biol 65:155-185.

50. Paetz W, Nass G. 1973. Biochemical and immunological characterization of threonyl-tRNA synthetase of two borrelidin-resistant mutants of Escherichia coli K12. Eur J Biochem 35:331337.

51. García-Vilas JA, Martínez-Poveda B, Quesada AR, Medina MÁ. 2016. Aeroplysinin-1, a spongederived multi-targeted bioactive marine drug. Mar Drugs 14:1. 
52. Fürstenberg-Hägg J, Zagrobelny M, Bak S. 2013. Plant defense against insect herbivores. Int J Mol Sci 14:10242-10297.

53. Brückner A, Raspotnig G, Wehner K, Meusinger R, Norton RA, Heethoff M. 2017. Storage and release of hydrogen cyanide in a chelicerate (Oribatula tibialis). Proc Natl Acad Sci U S A 114:3469-3472.

54. Selmar D, Lieberei R, Biehl B. 1988. Mobilization and utilization of cyanogenic glycosides: The linustatin pathway. Plant Physiol 86:711-716.

55. Tanii H, Hashimoto K. 1984. Studies on the mechanism of acute toxicity of nitriles in mice. Arch Toxicol 55:47-54.

56. Grogan J, DeVito SC, Pearlman RS, Korzekwa KR. 1992. Modeling cyanide release from nitriles: Prediction of cytochrome P450 mediated acute nitrile toxicity. Chem Res Toxicol 5:548-552.

57. Uetrecht JP, Trager W. 2007. Oxidation pathways and the enzymes that mediate them., p. 33108. In Uetrecht, JP, Trager, W (eds.), Drug metabolism: Chemical and enzymatic aspects, 1st ed. Informa Healthcare, New York, NY, USA.

58. Tanii H. 2017. Allyl nitrile: toxicity and health effects. J Occup Health 59:104-111.

59. Willhite CC, Smith RP. 1981. The role of cyanide liberation in the acute toxicity of aliphatic nitriles. Toxicol Appl Pharmacol 59:589-602.

60. Lewis DFV, Wiseman A. 2005. A selective review of bacterial forms of cytochrome P450 enzymes. Enzyme Microb Technol 36:377-384.

61. Richter O-MH, Ludwig B. 2003. Cytochrome c oxidase - Structure, function, and physiology of a redox-driven molecular machine., p. 47-74. In Amara, S, Bamberg, E, Blaustein, M, Grunicke, H, Jahn, R, Lederer, W, Miyajima, A, Murer, H, Offermanns, S, Pfanner, N, Schultz, G, Schweiger, $M$ (eds.), Reviews of physiology, biochemistry and pharmacology, 1st ed. Springer Berlin Heidelberg, Berlin, Germany.

62. Hashimoto K, Kanai R. 1965. Studies on the toxicology of acrylonitrile. Ind Health 3:30-46.

63. Johannsen FR, Levinskas GJ. 1986. Relationships between toxicity and structure of aliphatic nitriles. Fundam Appl Toxicol 7:690-697.

64. Gupta N, Balomajumder C, Agarwal VK. 2010. Enzymatic mechanism and biochemistry for cyanide degradation: A review. J Hazard Mater 176:1-13.

65. Martínková L, Veselá AB, Rinágelová A, Chmátal M. 2015. Cyanide hydratases and cyanide dihydratases: Emerging tools in the biodegradation and biodetection of cyanide. Appl Microbiol Biotechnol 99:8875-8882.

66. Yanase H, Sakamoto A, Okamoto K, Kita K, Sato Y. 2000. Degradation of the metal-cyano complex tetracyanonickelate (II) by Fusarium oxysporum N-10. Appl Microbiol Biotechnol 53:328-334.

67. Benedik MJ, Sewell BT. 2018. Cyanide-degrading nitrilases in nature. J Gen Appl Microbiol 64:90-93. 
68. Jandhyala D, Berman M, Meyers PR, Sewell BT, Willson RC, Benedik MJ. 2003. CynD, the cyanide dihydratase from Bacillus pumilus: Gene cloning and structural studies. Appl Environ Microbiol 69:4794-4805.

69. Luque-Almagro VM, Cabello P, Sáez LP, Olaya-Abril A, Moreno-Vivián C, Roldán MD. 2018. Exploring anaerobic environments for cyanide and cyano-derivatives microbial degradation. Appl Microbiol Biotechnol 102:1067-1074.

70. Pace HC, Brenner C. 2001. The nitrilase superfamily: Classification, structure and function. Genome Biol 2:reviews0001.1.

71. Chen J, Zheng R-C, Zheng Y-G, Shen Y-C. 2009. Microbial transformation of nitriles to high-value acids or amides. Adv Biochem Eng Biotechnol 113:33-77.

72. O'Reilly C, Turner PD. 2003. The nitrilase family of CN hydrolysing enzymes - A comparative study. J Appl Microbiol 95:1161-1174.

73. Gong J-S, Shi J-S, Lu Z-M, Li H, Zhou Z-M, Xu Z-H. 2017. Nitrile-converting enzymes as a tool to improve biocatalysis in organic synthesis: Recent insights and promises. Crit Rev Biotechnol 37:69-81.

74. Gong J-S, Lu Z-M, Li H, Shi J-S, Zhou Z-M, Xu Z-H. 2012. Nitrilases in nitrile biocatalysis: Recent progress and forthcoming research. Microb Cell Fact 11:142.

75. Kaushik S, Mohan U, Banerjee U. 2012. Exploring residues crucial for nitrilase function by site directed mutagenesis to gain better insight into sequence-function relationships. Int J Biochem Mol Biol 3:384-391.

76. Dennett G, Blamey JM. 2016. A new thermophilic nitrilase from an antarctic hyperthermophilic microorganism. Front Bioeng Biotechnol 4:5.

77. Kobayashi M, Yanaka N, Nagasawa T, Yamada H. 1990. Purification and characterization of a novel nitrilase of Rhodococcus rhodochrous K22 that acts on aliphatic nitriles. J Bacteriol 172:4807-4815.

78. Banerjee A, Kaul P, Banerjee UC. 2006. Purification and characterization of an enantioselective arylacetonitrilase from Pseudomonas putida. Arch Microbiol 184:407-418.

79. Gradley ML, Deverson CJF, Knowles CJ. 1994. Asymmetric hydrolysis of R-(-),S(+)-2methylbutyronitrile by Rhodococcus rhodochrous NCIMB 11216. Arch Microbiol 161:246-251.

80. Zheng Y-G, Chen J, Liu Z-Q, Wu M-H, Xing L-Y, Shen Y-C. 2008. Isolation, identification and characterization of Bacillus subtilis ZJB-063, a versatile nitrile-converting bacterium. Appl Microbiol Biotechnol 77:985-993.

81. He Y-C, Xu J-H, Su J-H, Zhou L. 2010. Bioproduction of glycolic acid from glycolonitrile with a new bacterial isolate of Alcaligenes sp. ECU0401. Appl Biochem Biotechnol 160:1428-1440.

82. Ramteke PW, Maurice NG, Joseph B, Wadher BJ. 2013. Nitrile-converting enzymes: An ecofriendly tool for industrial biocatalysis. Biotechnol Appl Biochem 60:459-481.

83. Marron AO, Akam M, Walker G. 2012. Nitrile hydratase genes are present in multiple eukaryotic supergroups. PLoS One 7:e32867. 
84. Huang W, Jia J, Cummings J, Nelson M, Schneider G, Lindqvist Y. 1997. Crystal structure of nitrile hydratase reveals a novel iron centre in a novel fold. Structure 5:691-699.

85. Nakasako M, Odaka M, Yohda M, Dohmae N, Takio K, Kamiya N, Endo I. 1999. Tertiary and quaternary structures of photoreactive Fe-type nitrile hydratase from Rhodococcus sp. N-771: Roles of hydration water molecules in stabilizing the structures and the structural origin of the substrate specificity of the enzyme. Biochemistry 38:9887-9898.

86. Xia Y, Cui W, Liu Z, Zhou L, Cui Y, Kobayashi M, Zhou Z. 2016. Construction of a subunit-fusion nitrile hydratase and discovery of an innovative metal ion transfer pattern. Sci Rep 6:19183.

87. Miyanaga A, Fushinobu S, Ito K, Wakagi T. 2001. Crystal structure of cobalt-containing nitrile hydratase. Biochem Biophys Res Commun 288:1169-1174.

88. Payne MS, Wu S, Fallon RD, Tudor G, Stieglitz B, Turner IM, Nelson MJ. 1997. A stereoselective cobalt-containing nitrile hydratase. Biochemistry 36:5447-5454.

89. Nelp MT, Astashkin A V, Breci LA, McCarty RM, Bandarian V. 2014. The alpha subunit of nitrile hydratase is sufficient for catalytic activity and post-translational modification. Biochemistry 53:3990-3994.

90. Weber BW, Kimani SW, Varsani A, Cowan DA, Hunter R, Venter GA, Gumbart JC, Sewell BT. 2013. The mechanism of the amidases: Mutating the glutamate adjacent to the catalytic triad inactivates the enzyme due to substrate mispositioning. J Biol Chem 288:28514-28523.

91. Sharma M, Sharma NN, Bhalla TC. 2013. Purification studies on a thermo-active amidase of Geobacillus pallidus BTP-5x MTCC 9225 isolated from thermal springs of Tatapani (Himachal Pradesh). Appl Biochem Biotechnol 169:1-14.

92. Agarkar VB, Kimani SW, Cowan DA, Sayed MF-R, Sewell BT. 2006. The quaternary structure of the amidase from Geobacillus pallidus RAPc8 is revealed by its crystal packing. Acta Crystallogr Sect F Struct Biol Cryst Commun 62:1174-1178.

93. Scotto d'Abusco A, Casadio R, Tasco G, Giangiacomo L, Giartosio A, Calamia V, Di Marco S, Chiaraluce R, Consalvi V, Scandurra R, Politi L. 2005. Oligomerization of Sulfolobus solfataricus signature amidase is promoted by acidic $\mathrm{pH}$ and high temperature. Archaea 1:411-423.

94. Bhalla TC, Kumar V, Kumar V, Thakur N, Savitri. 2018. Nitrile metabolizing enzymes in biocatalysis and biotransformation. Appl Biochem Biotechnol 185:925-946.

95. Lenz JD, Stohl EA, Robertson RM, Hackett KT, Fisher K, Xiong K, Lee M, Hesek D, Mobashery S, Seifert HS, Davies C, Dillard JP. 2016. Amidase activity of AmiC controls cell separation and stem peptide release and is enhanced by NIpD in Neisseria gonorrhoeae. J Biol Chem 291:1091610933.

96. Nawaz MS, Khan AA, Seng JE, Leakey JE, Siitonen PH, Cerniglia CE. 1994. Purification and characterization of an amidase from an acrylamide-degrading Rhodococcus sp. Appl Environ Microbiol 60:3343-3348.

97. Hirrlinger B, Stolz A, Knackmuss HJ. 1996. Purification and properties of an amidase from Rhodococcus erythropolis MP50 which enantioselectively hydrolyzes 2-arylpropionamides. J Bacteriol 178:3501-3507. 
98. Komeda H, Asano Y. 2000. Gene cloning, nucleotide sequencing, and purification and characterization of the D-stereospecific amino-acid amidase from Ochrobactrum anthropi SV3. Eur J Biochem 267:2028-2035.

99. Ciskanik LM, Wilczek JM, Fallon RD. 1995. Purification and characterization of an enantioselective amidase from Pseudomonas chlororaphis B23. Appl Environ Microbiol 61:9981003.

100. Bray HG, James SP, Raffan IM, Ryman BE, Thorpe W V. 1949. The fate of certain organic acids and amides in the rabbit. 7. An amidase of rabbit liver. Biochem J 44:618-625.

101. Thimann K V, Mahadevan S. 1964. Nitrilase. I. Occurrence, preparation, and general properties of the enzyme. Arch Biochem Biophys 105:133-141.

102. Asano Y, Tani Y, Yamada H. 1980. A new enzyme "nitrile hydratase" which degrades acetonitrile in combination with amidase. Agric Biol Chem 44:2251-2252.

103. Fleming FF, Yao L, Ravikumar PC, Funk L, Shook BC. 2010. Nitrile-containing pharmaceuticals: Efficacious roles of the nitrile pharmacophore. J Med Chem 53:7902-7917.

104. Buchholz K. 2016. A breakthrough in enzyme technology to fight penicillin resistance - Industrial application of penicillin amidase. Appl Microbiol Biotechnol 100:3825-3839.

105. Elander RP. 2003. Industrial production of beta-lactam antibiotics. Appl Microbiol Biotechnol 61:385-392.

106. Yamamoto K, Komatsu K. 1991. Purification and characterization of nitrilase responsible for the enantioselective hydrolysis from Acinetobacter sp. AK 226. Agric Biol Chem 55:1459-1466.

107. Kaul P, Banerjee A, Banerjee UC. 2007. Nitrile hydrolases., p. 531-547. In Polaina, J, MacCabe, AP (eds.), Industrial enzymes. Structure, function and applications., 1st ed. Springer Netherlands, Dordrecht, Netherlands.

108. Asano Y. 2015. Hydrolysis of nitriles and amides., p. 255-276. In Faber, K, Fessner, W-D, Turner, N (eds.), Biocatalysis in organic synthesis 1, 1st ed. Georg Thieme Verlag, Stuttgart, Germany.

109. Schmidberger JW, Hepworth L, Green AP, Flitsch SL. 2015. Enzymatic synthesis of amides., p. 329-372. In Faber, K, Fessner, W-D, Turner, NJ (eds.), Biocatalysis in organic synthesis 1, 1st ed. Georg Thieme Verlag, Stuttgart, Germany.

110. Debabov VG, Yanenko AS. 2011. Biocatalytic hydrolysis of nitriles. Rev J Chem 1:385-402.

111. Bhalla TC, Savitri. 2016. Vitamin B3, Niacin, p. 41-66. In Vandamme, EJ, Revuelta, JL (eds.), Industrial biotechnology of vitamins, biopigments, and antioxidants, 1st ed. Wiley-VCH, Weinheim, Germany.

112. Chuck R. 2005. Technology development in nicotinate production. Appl Catal A Gen 280:75-82.

113. Holtze MS, Hansen HCB, Juhler RK, Sørensen J, Aamand J. 2007. Microbial degradation pathways of the herbicide dichlobenil in soils with different history of dichlobenil-exposure. Environ Pollut 148:343-351.

114. Baxter J, Cummings SP. 2008. The degradation of the herbicide bromoxynil and its impact on bacterial diversity in a top soil. J Appl Microbiol 104:1605-1616. 
115. Freyssinet G, Pelissier B, Freyssinet M, Delon R. 1996. Crops resistant to oxynils: From the laboratory to the market. F Crop Res 45:125-133.

116. Chapatwala KD, Babu GR V., Armstead ER, White EM, Wolfram JH. 1995. A kinetic study on the bioremediation of sodium cyanide and acetonitrile by free and immobilized cells of Pseudomonas putida. Appl Biochem Biotechnol 51-52:717-726.

117. Dias JCT, Rezende RP, Linardi VR. 2001. Bioconversion of nitriles by Candida guilliermondii CCT 7207 cells immobilized in barium alginate. Appl Microbiol Biotechnol 56:757-761.

118. Zhou XF, Zhang YL, Xu DQ, Cao WH, Dai CM, Qiang ZM, Yang Z, Zhao JF. 2008. Treatment of succinonitrile wastewater by immobilized high efficiency microorganism strains. Water Sci Technol 58:911-918.

119. Li C, Li Y, Cheng X, Feng L, Xi C, Zhang Y. 2013. Immobilization of Rhodococcus rhodochrous BX2 (an acetonitrile-degrading bacterium) with biofilm-forming bacteria for wastewater treatment. Bioresour Technol 131:390-396.

120. Li C, Yue Z, Feng F, Xi C, Zang H, An X, Liu K. 2016. A novel strategy for acetonitrile wastewater treatment by using a recombinant bacterium with biofilm-forming and nitrile-degrading capability. Chemosphere 161:224-232.

121. Jardine JL, Stoychev S, Mavumengwana V, Ubomba-Jaswa E. 2018. Screening of potential bioremediation enzymes from hot spring bacteria using conventional plate assays and liquid chromatography - Tandem mass spectrometry (Lc-Ms/Ms). J Environ Manage 223:787-796.

122. Santoshkumar M, Nayak AS, Anjaneya O, Karegoudar TB. 2010. A plate method for screening of bacteria capable of degrading aliphatic nitriles. J Ind Microbiol Biotechnol 37:111-115.

123. Harwood C, Buckley M. 2008. The uncharted microbial world: Microbes and their activities in the environment., p. 1-37. In Harwood, C, Buckley, M (eds.), American Academy of Microbiology. ASM, Seattle, WA, USA.

124. Parachin NS, Gorwa-Grauslund MF. 2011. Isolation of xylose isomerases by sequence- and function-based screening from a soil metagenomic library. Biotechnol Biofuels 4:9.

125. Cheng J, Romantsov T, Engel K, Doxey AC, Rose DR, Neufeld JD, Charles TC. 2017. Functional metagenomics reveals novel beta-galactosidases not predictable from gene sequences. PLoS One 12:e0172545.

126. Popovic A, Hai T, Tchigvintsev A, Hajighasemi M, Nocek B, Khusnutdinova AN, Brown G, Glinos J, Flick R, Skarina T, Chernikova TN, Yim V, Brüls T, Paslier D Le, Yakimov MM, Joachimiak A, Ferrer M, Golyshina O V., Savchenko A, Golyshin PN, Yakunin AF. 2017. Activity screening of environmental metagenomic libraries reveals novel carboxylesterase families. Sci Rep 7:44103.

127. Soares Bragança CR, Dooley-Cullinane T-M, O’Reilly C, Coffey L. 2017. Applying functional metagenomics to search for novel nitrile-hydrolyzing enzymes using environmental samples. Biomater Tissue Technol 1:1000108.

128. Gabor EM, Alkema WBL, Janssen DB. 2004. Quantifying the accessibility of the metagenome by random expression cloning techniques. Environ Microbiol 6:879-886.

129. Bayer TS, Widmaier DM, Temme K, Mirsky EA, Santi D V, Voigt CA. 2009. Synthesis of methyl halides from biomass using engineered microbes. J Am Chem Soc 131:6508-6515. 
130. Liu SP, Liu RX, Zhang L, Shi GY. 2015. Sequence-based screening and characterization of cytosolic mandelate oxidase using oxygen as electron acceptor. Enzyme Microb Technol 69:2430.

131. Maimanakos J, Chow J, Gaßmeyer SK, Güllert S, Busch F, Kourist R, Streit WR. 2016. Sequencebased screening for rare enzymes: New insights into the world of AMDases reveal a conserved motif and 58 novel enzymes clustering in eight distinct families. Front Microbiol 7:1332.

132. Vergne-Vaxelaire C, Bordier F, Fossey A, Besnard-Gonnet M, Debard A, Mariage A, Pellouin V, Perret A, Petit J-L, Stam M, Salanoubat M, Weissenbach J, De Berardinis V, Zaparucha A. 2013. Nitrilase activity screening on structurally diverse substrates: Providing biocatalytic tools for organic synthesis. Adv Synth Catal 355:1763-1779.

133. Streit WR, Daniel R. 2017. Metagenomics., 2nd ed. Springer New York, New York, NY, USA.

134. Tripathy S, Padhi SK, Sen R, Mohanty S, Samanta M, Maiti NK. 2016. Profiling of Brevibacillus borstelensis transcriptome exposed to high temperature shock. Genomics 107:33-39.

135. Erickson KE, Otoupal PB, Chatterjee A. 2017. Transcriptome-level signatures in gene expression and gene expression variability during bacterial adaptive evolution. mSphere 2:e00009-17.

136. Yang J-W, Zheng D-J, Cui B-D, Yang M, Chen Y-Z. 2016. RNA-seq transcriptome analysis of a Pseudomonas strain with diversified catalytic properties growth under different culture medium. Microbiologyopen 5:626-636.

137. Alongi DM. 1994. The role of bacteria in nutrient recycling in tropical mangrove and other coastal benthic ecosystems. Hydrobiologia 285:19-32.

138. Tanner AC. 1985. The role of bacteria in the cycling of nutrients within the maritime antarctic environment., p. 123-127. In Siegfried, W, Condy, P, Laws, R (eds.), Antarctic nutrient cycles and food webs, 1st ed. Springer Berlin Heidelberg, Berlin, Germany.

139. Rashid MI, Mujawar LH, Shahzad T, Almeelbi T, Ismail IMI, Oves M. 2016. Bacteria and fungi can contribute to nutrients bioavailability and aggregate formation in degraded soils. Microbiol Res 183:26-41.

140. Sahu N, Vasu D, Sahu A, Lal N, Singh SK. 2017. Strength of microbes in nutrient cycling: A key to soil health., p. 69-86. In Meena, VS, Mishra, PK, Bisht, JK, Pattanayak, A (eds.), Agriculturally important microbes for sustainable agriculture, 1st ed. Springer Singapore, Singapore.

141. Boyle SA, Yarwood RR, Bottomley PJ, Myrold DD. 2008. Bacterial and fungal contributions to soil nitrogen cycling under Douglas fir and red alder at two sites in Oregon. Soil Biol Biochem 40:443-451.

142. Gougoulias C, Clark JM, Shaw LJ. 2014. The role of soil microbes in the global carbon cycle: tracking the below-ground microbial processing of plant-derived carbon for manipulating carbon dynamics in agricultural systems. J Sci Food Agric 94:2362-2371.

143. Hayatsu M, Tago K, Saito M. 2008. Various players in the nitrogen cycle: Diversity and functions of the microorganisms involved in nitrification and denitrification. Soil Sci Plant Nutr 54:33-45.

144. Kaiser K, Wemheuer B, Korolkow V, Wemheuer F, Nacke H, Schöning I, Schrumpf M, Daniel R. 2016. Driving forces of soil bacterial community structure, diversity, and function in temperate grasslands and forests. Sci Rep 6:33696. 
145. Nacke H, Goldmann K, Schöning I, Pfeiffer B, Kaiser K, Castillo-Villamizar GA, Schrumpf M, Buscot F, Daniel R, Wubet T. 2016. Fine spatial scale variation of soil microbial communities under European Beech and Norway Spruce. Front Microbiol 7:2067.

146. Berkelmann D, Schneider D, Engelhaupt M, Heinemann M, Christel S, Wijayanti M, Meryandini A, Daniel R. 2018. How rainforest conversion to agricultural systems in Sumatra (Indonesia) affects active soil bacterial communities. Front Microbiol 9:2381.

147. Lüneberg K, Schneider D, Siebe C, Daniel R. 2018. Drylands soil bacterial community is affected by land use change and different irrigation practices in the Mezquital Valley, Mexico. Sci Rep 8:1413.

148. Bartram AK, Jiang X, Lynch MDJ, Masella AP, Nicol GW, Dushoff J, Neufeld JD. 2014. Exploring links between $\mathrm{pH}$ and bacterial community composition in soils from the Craibstone Experimental Farm. FEMS Microbiol Ecol 87:403-415.

149. Brockett BFT, Prescott CE, Grayston SJ. 2012. Soil moisture is the major factor influencing microbial community structure and enzyme activities across seven biogeoclimatic zones in western Canada. Soil Biol Biochem 44:9-20.

150. Truu M, Ostonen I, Preem J-K, Lõhmus K, Nõlvak H, Ligi T, Rosenvald K, Parts K, Kupper P, Truu J. 2017. Elevated air humidity changes soil bacterial community structure in the Silver Birch stand. Front Microbiol 8:557.

151. Lin Y-T, Jia Z, Wang D, Chiu C-Y. 2017. Effects of temperature on the composition and diversity of bacterial communities in bamboo soils at different elevations. Biogeosciences 14:48794889.

152. Yan N, Marschner P, Cao W, Zuo C, Qin W. 2015. Influence of salinity and water content on soil microorganisms. Int Soil Water Conserv Res 3:316-323.

153. Geisseler D, Scow KM. 2014. Long-term effects of mineral fertilizers on soil microorganisms - A review. Soil Biol Biochem 75:54-63.

154. Martínez JL. 2017. Effect of antibiotics on bacterial populations: a multi-hierachical selection process. F1000Research 6:51.

155. Banks ML, Kennedy AC, Kremer RJ, Eivazi F. 2014. Soil microbial community response to surfactants and herbicides in two soils. Appl Soil Ecol 74:12-20.

156. Li X, Meng D, Li J, Yin H, Liu H, Liu X, Cheng C, Xiao Y, Liu Z, Yan M. 2017. Response of soil microbial communities and microbial interactions to long-term heavy metal contamination. Environ Pollut 231:908-917.

157. Abbas Z, Akmal M, Khan KS, Fayyaz-ul-Hassan. 2014. Effect of buctril super (bromoxynil) herbicide on soil microbial biomass and bacterial population. Brazilian Arch Biol Technol 57:914.

158. Onyenwoke CA, Simonyan KJ. 2014. Cassava post-harvest processing and storage in Nigeria: $A$ review. African J Agric Res 9:3853-3863.

159. Burns AE, Bradbury JH, Cavagnaro TR, Gleadow RM. 2012. Total cyanide content of cassava food products in Australia. J Food Compos Anal 25:79-82. 
160. Lassila T, Hokkanen J, Aatsinki S-M, Mattila S, Turpeinen M, Tolonen A. 2015. Toxicity of carboxylic acid-containing drugs: The role of acyl migration and CoA conjugation investigated. Chem Res Toxicol 28:2292-2303.

161. Vázquez JA, Durán A, Rodríguez-Amado I, Prieto MA, Rial D, Murado MA. 2011. Evaluation of toxic effects of several carboxylic acids on bacterial growth by toxicodynamic modelling. Microb Cell Fact 10:100.

162. Bolarinwa IF, Oke MO, Olaniyan SA, Ajala AS. 2016. A review of cyanogenic glycosides in edible plants., p. 179-192. In Larramendy, ML, Soloneski, S (eds.), Toxicology - New aspects to this scientific conundrum, 1st ed. IntechOpen, London, UK.

163. Cressey P, Saunders D, Goodman J. 2013. Cyanogenic glycosides in plant-based foods available in New Zealand. Food Addit Contam Part A 30:1946-1953.

164. Dreifuss PA, Wood GE, Roach JAG, Brumley WC, Andrzejewski D, Sphon JA. 1980. Field desorption mass spectrometry of cyanogenic glycosides. Biomed Mass Spectrom 7:201-204.

165. Gleadow RM, Haburjak J, Dunn JE, Conn ME, Conn EE. 2008. Frequency and distribution of cyanogenic glycosides in Eucalyptus L'Hérit. Phytochemistry 69:1870-1874.

166. Wu C-F, Xu X-M, Huang S-H, Deng M-C, Feng A-J, Peng J, Yuan J-P, Wang J-H. 2012. An efficient fermentation method for the degradation of cyanogenic glycosides in flaxseed. Food Addit Contam Part A 29:1085-1091.

167. Bolarinwa IF, Orfila C, Morgan MRA. 2015. Determination of amygdalin in apple seeds, fresh apples and processed apple juices. Food Chem 170:437-442.

168. Barceloux DG. 2009. Cyanogenic foods (cassava, fruit kernels, and cycad seeds). Dis Mon 55:336-352.

169. Conn EE. 1969. Cyanogenic glycosides. J Agric Food Chem 17:519-526.

170. Chassagne D, Crouzet JC, Bayonove CL, Baumes RL. 1996. Identification and quantification of passion fruit cyanogenic glycosides. J Agric Food Chem 44:3817-3820.

171. Bolarinwa IF, Orfila C, Morgan MRA. 2014. Amygdalin content of seeds, kernels and food products commercially-available in the UK. Food Chem 152:133-139.

172. Ballhorn DJ. 2011. Cyanogenic glycosides in nuts and seeds., p. 129-136. In Preedy, VR, Watson, RR, Patel, VB (eds.), Nuts and seeds in health and disease prevention, 1st ed. Academic Press, Cambridge, MA, USA.

173. Panter KE. 2018. Cyanogenic glycoside-containing plants., p. 935-940. In Gupta, RC (ed.), Veterinary toxicology. Basic and clinical principles., 3rd ed. Academic Press, Cambridge, MA, USA.

174. D'Abrosca B, DellaGreca M, Fiorentino A, Monaco P, Previtera L, Simonet AM, Zarrelli A. 2001. Potential allelochemicals from Sambucus nigra. Phytochemistry 58:1073-1081.

175. Sherbrooke WC. 1976. Differential acceptance of toxic jojoba seed (Simmondsia chinensis) by four sonoran desert heteromyid rodents. Ecology 57:596-602.

176. Cheeke PR. 1995. Endogenous toxins and mycotoxins in forage grasses and their effects on livestock. J Anim Sci 73:909-918. 
177. McGorum BC, Pirie RS, Fry SC. 2012. Quantification of cyanogenic glycosides in white clover (Trifolium repens L.) from horse pastures in relation to equine grass sickness. Grass Forage Sci 67:274-279.

178. Kemp PF, Aller JY. 2004. Bacterial diversity in aquatic and other environments: What 16S rDNA libraries can tell us. FEMS Microbiol Ecol 47:161-177.

179. Bi R, Spadiut O, Lawoko M, Brumer H, Henriksson G. 2012. Isolation and identification of microorganisms from soil able to live on lignin as a carbon source and to produce enzymes which cleave the beta-O-4 bond in a lignin model compound. Cellul Chem Technol 46:227-242.

180. Riis V, Lorbeer H, Babel W. 1998. Extraction of microorganisms from soil: Evaluation of the efficiency by counting methods and activity measurements. Soil Biol Biochem 30:1573-1581.

181. Liu J, Li J, Feng L, Cao H, Cui Z. 2010. An improved method for extracting bacteria from soil for high molecular weight DNA recovery and BAC library construction. J Microbiol 48:728-733.

182. Bunk B, Schulz A, Stammen S, Münch R, Warren MJ, Rohde M, Jahn D, Biedendieck R. 2010. A short story about a big magic bug. Bioeng Bugs 1:85-91.

183. Veselá $A B$, Franc M, Pelantová H, Kubáč D, Vejvoda V, Šulc M, Bhalla TC, Macková M, Lovecká P, Janů P, Demnerová K, Martínková L. 2010. Hydrolysis of benzonitrile herbicides by soil actinobacteria and metabolite toxicity. Biodegradation 21:761-770.

184. U.S. Department of the Interior. 2016. 2016 pesticide use maps.

185. Mishra J, Prakash J, Arora NK. 2016. Role of beneficial soil microbes in sustainable agriculture and environmental management. Clim Chang Environ Sustain 4:137-149.

186. Banea-Mayambu JP, Tylleskär T, Gitebo N, Matadi N, Gebre-Medhin M, Rosling H. 1997. Geographical and seasonal association between linamarin and cyanide exposure from cassava and the upper motor neurone disease konzo in former Zaire. Trop Med Int Heal 2:1143-1151.

187. Ballhorn DJ, Kautz S, Heil M, Hegeman AD. 2009. Cyanogenesis of wild lima bean (Phaseolus lunatus L.) is an efficient direct defence in nature. PLoS One 4:e5450.

188. Shahidi F, Wanasundara PKJPD. 1997. Cyanogenic glycosides of flaxseeds., p. 171-185. In Shahidi, F (ed.), Antinutrients and phytochemicals in food, 1st ed. American Chemical Society, Washington, DC, USA.

189. Mao JC, Putterman M. 1968. Accumulation in Gram-postive and Gram-negative bacteria as a mechanism of resistance to erythromycin. J Bacteriol 95:1111-1117.

190. Lambert PA. 2002. Cellular impermeability and uptake of biocides and antibiotics in Grampositive bacteria and mycobacteria. Symp Ser Soc Appl Microbiol 92:46S-54S.

191. Gladstone BP, Cona A, Shamsrizi P, Vilken T, Kern W V., Malek N, Tacconelli E. 2017. Antimicrobial resistance rates in Gram-positive bacteria do not drive glycopeptides use. PLoS One 12:e0181358.

192. Lăzăroaie MM. 2010. Multiple responses of Gram-positive and Gram-negative bacteria to mixture of hydrocarbons. Brazilian J Microbiol 41:649-667.

193. Mai-Prochnow A, Clauson M, Hong J, Murphy AB. 2016. Gram positive and Gram negative bacteria differ in their sensitivity to cold plasma. Sci Rep 6:38610. 
194. Friedman A, Blecher K, Sanchez D, Tuckman-Vernon C, Gialanella P, Friedman JM, Martinez LR, Nosanchuk JD. 2011. Susceptibility of Gram-positive and -negative bacteria to novel nitric oxidereleasing nanoparticle technology. Virulence 2:217-221.

195. Mathias PM, Elliott JR, Klamt A. 2008. Butadiene purification using polar solvents. Analysis of solution nonideality using data and estimation methods. Ind Eng Chem Res 47:4996-5004.

196. McConvey IF, Woods D, Lewis M, Gan Q, Nancarrow P. 2012. The importance of acetonitrile in the pharmaceutical industry and opportunities for its recovery from waste. Org Process Res Dev 16:612-624.

197. Yamada Y, Furukawa K, Sodeyama K, Kikuchi K, Yaegashi M, Tateyama Y, Yamada A. 2014. Unusual stability of acetonitrile-based superconcentrated electrolytes for fast-charging lithiumion batteries. J Am Chem Soc 136:5039-5046.

198. Gaytán P. 2009. Chemical synthesis of oligonucleotides using acetone as a washing solvent. Biotechniques 47:701-702.

199. Fritz R, Ruth W, Kragl U. 2009. Assessment of acetone as an alternative to acetonitrile in peptide analysis by liquid chromatography/mass spectrometry. Rapid Commun Mass Spectrom 23:2139-2145.

200. Henderson RK, Jiménez-González C, Constable DJC, Alston SR, Inglis GGA, Fisher G, Sherwood J, Binks SP, Curzons AD. 2011. Expanding GSK's solvent selection guide - Embedding sustainability into solvent selection starting at medicinal chemistry. Green Chem 13:854-862.

201. Gilomen K, Stauffer HP, Meyer VR. 1995. Detoxification of acetonitrile - Water wastes from liquid chromatography. Chromatographia 41:488-491.

202. Youngprasert B, Poochinda K, Ngamprasertsith S. 2010. Treatment of acetonitrile by catalytic supercritical water oxidation in compact-sized reactor. J Water Resour Prot 02:222-226.

203. Micaroni RC da CM, Bueno MIMS, Jardim WDF. 2004. Degradation of acetonitrile residues using oxidation processes. J Braz Chem Soc 15:509-513.

204. Kubsad V, Gupta SK, Chaudhari S. 2011. Biodegradation of wastewater containing cyanide, acetonitrile, and acrylonitrile using RBC and shock loading study. Can J Chem Eng 89:15361544.

205. Li T, Liu J, Bai R, Wong FS. 2008. Membrane-aerated biofilm reactor for the treatment of acetonitrile wastewater. Environ Sci Technol 42:2099-2104.

206. Kohyama E, Yoshimura A, Aoshima D, Yoshida T, Kawamoto H, Nagasawa T. 2006. Convenient treatment of acetonitrile-containing wastes using the tandem combination of nitrile hydratase and amidase-producing microorganisms. Appl Microbiol Biotechnol 72:600-606.

207. Nawaz MS, Chapatwala KD, Wolfram JH. 1989. Degradation of acetonitrile by Pseudomonas putida. Appl Environ Microbiol 55:2267-2274.

208. Firmin JL, Gray DO. 1976. The biochemical pathway for the breakdown of methyl cyanide (acetonitrile) in bacteria. Bioche J 158:223-229.

209. Danana Goud H, Parekh L, Ramakrishnan C V. 1985. Bacterial profile of petrochemical industry effluents. Environ Pollution Ser A, Ecol Biol 39:27-37. 
210. Acharya A, Desai AJ. 1997. Studies on utilization of acetonitrile by Rhodococcus erythropolis A10. World J Microbiol Biotechnol 13:175-178.

211. Okamoto S, Eltis LD. 2007. Purification and characterization of a novel nitrile hydratase from Rhodococcus sp. RHA1. Mol Microbiol 65:828-838.

212. Miller JM, Gray DO. 1982. The utilization of nitriles and amides by a Rhodococcus species. Microbiology 128:1803-1809.

213. Martínková L, Uhnáková B, Pátek M, Nesvera J, Kren V. 2009. Biodegradation potential of the genus Rhodococcus. Environ Int 35:162-177.

214. Sun SL, Yang WL, Fang WW, Zhao YX, Guo L, Dai YJ. 2018. The plant growth-promoting rhizobacterium Variovorax boronicumulans CGMCC 4969 regulates the level of indole-3-acetic acid synthesized from indole-3-acetonitrile. Appl Environ Microbiol 84:e00298-18.

215. Zhang H-J, Zhou Q-W, Zhou G-C, Cao Y-M, Dai Y-J, Ji W-W, Shang G-D, Yuan S. 2012. Biotransformation of the neonicotinoid insecticide thiacloprid by the bacterium Variovorax boronicumulans strain $\mathrm{J} 1$ and mediation of the major metabolic pathway by nitrile hydratase. $\mathrm{J}$ Agric Food Chem 60:153-159.

216. Kato Y, Ryoko O, Asano Y. 2000. Distribution of aldoxime dehydratase in microorganisms. Appl Environ Microbiol 66:2290-2296.

217. Xue Y-P, Yang Y-K, Lv S-Z, Liu Z-Q, Zheng Y-G. 2016. High-throughput screening methods for nitrilases. Appl Microbiol Biotechnol 100:3421-3432.

218. Liu Z-Q, Zhang X-H, Xue Y-P, Xu M, Zheng Y-G. 2014. Improvement of Alcaligenes faecalis nitrilase by gene site saturation mutagenesis and its application in stereospecific biosynthesis of ( R )-(-)-mandelic acid. J Agric Food Chem 62:4685-4694.

219. Banerjee A, Kaul P, Sharma R, Banerjee UC. 2003. A high-throughput amenable colorimetric assay for enantioselective screening of nitrilase-producing microorganisms using $\mathrm{pH}$ sensitive indicators. J Biomol Screen 8:559-565.

220. Hu J-G, Wang Y-J, Zheng Y-G, Shen Y-C. 2007. Isolation of glycolonitrile-hydrolyzing microorganism based on colorimetric reaction. Enzyme Microb Technol 41:244-249.

221. Zhu Q, Fan A, Wang Y, Zhu X, Wang Z, Wu M, Zheng Y. 2007. Novel sensitive high-throughput screening strategy for nitrilase-producing strains. Appl Environ Microbiol 73:6053-6057.

222. Yazbeck DR, Durao PJ, Xie Z, Tao J. 2006. A metal ion-based method for the screening of nitrilases. J Mol Catal B Enzym 39:156-159.

223. Kiianitsa K, Solinger JA, Heyer WD. 2003. NADH-coupled microplate photometric assay for kinetic studies of ATP-hydrolyzing enzymes with low and high specific activities. Anal Biochem 321:266-271.

224. Avigad G. 1978. An NADH coupled assay system for galactose oxidase. Anal Biochem 86:470476.

225. Wolfson NA, Pitcairn CA, Sullivan ED, Joseph CG, Fierke CA. 2014. An enzyme-coupled assay measuring acetate production for profiling histone deacetylase specificity. Anal Biochem 456:61-69. 
226. Kaltwasser H, Schlegel HG. 1966. NADH-dependent coupled enzyme assay for urease and other ammonia-producing systems. Anal Biochem 16:132-138.

227. Takahashi-Íñiguez T, Aburto-Rodríguez N, Vilchis-González AL, Flores ME. 2016. Function, kinetic properties, crystallization, and regulation of microbial malate dehydrogenase. J Zhejiang Univ B 17:247-261.

228. Veselá AB, Rucká L, Kaplan O, Pelantová H, Nešvera J, Pátek M, Martínková L. 2016. Bringing nitrilase sequences from databases to life: The search for novel substrate specificities with a focus on dinitriles. Appl Microbiol Biotechnol 100:2193-2202.

229. Nagasawa T, Mauger J, Yamada H. 1990. A novel nitrilase, arylacetonitrilase, of Alcaligenes faecalis JM3. Purification and characterization. Eur J Biochem 194:765-772.

230. Yamamoto K, Fujimatsu I, Komatsu K-I. 1992. Purification and characterization of the nitrilase from Alcaligenes faecalis ATCC 8750 responsible for enantioselective hydrolysis of mandelonitrile. J Ferment Bioeng 73:425-430.

231. Liu ZQ, Dong LZ, Cheng F, Xue YP, Wang YS, Ding JN, Zheng YG, Shen YC. 2011. Gene cloning, expression, and characterization of a nitrilase from Alcaligenes faecalis ZJUTB10. J Agric Food Chem 59:11560-11570.

232. Bhatia SK, Mehta PK, Bhatia RK, Bhalla TC. 2014. Purification and characterization of arylacetonitrile-specific nitrilase of Alcaligenes sp. MTCC 10675. Biotechnol Appl Biochem 61:459-465.

233. Zhu D, Mukherjee C, Biehl ER, Hua L. 2007. Discovery of a mandelonitrile hydrolase from Bradyrhizobium japonicum USDA110 by rational genome mining. J Biotechnol 129:645-650.

234. Sun H, Gao W, Fan H, Wang H, Wei D. 2015. Cloning, purification and evaluation of the enzymatic properties of a novel arylacetonitrilase from Luminiphilus syltensis NOR5-1B: A potential biocatalyst for the synthesis of mandelic acid and its derivatives. Biotechnol Lett 37:1655-1661.

235. Kiziak C, Conradt D, Stolz A, Mattes R, Klein J. 2005. Nitrilase from Pseudomonas fluorescens EBC191: Cloning and heterologous expression of the gene and biochemical characterization of the recombinant enzyme. Microbiology 151:3639-3648.

236. Kiziak C, Klein J, Stolz A. 2007. Influence of different carboxy-terminal mutations on the substrate-, reaction- and enantiospecificity of the arylacetonitrilase from Pseudomonas fluorescens EBC191. Protein Eng Des Sel 20:385-396.

237. Kiziak C, Stolz A. 2009. Identification of amino acid residues responsible for the enantioselectivity and amide formation capacity of the arylacetonitrilase from Pseudomonas fluorescens EBC191. Appl Environ Microbiol 75:5592-5599.

238. Sosedov O, Baum S, Bürger S, Matzer K, Kiziak C, Stolz A. 2010. Construction and application of variants of the Pseudomonas fluorescens EBC191 arylacetonitrilase for increased production of acids or amides. Appl Environ Microbiol 76:3668-3674.

239. Sosedov O, Stolz A. 2014. Random mutagenesis of the arylacetonitrilase from Pseudomonas fluorescens EBC191 and identification of variants, which form increased amounts of mandeloamide from mandelonitrile. Appl Microbiol Biotechnol 98:1595-1607. 
240. Sosedov O, Stolz A. 2015. Improvement of the amides forming capacity of the arylacetonitrilase from Pseudomonas fluorescens EBC191 by site-directed mutagenesis. Appl Microbiol Biotechnol 99:2623-2635.

241. Xue YP, Jiao B, Hua DE, Cheng F, Liu ZQ, Zheng YG. 2017. Improving catalytic performance of an arylacetonitrilase by semirational engineering. Bioprocess Biosyst Eng 40:1565-1572.

242. Whelan S, Goldman N. 2001. A general empirical model of protein evolution derived from multiple protein families using a maximum-likelihood approach. Mol Biol Evol 18:691-699.

243. Thykaer J, Christensen B, Nielsen J. 2002. Metabolic network analysis of an adipoyl-7-ADCAproducing strain of Penicillium chrysogenum: Elucidation of adipate degradation. Metab Eng 4:151-158.

244. Casamassimi A, Federico A, Rienzo M, Esposito S, Ciccodicola A. 2017. Transcriptome profiling in human diseases: New advances and perspectives. Int J Mol Sci 18:1652.

245. Sanders AR, Göring HHH, Duan J, Drigalenko El, Moy W, Freda J, He D, Shi J, Gejman P V. 2013. Transcriptome study of differential expression in schizophrenia. Hum Mol Genet 22:5001-5014.

246. Udhane SS, Legeza B, Marti N, Hertig D, DIserens G, Nuoffer JM, Vermathen P, Flück CE. 2017. Combined transcriptome and metabolome analyses of metformin effects reveal novel links between metabolic networks in steroidogenic systems. Sci Rep 7:8652.

247. Tewari SG, Prigge ST, Reifman J, Wallqvist A. 2017. Using a genome-scale metabolic network model to elucidate the mechanism of chloroquine action in Plasmodium falciparum. Int $\mathrm{J}$ Parasitol Drugs Drug Resist 7:138-146.

248. Jensen PA, Zhu Z, van Opijnen T. 2017. Antibiotics disrupt coordination between transcriptional and phenotypic stress responses in pathogenic bacteria. Cell Rep 20:1705-1716.

249. Qin H, Lo NW-S, Loo JF-C, Lin X, Yim AK-Y, Tsui SK-W, Lau TC-K, Ip M, Chan T-F. 2018. Comparative transcriptomics of multidrug-resistant Acinetobacter baumannii in response to antibiotic treatments. Sci Rep 8:3515.

250. Schwartz T, Armant O, Bretschneider N, Hahn A, Kirchen S, Seifert M, Dötsch A. 2015. Whole genome and transcriptome analyses of environmental antibiotic sensitive and multi-resistant Pseudomonas aeruginosa isolates exposed to waste water. and tap water. Microb Biotechnol 8:116-130.

251. Khaledi A, Schniederjans M, Pohl S, Rainer R, Bodenhofer U, Xia B, Klawonn F, Bruchmann S, Preusse M, Eckweiler D, Dötsch A, Häussler S. 2016. Transcriptome profiling of antimicrobial resistance in Pseudomonas aeruginosa. Antimicrob Agents Chemother 60:4722-4733.

252. Wecke T, Mascher T. 2011. Antibiotic research in the age of omics: From expression profiles to interspecies communication. J Antimicrob Chemother 66:2689-2704.

253. Dong Y, Hu J, Fan L, Chen Q. 2017. RNA-seq-based transcriptomic and metabolomic analysis reveal stress responses and programmed cell death induced by acetic acid in Saccharomyces cerevisiae. Sci Rep 7:42659.

254. Anfelt J, Hallström B, Nielsen J, Uhlén M, Hudson EP. 2013. Using transcriptomics to improve butanol tolerance of Synechocystis sp. strain PCC 6803. Appl Environ Microbiol 79:7419-7427. 
255. Frusciante S, Diretto G, Bruno M, Ferrante P, Pietrella M, Prado-Cabrero A, Rubio-Moraga A, Beyer P, Gomez-Gomez L, Al-Babili S, Giuliano G. 2014. Novel carotenoid cleavage dioxygenase catalyzes the first dedicated step in saffron crocin biosynthesis. Proc Natl Acad Sci 111:1224612251.

256. Dillies MA, Rau A, Aubert J, Hennequet-Antier C, Jeanmougin M, Servant N, Keime C, Marot NS, Castel D, Estelle J, Guernec G, Jagla B, Jouneau L, Laloë D, Le Gall C, Schaëffer B, Le Crom S, Guedj M, Jaffrézic F. 2013. A comprehensive evaluation of normalization methods for Illumina high-throughput RNA sequencing data analysis. Brief Bioinform 14:671-683.

257. Lin Y, Golovnina K, Chen Z-X, Lee HN, Negron YLS, Sultana H, Oliver B, Harbison ST. 2016. Comparison of normalization and differential expression analyses using RNA-Seq data from 726 individual Drosophila melanogaster. BMC Genomics 17:28.

258. Zyprych-Walczak J, Szabelska A, Handschuh L, Górczak K, Klamecka K, Figlerowicz M, Siatkowski I. 2015. The impact of normalization methods on RNA-seq data analysis. Biomed Res Int 2015:621690.

259. Han H, Men K. 2018. How does normalization impact RNA-seq disease diagnosis? J Biomed Inform 85:80-92.

260. Robinson MD, Oshlack A. 2010. A scaling normalization method for differential expression analysis of RNA-seq data. Genome Biol 11:R25.

261. Robinson MD, McCarthy DJ, Smyth GK. 2010. edgeR: A Bioconductor package for differential expression analysis of digital gene expression data. Bioinformatics 26:139-140.

262. Love MI, Huber W, Anders S. 2014. Moderated estimation of fold change and dispersion for RNA-seq data with DESeq2. Genome Biol 15:550.

263. Kim HB, Borewicz K, White BA, Singer RS, Sreevatsan S, Tu ZJ, Isaacson RE. 2012. Microbial shifts in the swine distal gut in response to the treatment with antimicrobial growth promoter, tylosin. Proc Natl Acad Sci 109:15485-15490.

264. Svensson K, Paruch L, Gaby JC, Linjordet R. 2018. Feeding frequency influences process performance and microbial community composition in anaerobic digesters treating steam exploded food waste. Bioresour Technol 269:276-284.

265. Calicioglu O, Shreve MJ, Richard TL, Brennan RA. 2018. Effect of $\mathrm{pH}$ and temperature on microbial community structure and carboxylic acid yield during the acidogenic digestion of duckweed. Biotechnol Biofuels 11:275.

266. Klingenberg H, Meinicke P. 2017. How to normalize metatranscriptomic count data for differential expression analysis. PeerJ 5:e3859.

267. McNulty NP, Wu M, Erickson AR, Pan C, Erickson BK, Martens EC, Pudlo NA, Muegge BD, Henrissat B, Hettich RL, Gordon Jl. 2013. Effects of diet on resource utilization by a model human gut microbiota containing bacteroides cellulosilyticus WH2, a symbiont with an extensive glycobiome. PLoS Biol 11:e1001637.

268. Nacke H, Fischer C, Thürmer A, Meinicke P, Daniel R. 2014. Land use type significantly affects microbial gene transcription in soil. Microb Ecol 67:919-930. 
269. Jorth P, Turner KH, Gumus P, Nizam N, Buduneli N, Whiteley M. 2014. Metatranscriptomics of the human oral microbiome during health and disease. MBio 5:e01012-14.

270. Jiang Y, Xiong X, Danska J, Parkinson J. 2016. Metatranscriptomic analysis of diverse microbial communities reveals core metabolic pathways and microbiome-specific functionality. Microbiome 4:2.

271. De Filippis F, Genovese A, Ferranti P, Gilbert JA, Ercolini D. 2016. Metatranscriptomics reveals temperature-driven functional changes in microbiome impacting cheese maturation rate. Sci Rep 6:21871.

272. Men Y, Yu K, Bælum J, Gao Y, Tremblay J, Prestat E, Stenuit B, Tringe SG, Jansson J, Zhang T, Alvarez-Cohen L. 2017. Metagenomic and metatranscriptomic analyses reveal the structure and dynamics of a dechlorinating community containing Dehalococcoides mccartyi and corrinoidproviding microorganisms under cobalamin-limited conditions. Appl Environ Microbiol 83:e03508-16.

273. Peng J, Wegner CE, Bei Q, Liu P, Liesack W. 2018. Metatranscriptomics reveals a differential temperature effect on the structural and functional organization of the anaerobic food web in rice field soil. Microbiome 6:169.

274. Seth EC, Taga ME. 2014. Nutrient cross-feeding in the microbial world. Front Microbiol 5:350.

275. Muller EEL, Faust K, Widder S, Herold M, Martínez Arbas S, Wilmes P. 2018. Using metabolic networks to resolve ecological properties of microbiomes. Curr Opin Syst Biol 8:73-80.

276. Lawson CE, Wu S, Bhattacharjee AS, Hamilton JJ, McMahon KD, Goel R, Noguera DR. 2017. Metabolic network analysis reveals microbial community interactions in anammox granules. Nat Commun 8:15416.

277. D'Souza G, Shitut S, Preussger D, Yousif G, Waschina S, Kost C. 2018. Ecology and evolution of metabolic cross-feeding interactions in bacteria. Nat Prod Rep 35:455-488. 
Chapter VI

Appendix 


\section{Appendix}

\subsection{Publications}

Egelkamp, R., Schneider, D., Hertel, R. \& Daniel, R. (2017). Nitrile-degrading bacteria isolated from compost. Front. Environ. Sci., 5: 56. https://doi.org/10.3389/fenvs.2017.00056

Dormeyer, M.*, Egelkamp, R.*, Thiele, MJ., Hammer, E., Gunka, K., Stannek, L., Völker, U. \& Commichau, FM. (2015). A novel engineering tool in the Bacillus subtilis toolbox: inducer-free activation of gene expression by selection-driven promoter decryptification. Microbiology 161: 354361. https://doi.org/10.1099/mic.0.000001

*Shared first author

Stannek, L., Egelkamp, R., Gunka, K. \& Commichau, FM. (2014). Monitoring intraspecies competition in a bacterial cell population by cocultivation of fluorescently labelled strains. J. Vis. Exp. 83: e51196. https://doi.org/10.3791/51196 


\subsection{Posters at conferences}

Friedrich, I., Egelkamp, R., Hertel, R. \& Daniel, R. (2017). Characterization of nitrilases isolated from enrichment cultures. $7^{\text {th }}$ European Conference on Prokaryotic and Fungal Genomics (ProkaGENOMICS), Göttingen (19.09.2017 - 22.09.2017).

Egelkamp, R., Zimmermann, T., Hertel, R. \& Daniel, R. (2017). Influence of nitriles on bacterial communities. Microbiology and Infection $2017-5^{\text {th }}$ Joint Conference of the DGHM \& VAAM, Würzburg (05.03.2017-08.03.2017).

Egelkamp, R., Hertel, R., Brady, S. \& Daniel, R. (2015). Novel approach for identification of biocatalysts by reverse omics techniques. $6^{\text {th }}$ European Conference on Prokaryotic and Fungal Genomics (ProkaGENOMICS), Göttingen (29.09.2015-02.10.2015). 


\subsection{Declaration of independent work}

I, Richard Egelkamp, hereby declare that the doctoral thesis

\section{"Novel approach for identification of biocatalysts by reverse omics techniques"}

has been written independently and with no other sources and aids than quoted. Moreover, I have not used unauthorized assistance and have not submitted this thesis previously in any form for another degree at any institution or university.

Richard Egelkamp

Göttingen, 21.12.2018 


\subsection{Acknowledgment}

Every research project has its ups and downs and a PhD thesis can feel like an emotional roller coaster. On good days, the Nobel Price seems within reach, while on bad days, the project feels like an impossible task. This thesis would not have been written without the continuous support and believe of many people and I wanted to save the last page for them.

First, I want to thank Prof. Dr. Rolf Daniel. During one of the hardest times of my life, you gave me the opportunity to start with this project. It was a challenging task, but also one of the most interesting topics I could imagine. Without your constant support, your ideas, and your corrections, none of this would have been possible.

I am also very grateful to PD Dr. Michael Hoppert for being my co-examiner. You followed my topic with interest and had constructive ideas whenever needed.

I would also like to thank Prof. Dr. Jörg Stülke. A few years ago, you taught me the basics of lab work and since then you always had a sympathetic ear for me. Additional thanks go to Prof. Dr. Gerhard Braus, Prof. Dr. Kai Heimel, and Prof. Dr. Stefanie Pöggeler for being part of my examination board.

Special thanks to my supervisors Silja and Robert. Both of you had great impact on this project and shaped my idea of science. Especially the many lessons I have learned during discussions, brain stormings, and private conversations with Robert will accompany me for the rest of my life.

I would also like to thank my students Till, Ankita, and Ines. Without your passionate work and the obtained results, several pages of this thesis would be blank. It has been a pleasure with all of you and I whish you all the best for your future careers.

Thanks also to the whole department: Whenever help was needed, I could be sure to find it. Whether for sequencing (Anja, Mechthild, and Melanie), for (bio)informatics (Dominik, Marc, Sascha, and Tarek), or for daily organization (Daniela, Nicole, and Petra), there were always the right people at hand. A special thank also to Birgit and Jacqueline as your (emotional) support made the last phase more bearable. Further, I want to thank Amélie, Avril, Dirk, Genis, Ines (again!), Katja, Kristin, Simone, Randi, and Tatiana for being more than just colleagues. This project might have been finished months earlier without all the distraction, but I would never exchange all the fun and laughter we had for it.

Of course, I also want to thank my family. Only your support and belief in me made this work possible. I am pretty sure my science talk has gotten on your nerves quite often, but you never complained. And finally, my biggest thanks go to my girlfriend Katrina. You have motivated me all the time and stayed at my side even during my worst moods. I can be myself around you, and that is why I dedicate this work to you. 